\title{
Improvements to CTF Closure Models for Modeling of Two-Phase Flow
}

Approved for public release. Distribution is unlimited.

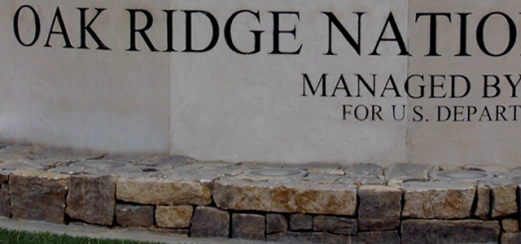

R. Salko

V. Kumar

B. Hizoum

W. Gurecky

July 31, 2020 


\section{DOCUMENT AVAILABILITY}

Reports produced after January 1, 1996, are generally available free via US Department of Energy (DOE) SciTech Connect.

Website: www.osti.gov/

Reports produced before January 1, 1996, may be purchased by members of the public from the following source:

National Technical Information Service

5285 Port Royal Road

Springfield, VA 22161

Telephone: 703-605-6000 (1-800-553-6847)

TDD: $703-487-4639$

Fax: 703-605-6900

E-mail: info@ntis.gov

Website: http://classic.ntis.gov/

Reports are available to DOE employees, DOE contractors, Energy Technology Data Exchange representatives, and International Nuclear Information System representatives from the following source:

Office of Scientific and Technical Information

PO Box 62

Oak Ridge, TN 37831

Telephone: 865-576-8401

Fax: 865-576-5728

E-mail: report@osti.gov

Website: http://www.osti.gov/contact.html

This report was prepared as an account of work sponsored by an agency of the United States Government. Neither the United States Government nor any agency thereof, nor any of their employees, makes any warranty, express or implied, or assumes any legal liability or responsibility for the accuracy, completeness, or usefulness of any information, apparatus, product, or process disclosed, or represents that its use would not infringe privately owned rights. Reference herein to any specific commercial product, process, or service by trade name, trademark, manufacturer, or otherwise, does not necessarily constitute or imply its endorsement, recommendation, or favoring by the United States Government or any agency thereof. The views and opinions of authors expressed herein do not necessarily state or reflect those of the United States Government or any agency thereof. 
Reactor Nuclear Systems Division

\title{
Improvements to CTF Closure Models for Modeling of Two-Phase Flow
}

\author{
Authors \\ R. Salko \\ V. Kumar \\ B. Hizoum \\ W. Gurecky
}

Date Published: July 31, 2020

\author{
Prepared by \\ OAK RIDGE NATIONAL LABORATORY \\ Oak Ridge, TN 37831-6283 \\ managed by \\ UT-Battelle, LLC \\ for the \\ US DEPARTMENT OF ENERGY \\ under contract DE-AC05-00OR22725
}





\section{CONTENTS}

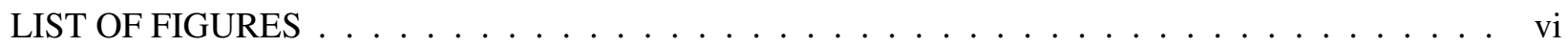

LIST OF TABLES $\ldots \ldots \ldots \ldots \ldots \ldots \ldots \ldots \ldots \ldots$ vii

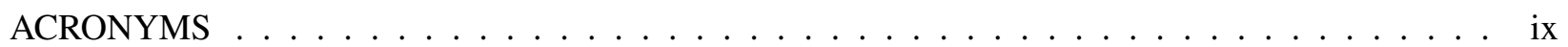

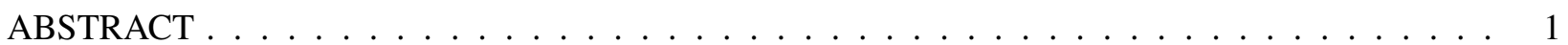

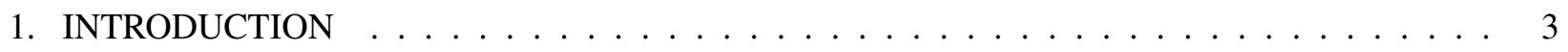

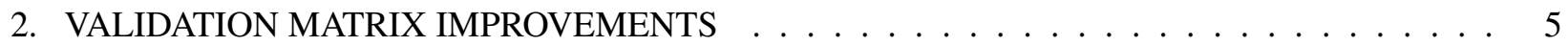

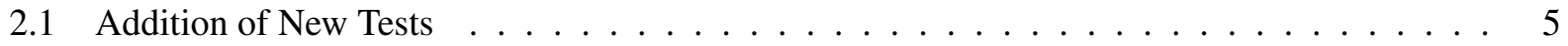

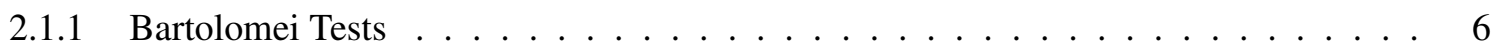

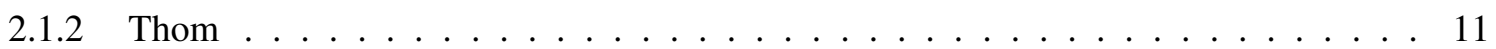

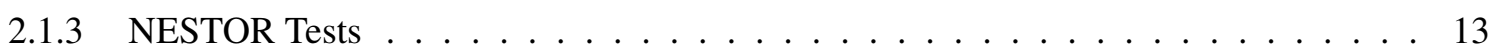

2.2 Validation Matrix Driver . . . . . . . . . . . . . . . . . 13

3. SUBCOOLED BOILING MODEL IMPROVEMENTS . . . . . . . . . . . . . . . . 17

3.1 Basu ONB criteria . . . . . . . . . . . . . . . . . . . . . . . . 17

3.2 Gorenflo Heat Transfer Model . . . . . . . . . . . . . . . . . . . . . . . . 18

3.3 Bubble Detachment Criteria . . . . . . . . . . . . . . . . . . . . . 19

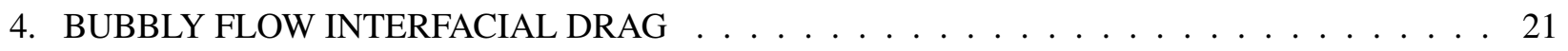

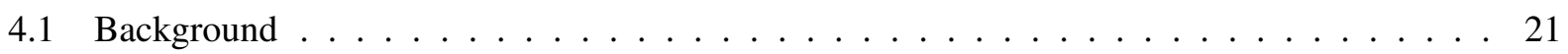

4.2 Drift Flux Model . . . . . . . . . . . . . . . . . . . . . . . . 21

4.3 Interfacial Drag Model Form . . . . . . . . . . . . . . . . . . . 23

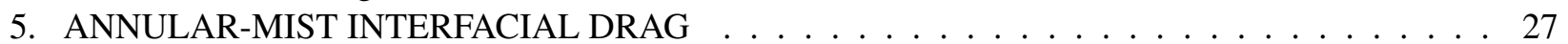

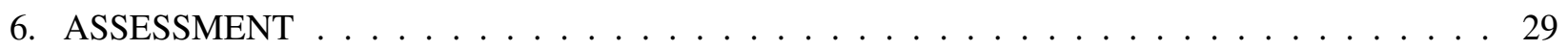

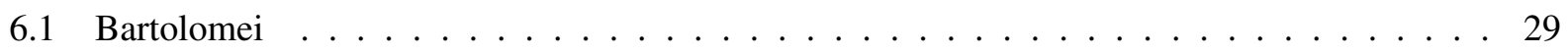

6.2 PSBT Single Channel . . . . . . . . . . . . . . . . . . . . . 31

6.3 PSBT Bundle Tests . . . . . . . . . . . . . . . . . . . . . 33

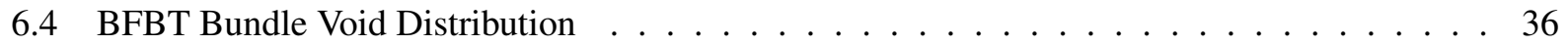

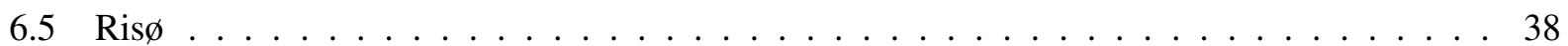

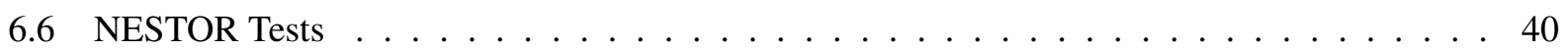

6.7 Rohsenow Tests . . . . . . . . . . . . . . . . . . . . . . 43

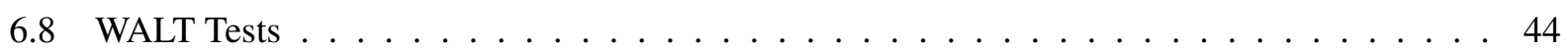

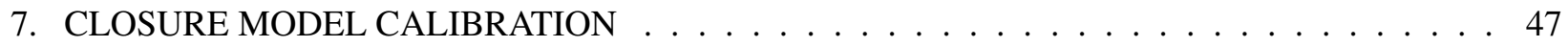

7.1 Bayesian Calibration Setup . . . . . . . . . . . . . . . . . . 47

7.1 .1 MCMC Implementation . . . . . . . . . . . . . . . . . . . . 48

7.2 Calibration Parameter Selection and Development of Surrogate Models . . . . . . . . . 49

7.2 .1 CTF Models . . . . . . . . . . . . . . . . . . . 53

7.2 .2 Gradient Boosting . . . . . . . . . . . . . . . . . . 53

7.2.3 Gradient Boosting Model Construction Results . . . . . . . . . . . . . . . . 54

7.3 Calibration Results . . . . . . . . . . . . . . . . . . . 55

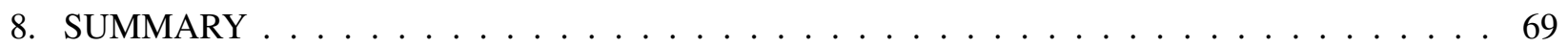

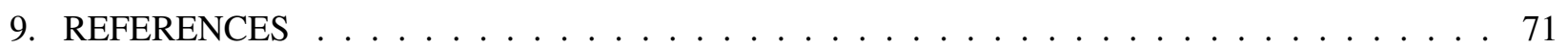





\section{LIST OF FIGURES}

1 Comparison of CTF and experimental data of wall temperature, mean liquid temperature, and void fraction for Case $1 . \ldots \ldots \ldots \ldots \ldots$

2 Overall comparison of CTF vs. experimental void fractions by using the subcooled

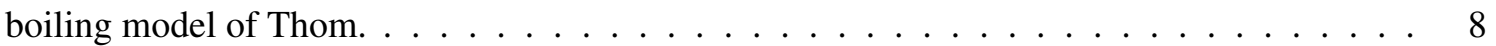

3 Comparison of CTF and experimental data for variation in mass flux at 70 bar and a heat flux of $0.8 \mathrm{MW} / \mathrm{m}^{2} \ldots \ldots \ldots \ldots \ldots \ldots$

4 Comparison of CTF and experimental data for variation in mass flux at 70 bar and a mass

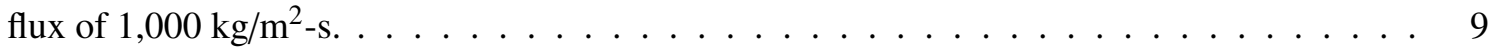

5 Comparison of CTF and experimental data for variation in mass flux at 150 bar and a mass flux of $2,000 \mathrm{~kg} / \mathrm{m}^{2}-\mathrm{s} \ldots \ldots \ldots \ldots \ldots \ldots \ldots$

6 Comparison of CTF and experimental data for variation in system pressure at a mass flux of $2,000 \mathrm{~kg} / \mathrm{m}^{2}$-s and a heat flux of $1.1 \mathrm{MW} / \mathrm{m}^{2} \ldots \ldots \ldots \ldots \ldots$

7 Comparison of predicted and experimental data from Thom for the temperature difference between the outer wall and the bulk liquid vs. liquid subcooling. The " $\mathrm{x}$ " symbols are CTF predictions and the circles are experimental data. . . . . . . . . . . . . 12

8 Comparison of predicted vs. experimental data from Thom for the temperature difference between the outer wall and the bulk liquid. . . . . . . . . . . . . . . . . . . . 12

9 Geometry of the heated NESTOR tests. . . . . . . . . . . . . . . . . . . . . . 14

10 CTF prediction of two-phase pressure drop in the Ris $\varnothing$ facility by using (a) the base model, (b) the unstable film via the Wallis model for interfacial drag, and (c) the Lane model. 28

11 CTF prediction of void fraction in Bartolomei cases by using original models, drift-flux, and drift-flux/ONB models. . . . . . . . . . . . . . . . . . . 30

12 Validation of CTF to PSBT single-channel tests (Series 1-4) by using baseline models, drift-flux models, and ONB models. . . . . . . . . . . . . . . . . . 32

13 Comparison of CTF and VIPRE-01 mean error for PSBT single-channel tests. CTF results include the baseline models, drift-flux model, and drift-flux combined with ONB

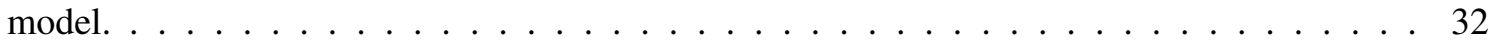

14 PSBT bundle test results using CTF baseline, drift-flux, and drift-flux/ONB models. . . . . . 34

15 Benchmark of CTF and VIPRE-01 for PSBT bundle tests using drift-flux and ONB model in CTF. . . . . . . . . . . . . . . . . . . . . 35

16 Comparison of CTF vs. experimental area-weighted bundle-averaged void fractions at outlet for the BFBT tests by using original, drift-flux, and drift-flux/ONB models. . . . . . . 37

17 CTF prediction of two-phase pressure drop in Risø facility using the drift-flux model and the drift-flux model with tube distribution parameter and ONB model. . . . . . . . . . . . 39

18 Comparison of spread of error between CTF predictions and NESTOR experimental results for surface temperature in Span 1a of the heated test facility when using the CTF Thom model vs. the new ONB model. . . . . . . . . . . . . . . . . . . . .

19 Comparison of spread of error between CTF predictions and NESTOR experimental results for surface temperature in Span $1 \mathrm{~b}$ of the heated test facility when using the CTF Thom model vs. the new ONB model. . . . . . . . . . . . . . . . . . . . . . 41

20 Comparison of spread of error between CTF predictions and NESTOR experimental results for surface temperature in Span 2a of the heated test facility when using the CTF Thom model vs. the new ONB model. 
21 Comparison of spread of error between CTF predictions and NESTOR experimental results for surface temperature in Span $2 \mathrm{~b}$ of the heated test facility when using the CTF Thom model vs the new ONB model. . . . . . . . . . . . . . . . . . . . . . 42

22 Difference between CTF and experimental wall temperatures for the Rohsenow experiments. 44

23 Difference between CTF and experimental wall temperatures vs. heater flux for the

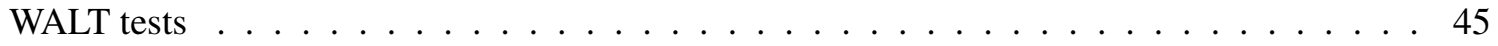

24 CTF sensitivity analysis in CTF void fraction prediction for the boiling heat transfer

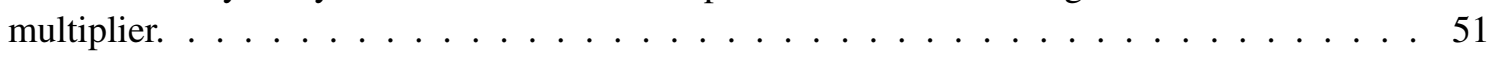

25 CTF sensitivity analysis in CTF void fraction prediction for the interfacial drag small

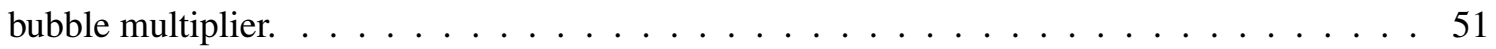

26 Change in the training and testing RMSE as a function of the number of trees for the gradient boosted surrogate model for the PSBT single-channel tests. . . . . . . . . . . 56

27 Surrogate model for PSBT single-subchannel tests (void fraction) . . . . . . . . . . . . . 57

28 Surrogate model for BFBT void distribution tests (void fraction) . . . . . . . . . . . . 57

29 Surrogate model for Ris $\varnothing 200$ series (adiabatic) tests (two-phase pressure gradient). . . . . . 58

30 Change in the distribution of calibration multipliers for different chains as a function of sample size per chain. . . . . . . . . . . . . . . . . . . 60

31 Final posterior distributions (pair-wise and marginal) of the calibration multipliers. . . . . . 61

32 Comparison of PSBT subchannel void fraction prediction mean error for different codes. . . 62

33 Comparison of PSBT series 5 bundle void fraction prediction with the calibrated model. . . . 63

34 Comparison of PSBT series 6 bundle void fraction prediction with the calibrated model. . . . 63

35 Comparison of PSBT series 7 bundle void fraction prediction with the calibrated model. . . . 64

36 Comparison of BFBT subchannel void fraction prediction with the calibrated model. . . . . 64

37 Comparison of BFBT bundle average outlet void fraction prediction with the calibrated model. . . . . . . . . . . . . . . . . . . . . . . . 65

38 Comparison of Bartolomei void fraction prediction with the calibrated model. . . . . . . . 65

39 Comparison of Ris $\varnothing 200$ series two-phase pressure-drop prediction with the calibrated model. 66

40 Comparison of Rohsenow tests wall temperature prediction with the calibrated model. . . . . 66

41 Comparison of WALT tests wall temperature prediction with the calibrated model. . . . . . . 67

42 Comparison of Thom tests delta wall temperature prediction with the calibrated model. . . . 67 


\section{LIST OF TABLES}

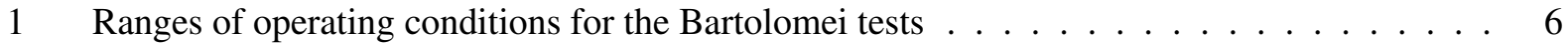

2 Ranges of operating conditions for the Thom tests. . . . . . . . . . . . . . . 11

3 Chosen calibration parameters. . . . . . . . . . . . . . . . . 50

4 Sensitivity analysis of CTF RMSE (percent void fraction) values for the PSBT single-channel tests for the selected multipliers . . . . . . . . . . . . . . 52

5 Sensitivity analysis of CTF RMSE (percent void fraction) values for the BFBT (all assembly types) channel tests for the selected multipliers. . . . . . . . . . . . . . 52

6 Sensitivity analysis of CTF RMSE (two-phase pressure gradient) values for the Ris $\emptyset$ tests for the selected multipliers. . . . . . . . . . . . . . . . . . . . 52

7 Hyperparameters for the gradient boosting model . . . . . . . . . . . . . . . 54

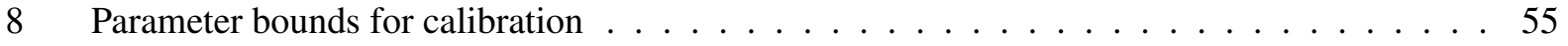

9 Summary of calibration quantile and standard deviation values. Optimal values taken as the median $(\mathrm{q} 50)$ values. . . . . . . . . . . . . . . . . . . . . 59 



\section{ACRONYMS}

ANS American Nuclear Society

BFBT BWR Full Bundle Tests

BWR boiling water reactor

CASL Consortium for Advanced Simulation of Light Water Reactors

CHF critical heat flux

DE-MCMC Differential evolution based Markov chain Monte Carlo

DREAM Differential Evolution Adaptive Metropolis

HPC high-performance computing

LWR light water reactor

MCMC Markov-chain Monte Carlo

NESTOR New Experimental Studies of Thermal Hydraulics of Rod Bundles

ONB onset of nucleate boiling

PSBT PWR Subchannel and Bundle Tests

PWR pressurized water reactor

RMSE root-mean-square error

$\mathbf{T} / \mathbf{H}$ thermal-hydraulics

VERA Virtual Environment for Reactor Applications

WALT Westinghouse Advanced Loop Tester 



\begin{abstract}
This report documents the efforts to improve the CTF prediction of void fraction and two-phase pressure drop by improving two-phase closure models. Previous validation activities have revealed that CTF tends to overpredict void fraction and two-phase pressure drop. In response to this, two approaches were taken to improve CTF's predictive capabilities. Based on findings that the interfacial drag and subcooled boiling models significantly impact void prediction, alternative closure models for these physical effects were found and implemented into the code. An extensive assessment was performed by using the existing and newly added two-phase experimental data, which showed that void prediction, wall temperature, and two-phase pressure-drop results are improved by using the newly implemented models. A second approach for improving CTF modeling accuracy involved the use of a Bayesian calibration process that uses CTF validation data to optimize selected modeling coefficients and closure model multipliers to achieve a more accurate prediction of experimental results. A similar assessment was performed with calibrated models that improved the void and pressure-drop prediction for test cases that were both included and not included in the calibration dataset. Results of this study will be used to change the models used in CTF to achieve more accurate BWR analyses moving forward. This study also identified opportunities for improving additional closure models and other opportunities to use calibration techniques to improve CTF.
\end{abstract}





\section{INTRODUCTION}

The Consortium for Advanced Simulation of Light Water Reactors (CASL)-developed Virtual Environment for Reactor Applications (VERA) core simulator is undergoing further development to support boiling water reactor (BWR) modeling. CTF is the thermal-hydraulic component of VERA that is used for thermal feedback and the prediction of thermal-hydraulic parameters of interest. CTF [34] is a thermal-hydraulic code originally developed to analyze light water reactor (LWR) geometry and operating conditions. It uses the subchannel approach to mesh the model and the two-fluid model to model two-phase flow. A third field is included that models droplet motion independently of the liquid film. With these two-phase flow modeling capabilities, CTF is applicable for BWR analysis; however, work is needed to assess the code and improve its usability for these applications.

Work done in CASL has resulted in the establishment of an initial validation matrix, which includes several two-phase experiments. This work has resulted in findings that CTF tends to overpredict void fraction and two-phase pressure drop [32] [45] [44]. Work done by Porter [27] has revealed a sensitivity of void prediction in the BWR full bundle tests (BFBT) to the interfacial drag closure models. Another study performed in Zhao et al. [45] expanded the CTF two-phase validation matrix to include the single-channel PWR subchannel and bundle tests (PSBT) and proposed that the subcooled boiling model used in CTF, which predicts vapor generation at the heater surface, also significantly impacts the quality of void predictions for two-phase models that are highly subcooled. For these reasons, this milestone was intended to primarily focus on the interfacial drag models in CTF and the subcooled boiling model.

First, the validation matrix was expanded and improved by adding more two-phase tests related to void and wall heat transfer prediction. A validation driver was also developed to allow for the rapid running and reassessment of CTF results. This work is documented in Section 2.

One milestone goal was to investigate the use of data calibration techniques and two-phase experimental data to discover and correct implicit biases in the CTF closure models. Before doing this, the targeted closure models were reviewed and the literature was searched for common modeling practices in other thermal-hydraulics $(\mathrm{T} / \mathrm{H})$ codes and other available models that could be tested in CTF. The goal was to replace existing models with more physically correct, accurate models to limit the amount of calibration needed and provide a better model form for calibration. New models were identified for both interfacial drag and subcooled boiling, which were implemented into CTF and tested. An onset of nucleate boiling (ONB) model was implemented and tested, which is documented in Section 3. A drift-flux model was implemented to predict interfacial drag, which is discussed in Section 4.

In addition to the findings related to void overprediction, CTF tends to greatly overpredict two-phase pressure drop when the Risø tests were added to the test matrix [44]. Lane [22] showed that the annular-mist flow regime interfacial drag model used in CTF significantly affects two-phase pressure-drop prediction. Lane [22] developed a model for annular-mist flow, which was previously implemented into CTF [33], that helped improve annular-mist pressure-drop prediction, but further analysis was performed in this work package. Improvements made for predicting two-phase pressure drop were improved in two ways: (1) the review and correction of the interfacial drag model in annular-mist flow, which is the primary flow regime encountered in the Risø tests, and (2) the inclusion of the two-phase multiplier model in the calibration activities. The first activity was a simple change and is briefly discussed in Section 5 .

The second, parallel approach to improving the CTF closure models involved calibrating the relevant 
closure models, which is consistent with the aforementioned models. The process that was used and the results are covered in Section 7.

In several sections, new models are assessed by comparing with experimental data. Frequently, the root-mean-square error (RMSE) is used for quantifying the scatter of CTF data to the experimental data. This is calculated as shown in Eq. (1), where $x$ is the value being compared between CTF and experiment (e.g., void fraction, wall temperature).

$$
R M S E=\sqrt{\sum\left(x_{C T F}-x_{e x p}\right)^{2} / N}
$$

Another statistic used for the assessment is the mean error, which is calculated as shown in Eq. (2).

$$
E_{\text {mean }}=\sum\left(x_{C T F}-x_{\text {exp }}\right) / N
$$




\section{VALIDATION MATRIX IMPROVEMENTS}

\subsection{Addition of New Tests}

To improve the two-phase closure models, as much experimental data as possible must be collected so that any model improvements can be thoroughly assessed. Furthermore, the data calibration efforts will require a wide range of data for performing the calibration. The existing CTF validation matrix included the following experimental datasets, which can be used for two-phase assessment.

- BWR Full Bundle Tests (BFBT) [23]: measurement of outlet void distribution and two-phase pressure drop in BWR bundle geometry.

- PWR Subchannel and Bundle Tests (PSBT) [30]: measurement of void in pressurized water reactor (PWR) single channels and PWR bundle geometry for high-void conditions.

- Risø [43]: measurement of two-phase pressure drop, film thickness, and film and droplet flow rate in tube and annular geometry.

- GE $3 \times 3$ [28]: measurement of outlet flow and quality distribution in a $3 \times 3$ bundle representative of BWR bundle geometry.

The full description of all tests and the baseline validation results are found in the CTF Validation and Verification Manual [35].

The validation matrix was expanded to include three new sets of tests as part of this work package, which can be used to validate the interfacial shear and surface heat transfer models that are being improved. These tests include the following:

- Bartolomei [14], [2]: measurement of void fraction at a range of operating conditions that covers BWR conditions. Adding these tests adds an additional set of void measurements to the validation matrix beyond the BFBT/PSBT tests that are currently added.

- New Experimental Studies of Thermal Hydraulics of Rod Bundles (NESTOR) [4]: measurement of wall temperature for single-phase and boiling heat transfer in PWR conditions. With plans to modify the boiling model to improve void prediction, rod surface temperature measurement data is necessary for ensuring that CTF temperature predictions are not simultaneously getting worse.

- Thom [38]: measurement of surface temperature in annular test geometry at PWR conditions. This dataset was used to develop the Thom boiling model, which is employed in CTF. It was added to ensure that there are no implementation errors with the Thom model and to act as another set of rod surface temperature measurement data.

The following sections summarize the Bartolomei and Thom tests that were added as part of this activity.

In addition to these three datasets mentioned, the following two-phase datasets were added as part of CASL-funded work [26] and are mentioned here for completeness:

- Westinghouse Advanced Loop Tester (WALT) [9]: measurement of surface temperature for single-phase and boiling flow at PWR conditions.

- Rohsenow tests [29]: measurement of surface temperature for two-phase conditions. 


\section{Table 1. Ranges of operating conditions for the Bartolomei tests}

\begin{tabular}{lrrr}
\hline Parameter & Minimum value & Maximum value & Unit \\
\hline Pressure & 15 & 148 & bar \\
Mass flux & 405 & 2,100 & $\mathrm{~kg} / \mathrm{m}^{2}-\mathrm{s}$ \\
Heat flux & 420 & 2,210 & $\mathrm{KW} / \mathrm{m}^{2}$ \\
Inlet subcooling & 11 & 140 & ${ }^{\circ} \mathrm{C}$ \\
\hline
\end{tabular}

\subsubsection{Bartolomei Tests}

2.1.1.1 Test description The Bartolomei experimental facility and tests are described in two articles by Bartolomei et al. [2] [14], which describe 38 tests that were modeled with two different base geometries: a $15.4 \mathrm{~mm}$ diameter vertical tube with a fixed length of $2,000 \mathrm{~mm}$ and a $12 \mathrm{~mm}$ vertical tube with varying lengths ranging from 800 to $1,500 \mathrm{~mm}$. The range of operating conditions is shown in Table 1 . These tests involve upward flow through a vertical cylindrical pipe with a constant applied heat flux over the entire pipe. Void fraction measurements are not available for 11 of the 38 cases, so only 27 cases are included. Of these cases, wall temperature and liquid temperature measurements are available for Case 1, in addition to cross-sectional void fraction measurements at different axial locations, which are provided for all 27 cases.

The system pressure and inlet liquid flow rate were measured by using a manometer and a differential manometer-flowmeter. Flow temperatures at the inlet and outlet of the experimental section were measured by using chromel-copel thermocouples. Flow temperature measurements are also available for Case 1 along the flow axis, as well as radial temperature profile measurements obtained by traversing the thermocouple at several points over the cross section of the flow. The temperature of the tube's outer wall was also measured by using the traversing thermocouples. The mean calometric temperature measurement is used for the Case 1 validation study.

Temperature measurements of the external surface of the heated wall tube are also available but were not used in the validation study. Heat flux is determined by measuring the electrical power supplied to the heaters. According to the study, for Cases 13-38, the maximum relative errors did not exceed 0.01 for pressure, 0.02 for mass velocity, and 0.03 for heat flux, and the maximum absolute error of temperature did not exceed $1 \mathrm{~K}$. The mean true vapor content (i.e., void fraction) was determined by penetrating gamma radiation from a ${ }^{170} \mathrm{Tm}$ source. Further details on the void fraction measurement, including the calibration process, are given in Bartolomei's articles [2] [14]. The maximum absolute errors in the void fraction measurements do not exceed 0.04 for Cases 13-38.

Experimental data used for this study include 27 conditions from the two tubular geometry test sections.

2.1.1.2 Validation with base CTF models The CTF models are created by using internal flow in a single channel with the inlet mass flux and inlet temperature used to set the inlet boundary conditions, and system pressure is used for the outlet boundary condition. Uniform heat flux is provided at the outer heater's wall surface, with the linear heat rate calculated to correspond to the experimental heat flux at the inner heater's surface outer internal tube surface. The 15.4 and $12 \mathrm{~mm}$ diameter test sections are divided into uniformly spaced axial nodes with a nodal size of $25 \mathrm{~mm}$. The Bartolomei data was presented in a way that did not associate measured void values with specific test sections or measurement locations. The actual 


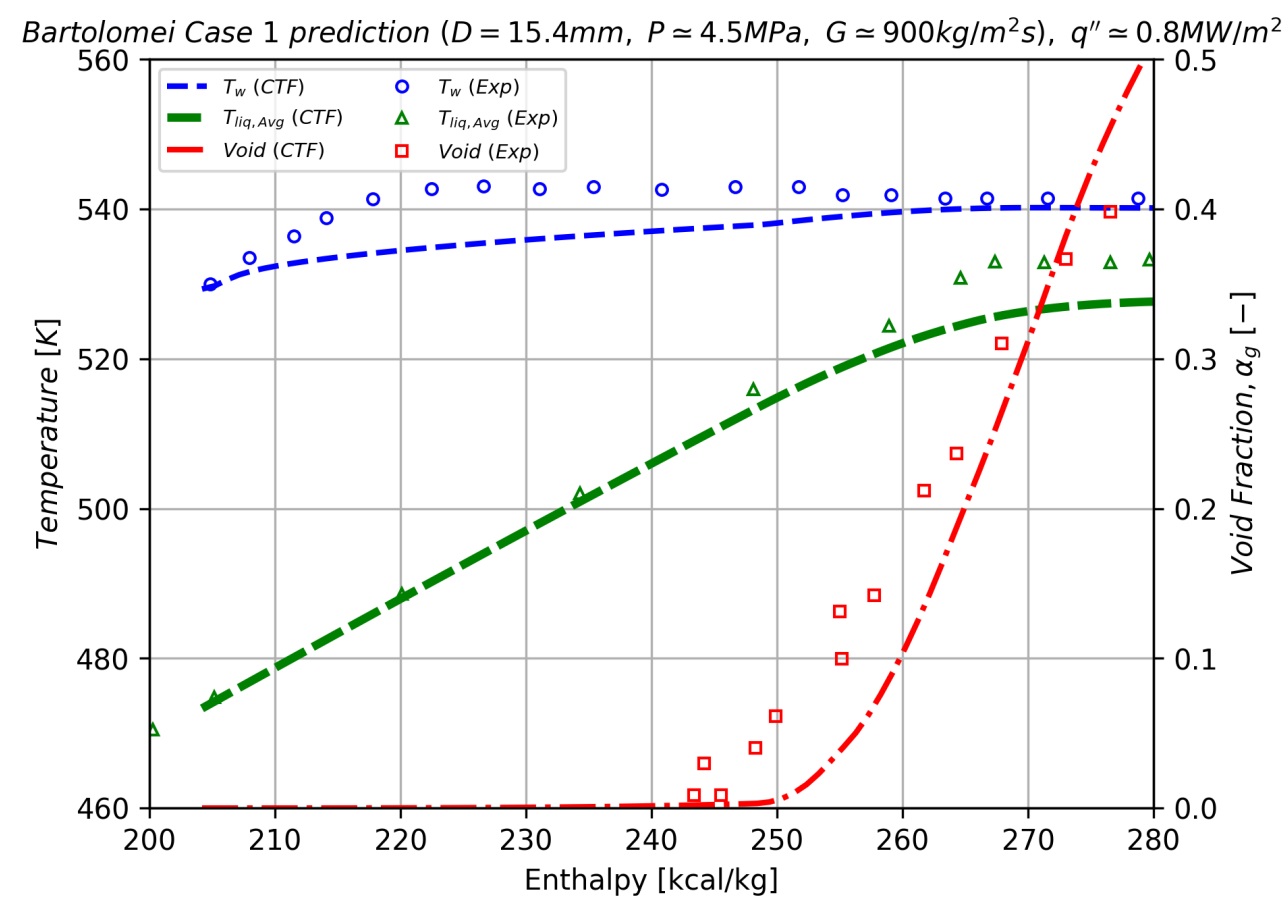

Figure 1. Comparison of CTF and experimental data of wall temperature, mean liquid temperature, and void fraction for Case 1.

measurement locations were unknown. However, the void measurement was given as a function of local quality, and the boundary conditions (i.e., inlet subcooling, inlet mass flux, heat flux, and outlet pressure) were known for the different test geometries. To model these in CTF, a fixed tube length of $1.5 \mathrm{~m}$ is used, which corresponds to a total of 60 nodes. Boundary conditions were applied as specified in the publication, and linear interpolation was performed on quality to match CTF void to experimental void. The current default subcooled boiling model, Thom with Hancox-Nicoll, is used for the initial modeling of these tests. The total number of nodes for Case 1 is 80 .

The results of Case 1 are shown in Figure 1. CTF captures the overall trend in the axial void development and liquid temperature profile reasonably well. However, CTF predicts the location of ONB at the very first node, with boiling occurring throughout the entire tube. Conversely, the experimental data indicates that ONB occurs further downstream. The experimental void fraction plateaus at the exit, whereas the CTF-predicted void fraction continues to rise as the liquid temperature plateaus, giving a constant temperature difference between the heater surface and the bulk liquid.

The overall void fraction comparison between the experimental data and CTF is shown in Figure 2. The model of Thom has considerable scatter on either side of the 1:1 line at lower void fractions with a consistent overprediction at higher void fractions similar to the other benchmarking studies presented in this section.

The results of the Bartolomei validation agree with existing CTF validation results when the mass flux is high. Void is overpredicted for all pressure levels in Figure 6, and Figure 2 indicates that more data points are overpredicted than underpredicted. However, the dataset reveals that at lower mass fluxes, void 


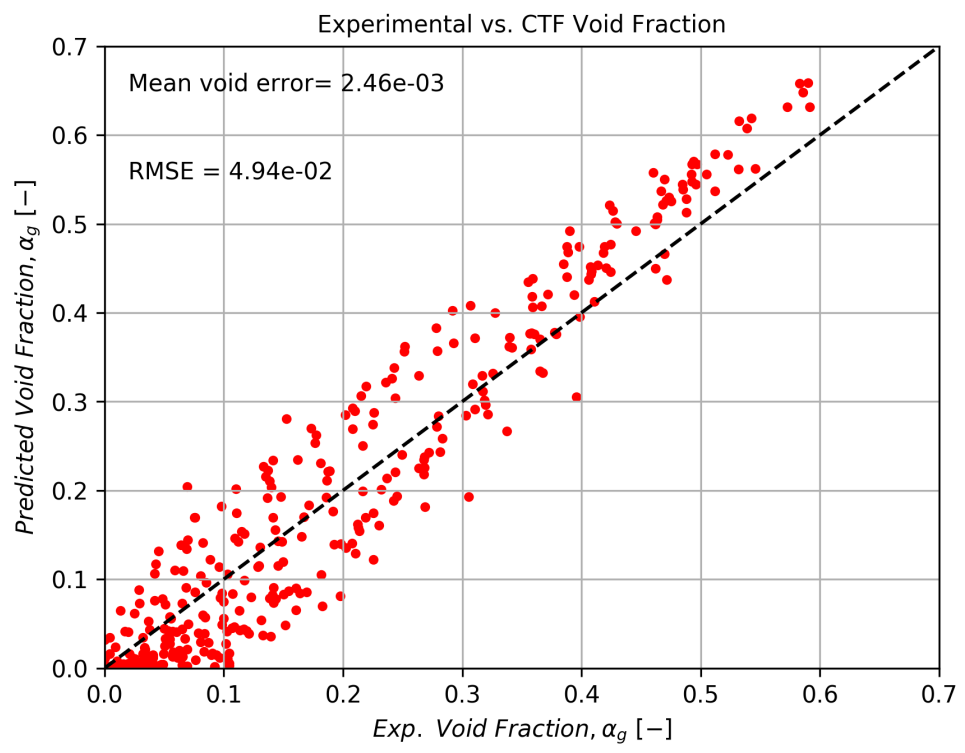

Figure 2. Overall comparison of CTF vs. experimental void fractions by using the subcooled boiling model of Thom.

becomes underpredicted. The BFBT void distribution cases were all run at a similar mass flux, so this trend does not appear in that dataset. A similar comparison should be made on the PSBT Series 5, 6, and 7 datasets to determine whether similar behavior exists. In PSBT Series 7, void predictions near the unheated rod were greatly overpredicted, which could be related.

Results of the parametric variation of key experimental system parameters from select cases are shown in the following four figures: mass flux variation in Figure 3, heat flux variation at 70 bar in Figure 4, and at 150 bar in Figure 5 and Figure 6. In the figures, the void fraction is plotted as a function of the thermodynamic equilibrium quality. Qualitatively, CTF void fraction predictions are better at higher mass fluxes and higher heat fluxes, and they are worse at lower mass fluxes. At both 70 bar and at 150 bar for varying heat fluxes and at moderate to high mass fluxes, the CTF predictions capture the void fraction development with underpredictions at low void fractions and overpredictions at high void fractions. The effect of pressure shown in Figure 6 is more pronounced in CTF than in the experimental data in terms of the ONB location and void fraction development. 


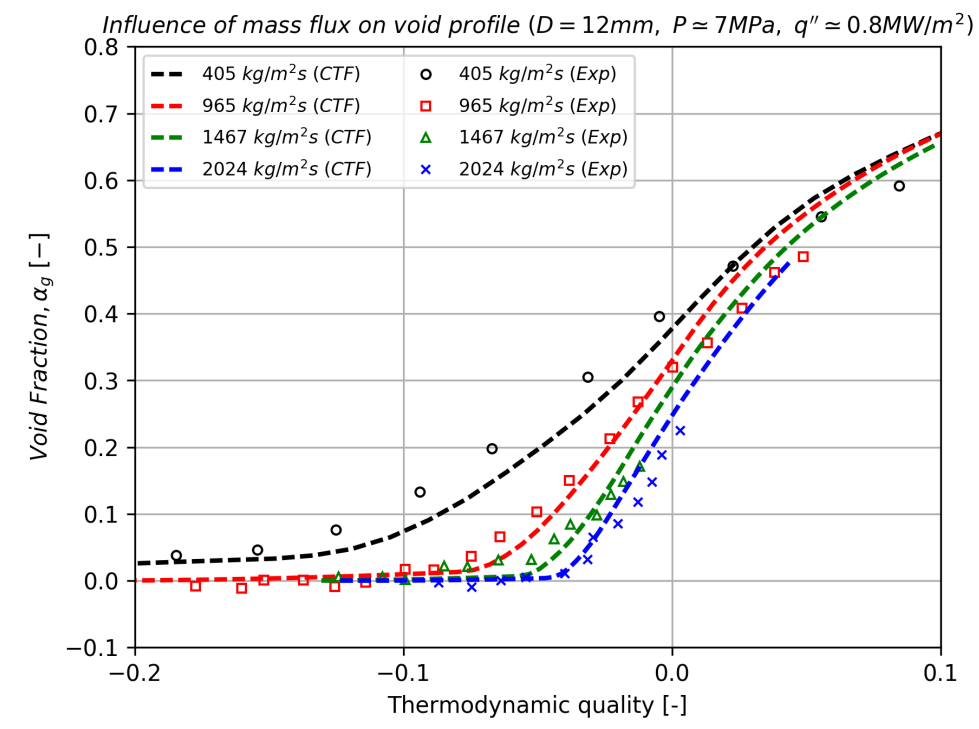

Figure 3. Comparison of CTF and experimental data for variation in mass flux at 70 bar and a heat flux of $0.8 \mathrm{MW} / \mathrm{m}^{2}$.

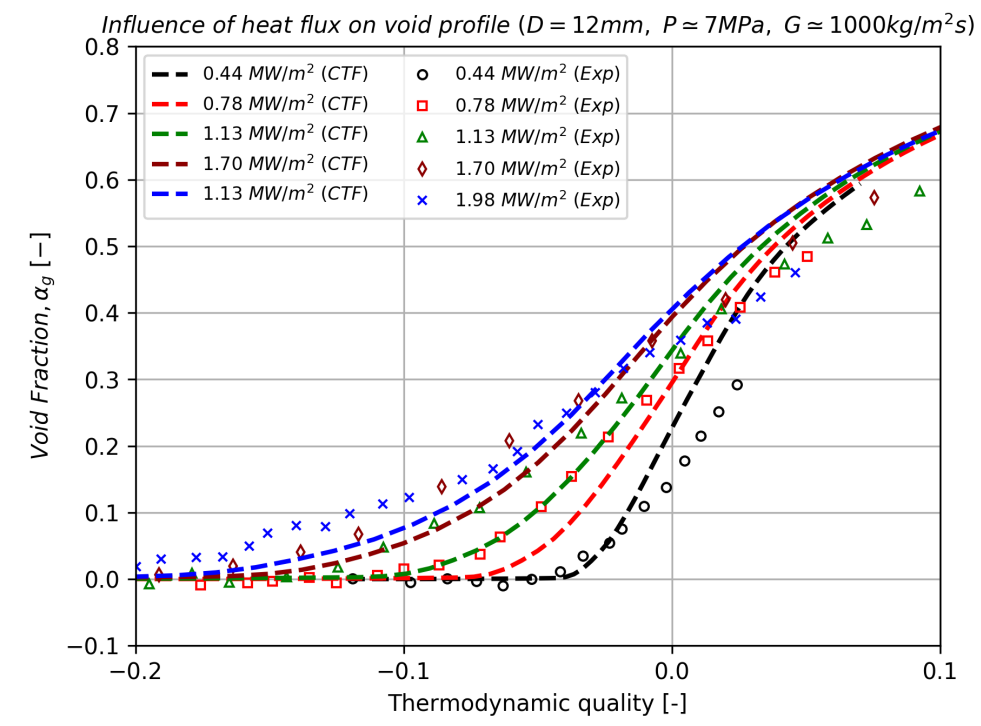

Figure 4. Comparison of CTF and experimental data for variation in mass flux at 70 bar and a mass flux of $1,000 \mathrm{~kg} / \mathrm{m}^{2}$-s. 


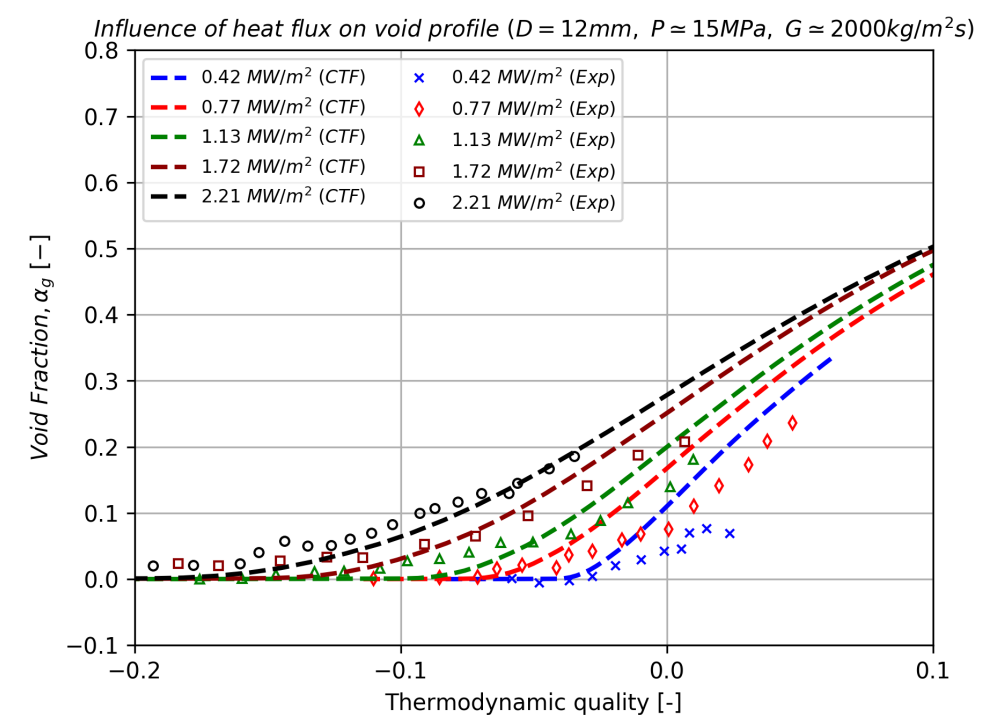

Figure 5. Comparison of CTF and experimental data for variation in mass flux at 150 bar and a mass flux of $2,000 \mathrm{~kg} / \mathrm{m}^{2}-\mathrm{s}$.

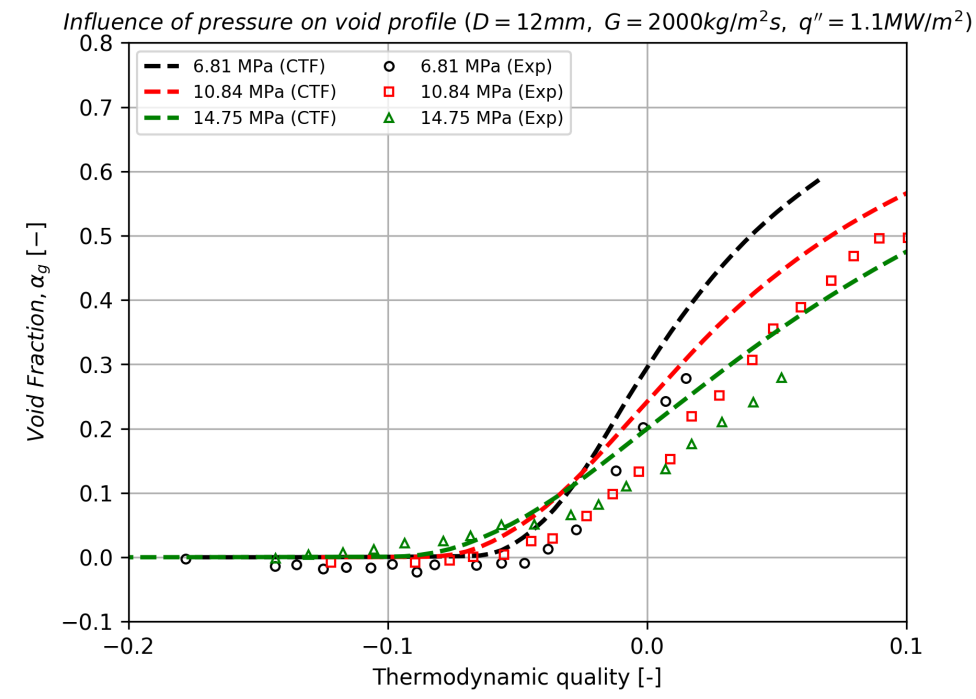

Figure 6. Comparison of CTF and experimental data for variation in system pressure at a mass flux of $2,000 \mathrm{~kg} / \mathrm{m}^{2}-\mathrm{s}$ and a heat flux of $1.1 \mathrm{MW} / \mathrm{m}^{2}$. 


\subsubsection{Thom}

2.1.2.1 Test description The Thom experimental facility and tests are described in Thom et al. [38]. Seven tests were modeled with the following annulus test section geometry: vertical tube with an inner diameter of $17.78 \mathrm{~mm}$, an outer diameter of $22.86 \mathrm{~mm}$, and a heated length of $3.66 \mathrm{~m}$. The purpose of this study is to test the implementation of the subcooled wall boiling model of Thom et al. [38]; therefore, the simulated tube length is $5 \mathrm{~mm}$ using one node. The inlet boundary conditions are set at the subcooled boiling regime, and the CTF wall temperature predictions are compared with data. The range of operating conditions are shown in Table 2. These tests involve upward flow with a uniform heat flux applied over the inner tube and outer tube. The metal thickness was arranged in the test section to obtain nearly equal heat flux on the inner and outer tubes. Heat flux was determined in the experiments by measuring the electrical power supplied to the heaters. Metal temperatures were measured by using thermocouples attached to the outer dry side of the tubes. The water-side metal temperatures were deduced by calculation. The bulk liquid temperatures were measured at the inlet and outlet of the test section by using thermocouples. Liquid temperatures along the test section were estimated by assuming a linearly proportional enthalpy gain. The system pressure and mass flux were held constant for all the conditions.

2.1.2.2 Validation with base CTF models Experimental data used for this study include seven conditions from an annular geometry test section. A single axial node is used in the simulation with a nodal size of $5 \mathrm{~mm}$, which is roughly equal to the hydraulic diameter. The CTF models are created by using internal flow in a single channel with the mass flux and liquid temperature set to match the experimental subcooling for all the data points that lie on or close to the subcooled boiling regime. The system pressure was used for the outlet boundary condition. Uniform heat flux is provided at the outer and inner heater wall surface with the linear heat rate calculated to correspond to the experimental heat flux at the respective surfaces, which are equal.

The results of the predicted and experimental delta wall temperatures are plotted in terms of liquid subcooling in Figure 7. Additionally, a comparison of predicted and experimental delta wall temperatures is shown in Figure 8, assuming an absolute experimental uncertainty of $5^{\circ} \mathrm{F}$ for all the experimental data points. The CTF results are generated by using the subcooled wall boiling model in Thom et al. [38]. Since the authors correlated their model to the experimental dataset, CTF predictions are expected to be within model uncertainty. The wall temperatures are overpredicted for several data points and underpredicted for one data point.The overprediction occurs near the transition point of the subcooled boiling regime, and a more accurate solution could be obtained by simulating the entire test section. Thus, it can be concluded that the wall boiling model from Thom et al. [38] is accurately implemented in CTF.

Table 2. Ranges of operating conditions for the Thom tests.

\begin{tabular}{lrrr}
\hline Parameter & Minimum value & Maximum value & Unit \\
\hline Operating pressure & 137.89 & 137.89 & $\mathrm{bar}$ \\
Mass flux & 971 & 1,025 & $\mathrm{~kg} / \mathrm{m}^{2}-\mathrm{s}$ \\
Heat Flux & 200 & 1,400 & $\mathrm{KW} / \mathrm{m}^{2}$ \\
\hline
\end{tabular}




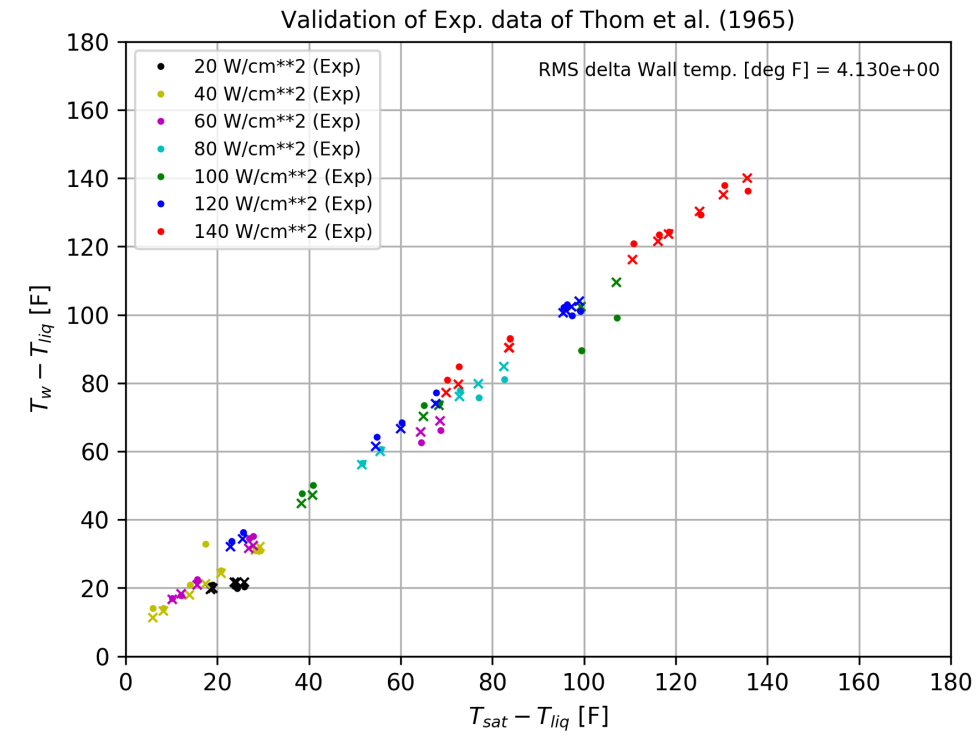

Figure 7. Comparison of predicted and experimental data from Thom for the temperature difference between the outer wall and the bulk liquid vs. liquid subcooling. The " $x$ " symbols are CTF predictions and the circles are experimental data.

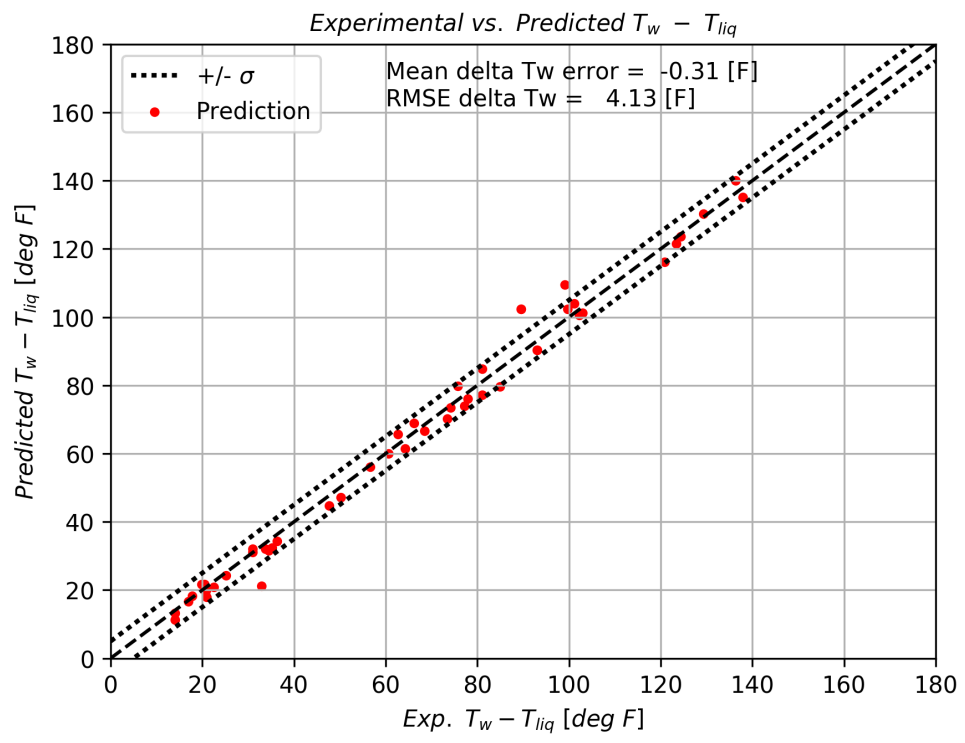

Figure 8. Comparison of predicted vs. experimental data from Thom for the temperature difference between the outer wall and the bulk liquid. 


\subsubsection{NESTOR Tests}

The NESTOR tests were performed in a $5 \times 5$ electrically heated rod bundle operated at prototypical PWR operating conditions. The geometry and operating conditions are described in Wells, Peturaud, and Yagnik [41]. The experiments were divided into two classes: unheated and heated. Both classes used a similar geometry that used mixing vane grids for the spacer grids and placed simple support grids midway in the grid spans to maintain geometric integrity. For this work, the heated tests were of interest for validating the CTF heat transfer model. The geometry of the heated tests is shown in Figure 9. The heater rods in the facility were tube geometry, and the inner nine rods were instrumented with one sliding thermocouple per tube. This allowed temperature measurements to be made on the inside surface of the tube during the experiment. Because the thermocouples were mobile, a high-fidelity rod surface temperature map was obtained for each experiment. The heated tests were operated at both single-phase and boiling conditions, which allows single-phase and subcooled boiling heat transfer models to be assessed in CTF and also grid enhancement models.

When the axial mesh was designed for use in CTF, a mesh bound was placed at the bottom and top of each grid, so the spacer grid regions were not subdivided in the mesh. The grid level is not split, so there is one mesh level per grid. This is the typical way for generating CTF meshes for use in VERA simulations. A mesh cell height target of $2.5 \mathrm{~cm}$ was used to construct the axial mesh, and local mesh cell heights were adjusted to ensure that grids were placed in their correct axial locations. This resulted in 151 axial mesh cell levels being used in the model. This is considered a fine mesh for CTF. A typical mesh size for a full-length core in VERA includes about 50 levels. Because the NESTOR experimental results are not public, the analysis is not shown here. However, a comparison of validation error will be presented in response to model improvements later in Section 6.6.

\subsection{Validation Matrix Driver}

The CTF validation matrix has been developed with a strong emphasis on the automation of test modeling and analysis. For each test that was added to the test matrix, a set of Bash and/or Python scripts were included to generate CTF inputs, run the tests, and generate plots and statistics to summarize the comparison between CTF and experimental data. Although each category of validation tests includes a set of such scripts to help model building and/or analyze the test results and comparing them with experimental data, the automation for each test set was typically performed in a custom way. This proved to be problematic since the test matrix continued to grow over time because each test set requires a slightly different set of steps to reperform the analysis. Because the authors intended to make rapid changes to CTF closure models, it was necessary to develop an approach that would allow the test results to be rapidly executed and analyzed after changing the code.

To address this issue, a validation matrix driver utility was developed. This Python utility includes two core components:

1. ValidationTest: a base class that defines a set of tests. It is intended that the developer (i.e., test implementer) will extend this class for a test set that is added to the test matrix. This class includes methods that can be called to find tests in the test set, process test results, extract summary statistics, and rebaseline gold results files for regression tests in the set. 

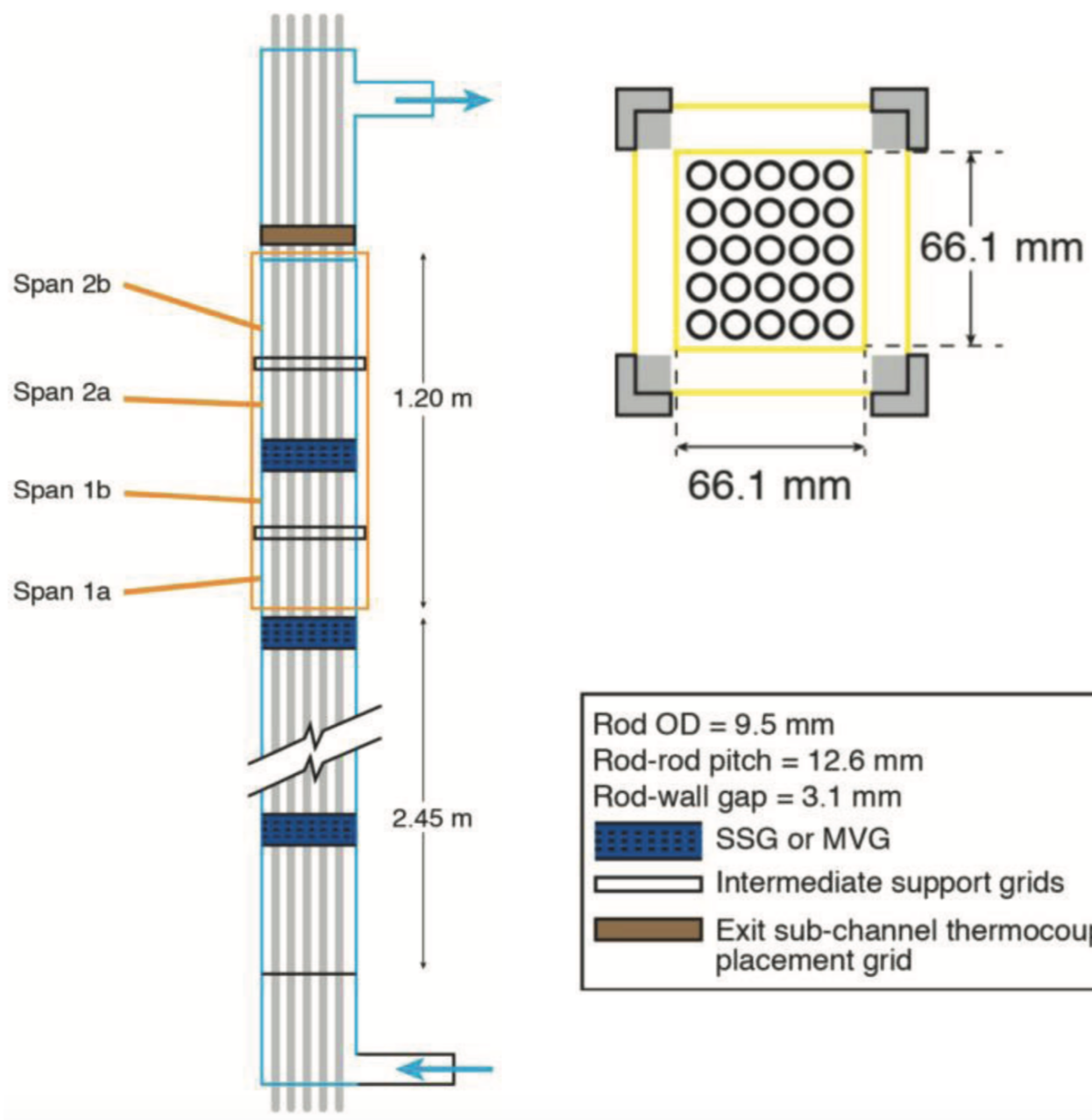

Rod OD $=9.5 \mathrm{~mm}$

Rod-rod pitch $=12.6 \mathrm{~mm}$

Rod-wall gap $=3.1 \mathrm{~mm}$

DSG or MVG

Intermediate support grids

$\square$ Exit sub-channel thermocouple placement grid

Figure 9. Geometry of the heated NESTOR tests. 
2. runMatrix: the driver script that runs all tests in the matrix. The utility will walk the test matrix directory structure in the CTF repository and will find all tests registered by the developer (class extensions of the ValidationTest abstract class). The driver will run tests in parallel on a personal computer or a high-performance computing (HPC) system via a job queuing system. The driver script is set up to use multiple nodes on an HPC system, which allows the entire CTF validation matrix to be run in a short amount of time. The driver will finally generate summary statistics that give a high-level overview of how test results have changed from baseline results, generate new figures for the CTF Validation Manual, and rebaseline results of regression tests while detailing the changes. This driver will also detect any cases that do not converge and will summarize any encountered errors during the process of running and analyzing tests.

So far, all major two-phase validation tests have been added to the testing matrix driver by using this approach. All the new datasets added as part of this work package have also been added to the test matrix by using this utility. The usage instructions from the driver utility summarize the options available to the script user.

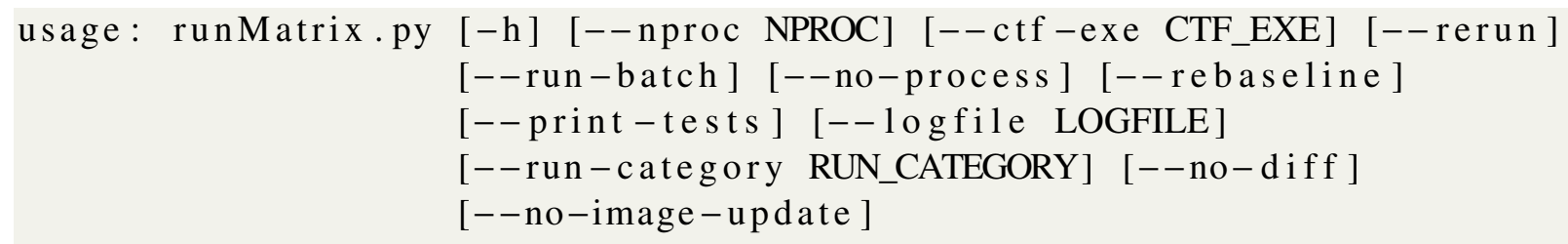

Runs the CTF validation matrix. It is assumed this script is run in the source directory. By default, this will run every validation test, perform all data processing steps that compare experimental and calculated results including generation of plots, diff all tests to gold files if that test is included as a regression test in the CTF CI, and make a printout summarizing which tests did not converge (if any) and what the differences were from gold files. All tests will be run in parallel according to user provided number of processors. Note that this driver script depends on other driver scripts defined by the developer for each test category. Adding new tests requires the developer to add new driver scripts that define the steps of running the tests, processing results, etc. The ValidationTest base class should be used to define the process of running a set of tests - See ValidationTest.py for more details.

optional arguments:
$-\mathrm{h},--\mathrm{help}$
show this help message and exit
-- nproc NPROC
Number of processors to use for the job.
$--\mathrm{ctf}-\mathrm{exe}$ CTF_EXE
The path to the CTF exe that will be used to
run the tests. If not specified, the tests will not be run.
-- rerun
By default, the driver will skip tests that
have a '.success' file which indicates the test was previously run successfully. Specify this flag to rerun all tests; even ones that were previously run successfully.
--run-batch Run all jobs using the PBS job scheduler (ross 


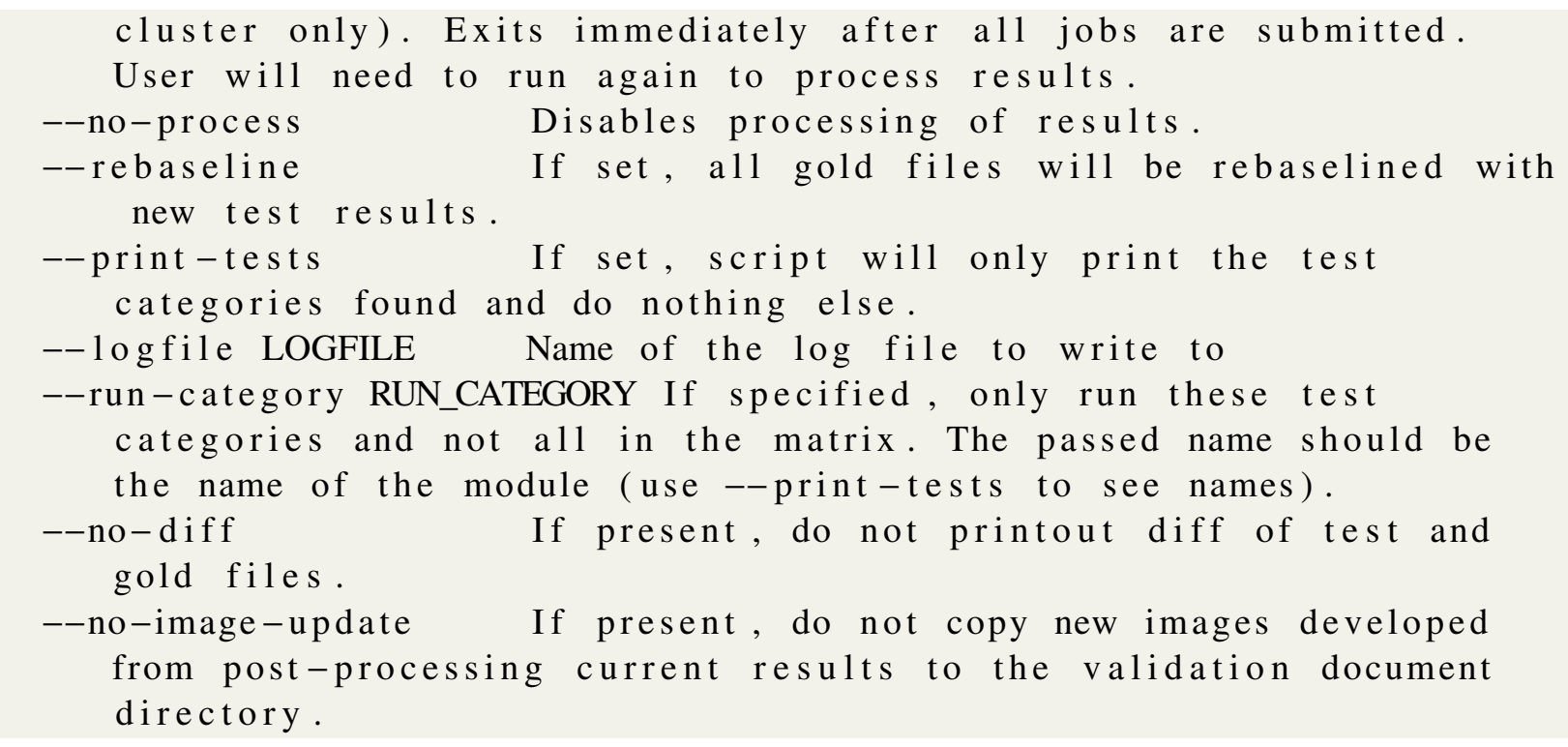




\section{SUBCOOLED BOILING MODEL IMPROVEMENTS}

CTF includes models for both pre- and post-critical heat flux (CHF) heat transfer mechanisms. The heat transfer regime is determined for every fluid cell in the model that connects to a solid surface. In the original CTF model, if the solid surface temperature is greater than the local saturation temperature and less than the local CHF temperature, then a boiling heat transfer model will be used. If the local enthalpy exceeds the local liquid saturation enthalpy, then a saturated boiling heat transfer model will be used; if not, then a subcooled boiling model will be used. In practice, the same boiling model is used for both subcooled and saturated boiling conditions, with the only difference being in how vapor generation is determined. During subcooled boiling, a certain amount of void generated at the solid surface will immediately collapse back to liquid, depending on local thermal hydraulic conditions. The amount of void generated in subcooled boiling is determined by using a near-wall condensation model. The boiling models originally available in CTF were Thom and Chen, with the Hancox-Nicoll model used for near-wall condensation. These models, along with the full details of the heat transfer regime selection logic, are described in the CTF Theory Manual [34].

The approach for determining vapor generation heat flux during subcooled boiling involves calculating the total heat flux due to boiling by using either Thom or Chen and then subtracting the heat flux that is delivered to the liquid in the form of latent heat (when bubbles condense back to liquid) calculated by using the Hancox-Nicoll near-wall condensation model. By dividing this heat flux by the latent heat of vaporization, the total steaming rate on the solid surface can be determined. Past reviews of this model have questioned its validity, and it was shown in Zhao et al. [45] that it significantly impacts void prediction in PSBT tests.

A common alternative approach for modeling boiling heat transfer and vapor generation involves using an ONB model to determine when boiling begins in conjunction with a critical enthalpy model to determine when vapor begins to detach from the wall. This approach is used in both TRACE [1] and THERMIT-2 [19], two other thermal-hydraulic fluid codes developed for nuclear system analysis, which were reviewed as part of this work package.

For this work, the approach used by TRACE was implemented into CTF and tested for the CTF validation matrix. This involved three primary changes in CTF. First, the Basu ONB criteria [3] are used to determine which surfaces are experiencing boiling heat transfer. Second, the Gorenflo heat transfer model [15] is used to determine the heat flux in subcooled and saturated boiling conditions. Third, the Saha-Zuber model [31] is used to determine the enthalpy at which bubbles begin to detach and contribute to void production. The model form and results that use this new model described as follows were also submitted for publication to the American Nuclear Society (ANS) Winter Meeting conference [20].

\subsection{Basu ONB criteria}

The ONB criteria are used to determine when boiling occurs. Rather than starting the boiling heat transfer immediately when the wall temperature rises over local saturation temperature, it will be delayed until the wall temperature rises over the ONB superheat-some temperature that is higher than the local saturation temperature. The Basu model considers that boiling will start in cavities in the surface material of the wall, and it will depend on surface wettability and cavity size. The available cavity diameter, $D_{\mathrm{c}}$, is proportional to a cavity diameter, $D_{\mathrm{c}}^{\mathrm{o}}$ that corresponds to the tangency condition and a wettability parameter, $F(\phi)$. The wall superheat at ONB can be expressed as follows: 


$$
\Delta T_{\mathrm{onb}}=\frac{\sqrt{2}}{F(\phi)} \sqrt{\frac{\sigma T_{\mathrm{sat}} q_{\mathrm{w}}^{\prime \prime}}{\rho_{g} h_{\mathrm{fg}} k_{\mathrm{f}}}},
$$

where $q_{\mathrm{w}}$ is the wall heat flux. The empirically obtained wettability parameter is a function of the fluid-surface contact angle, $\phi$, which for the combination of water and stainless-steel is 38 degrees [3]. In the current implementation of the model, this value was hard-coded into the model. In the future, it would be interesting to expose this as a user-defined parameter and test the sensitivity of the results to this value. Eq. (3) is an implicit equation as the wall superheat, $\left(\Delta T_{o n b}=T_{o n b}-T_{\text {sat }}\right)$, the wall heat flux. An explicit relationship can be obtained by first defining the wall heat flux as follows:

$$
q_{\mathrm{w}}^{\prime \prime}=h_{\mathrm{fc}}\left(T_{\mathrm{onb}}-T_{1}\right)
$$

By substituting Eq. (4) in Eq. (3), a quadratic equation is obtained. Solving the quadratic equation and ignoring the negative root, the minimum wall temperature for boiling incipience can be obtained as follows:

$$
T_{\text {onb }}=T_{\text {sat }}+0.5 \Delta T_{\text {sat,onb }}+0.5 \sqrt{\Delta T_{\text {sat,onb }}^{2}+4 \Delta T_{\text {sat,onb }} \Delta T_{\text {sub }}}
$$

where $\Delta T_{\text {sub }}$ is the local liquid subcooling. Finally, $\Delta T_{\text {sat }}$, is defined as follows:

$$
\Delta T_{\text {sat,onb }}=\frac{2 h_{\mathrm{fc}} \sigma T_{\mathrm{sat}}}{F_{(\phi)}^{2} \rho_{g} h_{\mathrm{fg}} k_{\mathrm{f}}} .
$$

\subsection{Gorenflo Heat Transfer Model}

The Gorenflo heat transfer model is used for determining the heat transfer in subcooled and saturated boiling heat transfer regimes. It is used when CTF determines that a surface is in a pre-CHF boiling heat transfer regime. In this way, the model is similar to the Chen and Thom models that were already implemented into CTF.

The Gorenflo model is based on a reduced heat transfer coefficient, which is proportional to a reference state heat transfer coefficient and factors representing the influence of the wall heat flux, reduced pressure (ratio of system pressure to critical pressure), and wall properties. The Gorenflo pool boiling heat transfer coefficient is given as follows:

$$
h_{\mathrm{pb}}=h_{o} F\left(p^{*}\right) F_{w}\left(\frac{q^{\prime \prime}}{q_{o}^{\prime \prime}}\right)^{n},
$$

where $h_{\mathrm{o}}$ is the reference heat transfer coefficient, $F\left(\mathrm{p}^{*}\right)$ is the influence of the reduced pressure where $p^{*}$ is the reduced pressure, $F_{\mathrm{w}}$ is the influence of wall properties, and the wall heat flux factor is defined in terms of the reference heat flux, $q_{\mathrm{o}}^{\prime \prime}$, where $n$ is a function of the reduced pressure. The expressions for $h_{\mathrm{o}}, n$ and $F\left(\mathrm{p}^{*}\right)$ are chosen for water from Gorenflo [15] and described in Salko et al. [34]. The surface roughness factor, $F_{\mathrm{w}}$, is taken as unity in this analysis for simplification. The superposition of the forced convective component and the pool boiling heat flux to obtain the flow boiling heat flux is calculated based on the 
power-law or flow additive approach. In CTF, an exponent of three is used (similar to the US Nuclear Regulatory Commission technical report [8]), as proposed by Steiner and Taborek [36], wherein, the flow boiling heat flux, $q_{\mathrm{nb}}^{\prime \prime}$, is the cubic root of the superposition of the single-phase forced convective heat flux, $q_{\mathrm{fc}}^{\prime \prime}$ (cubed) and the pool boiling heat flux, $q_{\mathrm{pb}}^{\prime \prime}$ (cubed). This approach is as follows:

$$
q_{\mathrm{nb}}^{\prime \prime}=\left(q_{\mathrm{fc}}^{\prime \prime 3}+q_{\mathrm{pb}}^{\prime \prime 3}\right)^{(1 / 3)}
$$

This approach is modified to the superposition of heat fluxes since it is applied to subcooled and saturated boiling heat transfer regimes. Additionally, there is a sharp rise in the nucleate boiling heat flux when it is suddenly triggered at the onset of nucleate boiling. To smooth the transition, the approach of Bjorge, Hall, and Rohsenow [5] is used. Based on this approach, the pool boiling heat flux using $T_{\mathrm{ONB}}$ is subtracted from the pool boiling heat flux at the local surface temperature, $q_{\mathrm{pb}}^{\prime \prime}$. Therefore, the total nucleate boiling heat flux, $q_{\mathrm{nb}, \mathrm{t}}^{\prime \prime}$, is given as follows:

$$
q_{\mathrm{nb}, \mathrm{t}}^{\prime \prime}=\left(q_{\mathrm{fc}}^{\prime \prime 3}+\left(q_{\mathrm{pb}}^{\prime \prime}-q_{\mathrm{bi}}^{\prime \prime}\right)^{3}\right)^{(1 / 3)}
$$

where $q_{\mathrm{bi}}$ is the pool boiling heat flux at the point of boiling initiation, and $q_{\mathrm{fc}}$ is the forced convective heat flux. $q_{\mathrm{bi}}$, which is calculated by using the wall temperature at the onset of nucleate boiling. To calculate the net heat flux available for vapor generation, the contribution of the forced convective heat transfer must be subtracted. Because it is difficult to isolate the contribution of the forced convective heat flux from Eq. (8), a simple approximation is used. Finally, the nucleate boiling heat transfer coefficient can be obtained by dividing Eq. (9) by $T_{\text {wall }}-T_{\text {liq }}$.

$$
q_{\mathrm{nb}}^{\prime \prime} \simeq q_{\mathrm{nb}, \mathrm{t}}^{\prime \prime}-q_{\mathrm{fc}}^{\prime \prime}
$$

\subsection{Bubble Detachment Criteria}

In subcooled boiling, only a fraction of the nucleate boiling heat flux is available for vapor generation since near-wall condensation results in a fraction of the generated vapor condensing back into liquid. Near-wall condensation is accounted through a bubble detachment enthalpy, $h_{c r}$, which is based on the model of Saha-Zuber [31]. According to the Saha-Zuber model, the balance between evaporation at the wall and near-wall condensation is estimated by using a similarity parameter, which is a function of the mass flux. By using the bubble detachment enthalpy, the fraction of vapor generation in subcooled boiling is determined via the mechanistic model of Lahey [21]. The vapor generation fraction is calculated by estimating the excess liquid enthalpy, $h_{l}$, over the critical liquid enthalpy as a proportion of excess saturated liquid enthalpy, $h_{f}$, to the critical enthalpy, $h_{\mathrm{cr}}$, given as follows:

$$
F_{\text {gam }}^{*}=\frac{\left(h_{1}-h_{\mathrm{cr}}\right)}{\left(h_{\mathrm{f}}-h_{\mathrm{cr}}\right)}
$$

In subcooled boiling, the vapor that leaves the superheated layer is replaced by subcooled liquid from the bulk that must be heated. This pumping effect for subcooled boiling is obtained by a model proposed by 
Rouhani, which is similar to the default CTF model. The effective vapor generation fraction, $F_{\mathrm{gam}}^{*}$, is obtained by multiplying Eq. (10) with Eq. (11).

$$
\epsilon_{\Gamma}=\frac{h_{\mathrm{fg}}}{h_{\mathrm{fg}}+\left(h_{f}-h_{l}\right) \frac{\rho_{f}}{\rho_{g}}} .
$$




\section{BUBBLY FLOW INTERFACIAL DRAG}

\subsection{Background}

The two options for performing a general two-phase flow problem solution include using a two-fluid model or a drift-flux model, depending on the desired degree of the dynamic coupling between the phases. The drift-flux is an approximate formulation in comparison with the more rigorous two-fluid formulation, which allows for more independent motion of the phases (e.g., counter-current flow) and couples the phases through interfacial shear and heat transfer closure relationships. In view of the practical importance and simplicity of the model for two-phase flow analysis, it has been studied extensively. In the state of the art, the constitutive equations for the drift-flux model have been well-developed for vertical upward dispersed two-phase flow and proven to be very accurate in predicting void fraction. These kinematic constitutive relations for the drift-flux velocity obtained for this model have been often used in two-fluids computational T/H codes — such as TRACG [10], TRACE [1], and THERMIT-2 [19]— to describe the interfacial drag in vertical dispersed upward flow, which in turn will define the relative phasic velocity and void fraction prediction. To date, recently performed CTF validation data have shown that the code tends to overpredict the void fraction for these conditions. This suggests that the interfacial was overpredicted for these conditions. To improve the code predictive capability, an option was added that reformulates the interfacial drag based on the drift-flux approach. The derivation of the model as implemented in CTF is shown in the remainder of this section. An assessment of the model is performed in Section 6.

\subsection{Drift Flux Model}

In subchannel analysis, various thermal hydraulic quantities will be expressed in term of the cross-sectional average value. Some nomenclature to assist with this is first defined. Following the same notation as in Ishii [17], a simple area average for a general variable $F$ is defined by:

$$
\langle F\rangle=\frac{1}{A} \int_{A} F d A
$$

The cross-sectional area is given as $A$. The void fraction-weighted mean value is given by:

$$
\left\langle\left\langle\alpha_{k} F\right\rangle\right\rangle=\frac{\left\langle\alpha_{k} F\right\rangle}{\left\langle\alpha_{k}\right\rangle} .
$$

The term $k$ represents the phase and can be either $l$ or $g$. Thus, the void-weighted mean vapor and liquid velocity are defined by:

$$
\begin{aligned}
\left\langle\left\langle\alpha_{g} v_{g}\right\rangle\right\rangle & =\frac{\left\langle\alpha_{g} v_{g}\right\rangle}{\left\langle\alpha_{v}\right\rangle} \\
\left\langle\left\langle\left(1-\alpha_{g}\right) v_{l}\right\rangle\right\rangle & =\frac{\left\langle\left(1-\alpha_{g}\right) v_{g}\right\rangle}{\left\langle 1-\alpha_{g}\right\rangle} .
\end{aligned}
$$


The $v_{l}$ and $v_{g}$ terms represent the liquid and vapor phase velocity, respectively. By using the Zuber definition [46], the phase volumetric fluxes for liquid $\left(j_{l}\right)$ and vapor $\left(j_{g}\right)$ are defined as:

$$
\begin{aligned}
& j_{l}=\left(1-\alpha_{g}\right) v_{l} \\
& j_{g}=\alpha_{g} v_{g} .
\end{aligned}
$$

The $j_{l}$ and $j_{g}$ terms are also known as the superficial velocities for liquid and vapor. The $v_{l}$ and $v_{g}$ terms are defined as the volumetric flux of the phase divided by the cross-sectional area the phase occupies $\left(A * \alpha_{k}\right)$. The total volumetric flux of the mixture is then defined as the sum of the individual phase superficial velocities.

$$
j=j_{l}+j_{g}=\alpha_{g} v_{g}+\left(1-\alpha_{g}\right) v_{l}
$$

The local vapor drift velocity is defined as:

$$
\begin{aligned}
v_{g j}=v_{g}-j & =v_{g}-\alpha_{g} v_{g}-\left(1-\alpha_{g}\right) v_{l} \\
& =\left(1-\alpha_{g}\right)\left(v_{g}-v_{l}\right)=\left(1-\alpha_{g}\right) v_{r} .
\end{aligned}
$$

The relative velocity can then be defined as:

$$
V_{r}=\frac{v_{g j}}{1-\alpha_{g}}
$$

The weighted relative velocity is given by:

$$
\bar{V}_{r}=\frac{\left\langle v_{g j}\right\rangle}{\left\langle 1-\alpha_{g}\right\rangle} .
$$

By using the above definitions, Zuber proposed the following form for representing weighted mean velocity as a function of volumetric flux and vapor drift velocity:

$$
\left\langle\left\langle v_{g}\right\rangle\right\rangle=C_{0}\langle\langle j\rangle\rangle+\left\langle\left\langle v_{g j}\right\rangle\right\rangle .
$$

The term $C_{0}$ is known as the void distribution parameter and was defined as:

$$
C_{0}=\frac{\left\langle\alpha_{g} j\right\rangle}{\left\langle\alpha_{g}\right\rangle} .
$$

By combining Eq. (19) and Eq. (20), one can get the final form of the relative velocity:

$$
\bar{V}_{r}=\frac{1-C_{0}\left\langle\alpha_{g}\right\rangle}{\left(1-\left\langle\alpha_{g}\right\rangle\right)}\left\langle\left\langle v_{g}\right\rangle\right\rangle-c_{0}\left\langle\left\langle v_{l}\right\rangle\right\rangle
$$


The relative velocity defined in Eq. (22) establishes the first link between the drift-flux approach and the two-fluid model through the distribution parameter $C_{0}$. This relative velocity and the CTF velocity predicted by using the legacy interfacial drag models might differ greatly. Generally, the CTF models predict a higher interfacial drag, which reduces phase slip and in turn increases predicted void. The model shown by Eq. (22) is describing the relative phase velocity predicted by the drift-flux model, which cannot be directly used by the two-fluid model in CTF to set phase velocity distribution. Rather, this model must be used to define an interfacial drag force that CTF will use in the solution of the vapor and liquid momentum equations. This will drive the velocity distribution to match the one predicted by the drift-flux model. The following section covers the calculation of this interfacial drag force from the drift-flux velocity.

\subsection{Interfacial Drag Model Form}

In the drift-velocity approach, the drag coefficient is formulated from a local steady-state force balance in the flow direction between buoyancy and interfacial drag forces for the gas and liquid phase. The liquid and gas pressure are assumed to be equal. The interfacial drag per unit volume is then equal to the buoyancy force.

$$
\alpha_{g}\left(1-\alpha_{g}\right) g \Delta \rho=\tau_{i, l g}^{\prime \prime \prime},
$$

where parameters $\Delta \rho$ and $g$ are the density difference between the phases and gravitational acceleration.

In bubbly flow, the standard drag force per unit volume that acts on multiple particles under steady-state conditions can be given in terms of the drag coefficients $C_{d}$ and interfacial area concentration $A_{i}^{\prime \prime \prime}$ and is based on the relative velocity as:

$$
\tau_{i, l g}^{\prime \prime \prime}=\frac{1}{8} C_{d} A_{i}^{\prime \prime \prime} \rho_{l}\left|\bar{V}_{r}\right| \bar{V}_{r}
$$

The interfacial area of a single spherical bubble will be the bubble surface area divided by the bubble volume. This is then multiplied by the volume fraction to account for the total volume occupied by the bubbles. When multiplied by volume of the control volume, it will return ineterfacial area concentration in the volume. In equation form, this translate to:

$$
A_{i}^{\prime \prime \prime}=\frac{\alpha_{g} 4 \pi r^{2}}{4 / 3 \pi r_{b}^{3}}=\frac{3 \alpha_{g}}{r_{b}} .
$$

Substituting this into Eq. (24) yields the following:

$$
\tau_{i, l g}^{\prime \prime \prime}=\frac{3}{8} \frac{C_{d} \alpha_{g} \rho_{l}\left|\bar{V}_{r}\right| \bar{V}_{r}}{r_{b}} .
$$

This can be substituted back into Eq. (23) to obtain an expression for the bubble drag coefficient with respect to the relative velocity: 


$$
C_{d}=\frac{8}{3} \frac{\left(1-\alpha_{g}\right) \Delta \rho g r_{b}}{\rho_{l} \bar{V}_{r}^{2}} .
$$

The coefficient of drag on the bubbles is now a function of the relative velocity, which will be defined by the kinematic constitutive relation of the drift flux model. Eq. (19) can be substituted into Eq. (27) to obtain $C_{d}$ as a function of the drift velocity:

$$
C_{d}=\frac{8}{3} \frac{\left(1-<\alpha_{g}>\right)^{3} \Delta \rho g r_{b}}{\rho_{l}<v_{g j}^{2}>} .
$$

The next steps are to define the bubble radius and drift velocity, $v_{g j}$. The bubble radius is defined as a function of the critical Webber number, $W e_{c r i t}$, similar to what is already done in CTF:

$$
r_{b}=\frac{\sigma W e_{c r i t}}{2 \rho_{l} \bar{V}_{r}^{2}} .
$$

The surface tension is $\sigma$. The drift velocity is often given as a function of a modeling coefficient, $k$, as shown:

$$
v_{g j}=k\left(\frac{\sigma \Delta \rho g}{\rho_{l}^{2}}\right)^{\frac{1}{4}}
$$

Substituting Eq. (29) into Eq. (26) leads to:

$$
\tau_{i, l v}^{\prime \prime \prime}=\frac{3}{4} \frac{C_{d}}{W e_{c r i t}} \frac{<\alpha_{g}>\rho_{l}^{2}\left|\bar{V}_{r}^{3}\right|}{\sigma} \bar{V}_{r}
$$

The ratio $\frac{C_{d}}{W e_{c r i t}}$ is obtained by substituting equations Eq. (29) and Eq. (30) into Eq. (28):

$$
\frac{C_{d}}{W e_{\text {crit }}}=\frac{4}{3} \frac{\left(1-<\alpha_{g}>\right)^{5}}{k^{4}} .
$$

Substituting Eq. (32) into Eq. (31) gives the final form of the interfacial shear:

$$
\tau_{i, l v}^{\prime \prime \prime}=\frac{1}{k^{4}} \frac{<\alpha_{g}>\left(1-<\alpha_{g}>\right)^{5} \rho_{l}^{2}\left|\bar{V}_{r}^{3}\right|}{\sigma} \bar{V}_{r}
$$

The last step is to define the $k$ coefficient, which is going to be flow regime-dependent. In the small bubble regime, the leading constant $k$ of the drift velocity given by Eq. (30) is constant, ranging from $\sqrt{2}$ to 1.57 . Ishii [17] recommended using $\sqrt{2}$ for the dispersed bubble flow regime and 1.57 for the dispersed droplet flow regime. A value of $\sqrt{2}$ is initially selected for CTF. 
As the void fraction increases, agglomeration becomes progressively more important and the bubbles grow to form either Taylor cap or slug bubbles. For this regime, Kataoka and Ishii [18] developed one correlation for the drift velocity with two parts to handle both cases of cap bubble and slug flow. The leading constant $k$ is given by:

$$
k_{l b}= \begin{cases}0.0019\left(D_{H}^{\star}\right)^{0.809}\left(\frac{\rho_{g}}{\rho_{l}}\right)^{-0.157}\left(N_{\mu_{l}}\right)^{-0.562} & \text { if } D_{H}^{\star} \leq 30 \\ 0.03\left(\frac{\rho_{g}}{\rho_{l}}\right)^{-0.157}\left(N_{\mu_{l}}\right)^{-0.562} & \text { if } D_{H}^{\star}>30,\end{cases}
$$

where the nondimensional hydraulic diameter $D_{H}^{\star}$ is defined as:

$$
D_{H}^{\star}=\frac{D_{H}}{\sqrt{\sigma / g \Delta \rho}}
$$

and the liquid viscosity number $N_{\mu_{l}}$ is:

$$
N_{\mu_{l}}=\frac{\mu_{l}}{\left(\sigma \rho_{l} \sqrt{\sigma / g \Delta \rho}\right)^{1 / 2}},
$$

To avoid numerical discontinuity, Eq. (34) shall be programmed as:

$$
k_{l b}=0.0019 \min \left(30, D_{H}^{\star}\right)^{0.809}\left(\frac{\rho_{g}}{\rho_{l}}\right)^{-0.157}\left(N_{\mu_{l}}\right)^{-0.562}
$$





\section{ANNULAR-MIST INTERFACIAL DRAG}

In previous studies [44], CTF greatly overpredicted the two-phase pressure drop in the Ris $\varnothing$ tests. The Ris $\varnothing$ tests involve measuring film thickness, film mass flow rate, and two-phase pressure drop in a boiling tube. Geometry included both annular and tube geometries, and tests were both diabatic and adiabatic. Full details of the facility can be found in Wurtz [43]. For this work, two-phase pressure-drop comparisons were the figure of merit.

Similar behavior was previously noted in Lane [22]. This prompted work documented in Salko et al. [33] to implement a new set of annular-mist closure models and transition criteria. As part of the work for this milestone, the existing CTF model was reviewed, and the annular-mist interfacial drag is calculated by one of two approaches, depending on whether the film is classified as stable or unstable (i.e., wavy). Although this is a reasonable distinction to make, the approach for calculating unstable film interfacial drag is questionable. The stable film interfacial drag is calculated by using the Wallis model [40], and unstable film interfacial drag is calculated by using the maximum of five times the Wallis model value or the value predicted by the Henstock and Hanratty model [16]. This unstable film model was also question by Lane [22] in the development of his model.

A study was done as part of this work in which the Wallis model was used for stable and unstable film interfacial drag. Results for the baseline CTF prediction of two-phase pressure drop, prediction using the Wallis model for both subregions, and prediction using the Lane model [22] are shown in Figure 10.

Just by removing the unstable film interfacial drag assumption, the pressure-drop predictions improve considerably and are essentially in line with what was obtained with the Lane model. The Lane model, in addition to deriving a new set of interfacial drag models for annular-mist flow, also uses new annular-mist transition criteria, which leads to better predictions of the flow regime. In the case of Figures 10a and 10b, all flow regimes are identified as churn-turbulent, which is incorrect. As previously noted, future work is needed to introduce a more accurate annular-mist transition criteria to address this issue. For the remainder of the assessments performed in this report, the unstable film correction tested here will be used. 


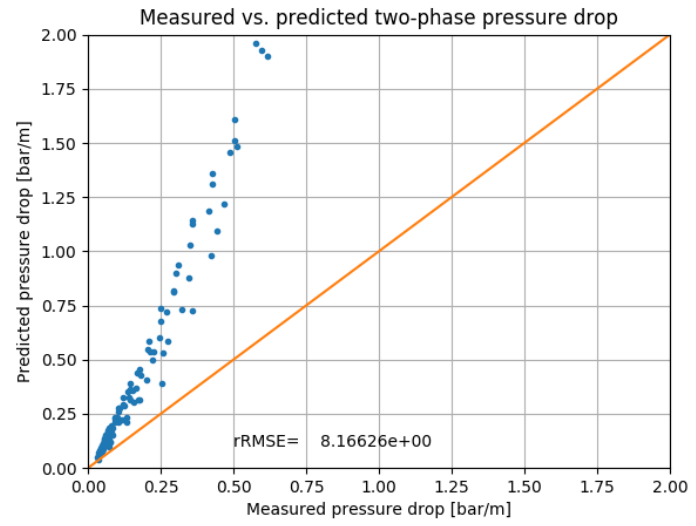

(a) Original model

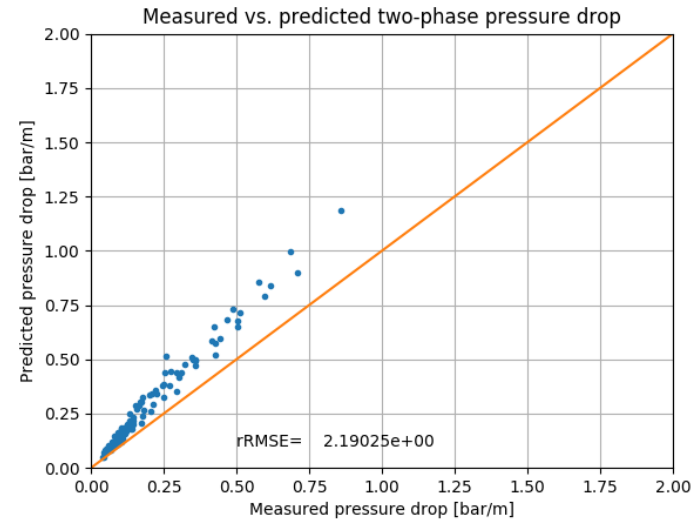

(b) Unstable film using Wallis model

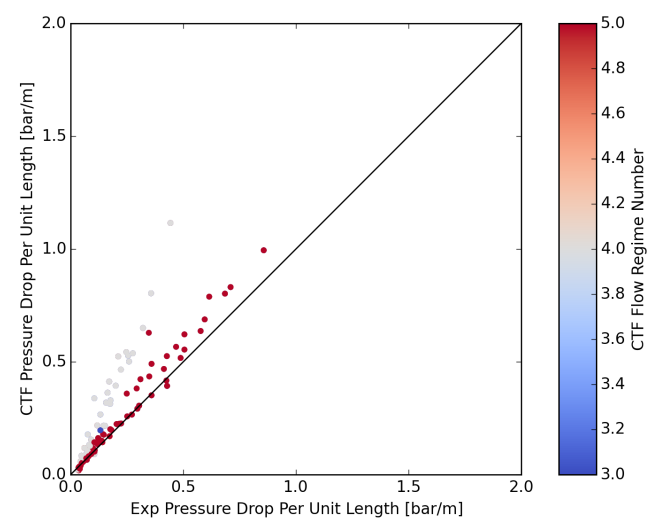

(c) Lane model. Color bar shows the flow regime, with 4 being the churn-turbulent regime and 5 being the annular-mist regime.

Figure 10. CTF prediction of two-phase pressure drop in the Risø facility by using (a) the base model, (b) the unstable film via the Wallis model for interfacial drag, and (c) the Lane model. 


\section{ASSESSMENT}

After completing modifications to the interfacial drag and subcooled boiling closure models, an assessment was done for tests in the CTF validation matrix, which can be used to understand the impact on the predicted void, wall temperature, and pressure drop. Each of the following sections covers a different category of tests in the matrix. Generally, for each category of tests, the baseline results are shown via the original CTF closure models, the results that use the drift-flux model are shown, and the results that use the drift-flux and ONB models combined are shown.

\subsection{Bartolomei}

Figures 11a-11c show the measured vs. predicted void fraction for all Bartolomei tests included in the validation matrix. CTF predictions of the Bartolomei tests exhibit a different behavior than the PSBT and BFBT tests, which were consistently overpredicted by CTF when it came to void fraction. Adding the drift-flux model reduces void prediction, and adding ONB reduces predicted void more markedly. This observation is consistent with the behavior in other two-phase tests that will be shown in the following sections and is consistent with expectations. For the Bartolomei cases, the general statistics are made slightly worse since most void measurements are in the low void region and are underpredicted by CTF to start. Recall from Section 2.1.1 that the underpredicted void cases are low-mass flux cases. Higher void regions are actually better predicted. 


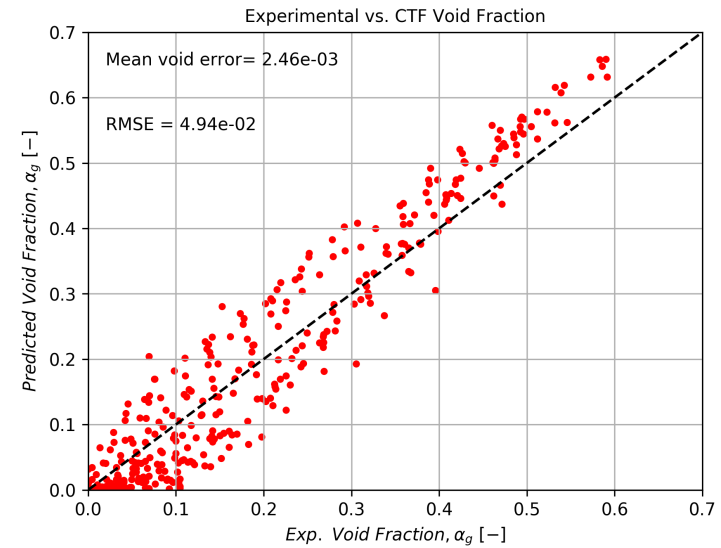

(a) Original model

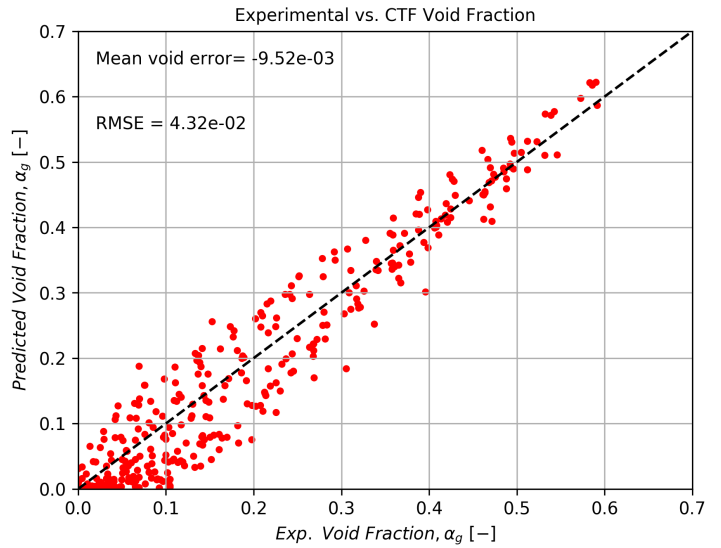

(b) Drift-flux model

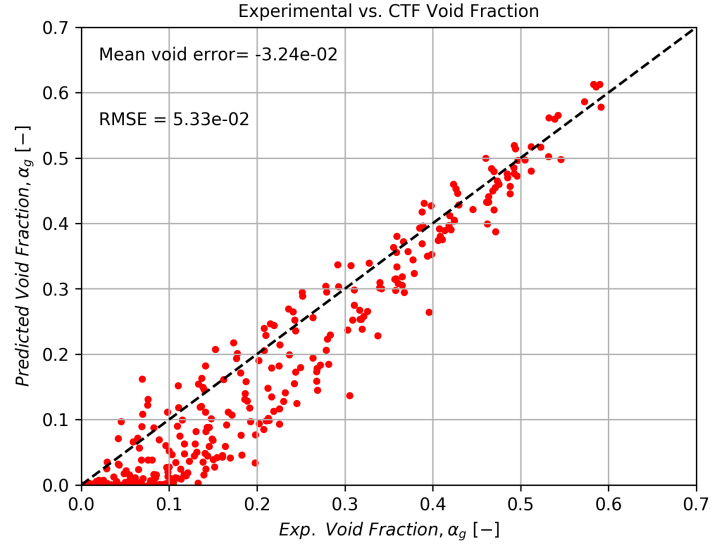

(c) Drift-flux model and ONB model

Figure 11. CTF prediction of void fraction in Bartolomei cases by using original models, drift-flux, and drift-flux/ONB models. 


\subsection{PSBT Single Channel}

The PSBT single-channel tests include void measurements made in four separate testing sections, each representing one subchannel geometry encountered in PWR geometry. Conditions are set so that significant voiding is encountered in the experiments. Full details on these experiments are found in Rubin et al. [30].

For comparison, all PSBT experimental data and CTF predictions from all four channel geometry types were condensed into one plot, as shown in Figure 12. In the figure, void prediction results for baseline, drift-flux, and drift-flux and ONB models are presented. The comparison statistics, mean error, and RMSE are also shown in the upper left. As indicated, CTF overpredicted void by $4.6 \%$ by using the baseline model. This was dropped to $1.5 \%$ by adding the drift-flux model and dropped further to $-0.8 \%$ by adding the ONB model to the drift-flux model. The RMSE is also slightly improved. As indicated, the drift-flux model tends to have the largest impact for higher void locations, and the ONB model tends to have the largest impact in lower void locations.

A benchmark study between CTF and VIPRE-1 was performed before this work and is documented in Zhao et al. [45]. The PSBT single-channel tests were included in this study. The VIPRE-1 model was set up to use the EPRI drift flux model to calculate phase slip, and subcooled steam quality was calculated by using a profile-fit model. In addition to VIPRE-01, CTF was also compared with the F-COBRA-TF results that were extracted from the PSBT benchmark report [24]. Figure 13 summarizes the comparison of the CTF results by using the original closure models, drift-flux, and drift-flux combined with ONB.

Additionally, the VIPRE-01 and F-COBRA-TF results are shown next to the CTF results. The mean errors for each code are shown for each geometry type. As indicated by the figure, the baseline CTF results were overpredicting void by a considerable margin and were significantly different than other popular $\mathrm{T} / \mathrm{H}$ codes used in the nuclear industry. Including the new closure models has helped significantly reduce the CTF bias and bring its void estimation in line with other popular $\mathrm{T} / \mathrm{H}$ codes. 


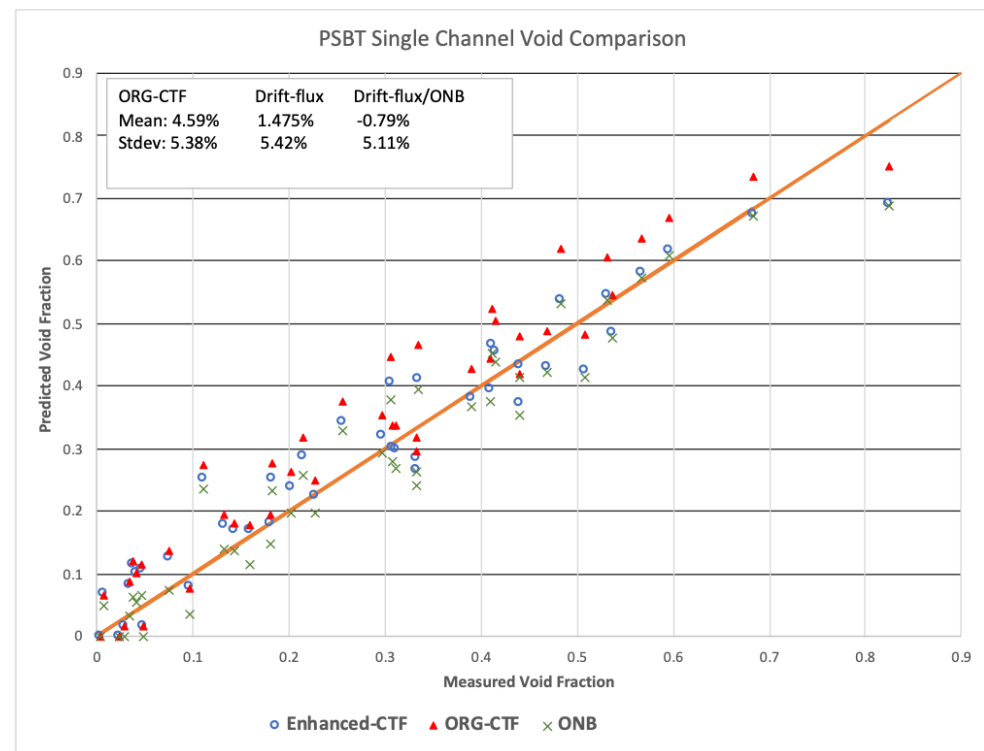

Figure 12. Validation of CTF to PSBT single-channel tests (Series 1-4) by using baseline models, drift-flux models, and ONB models.

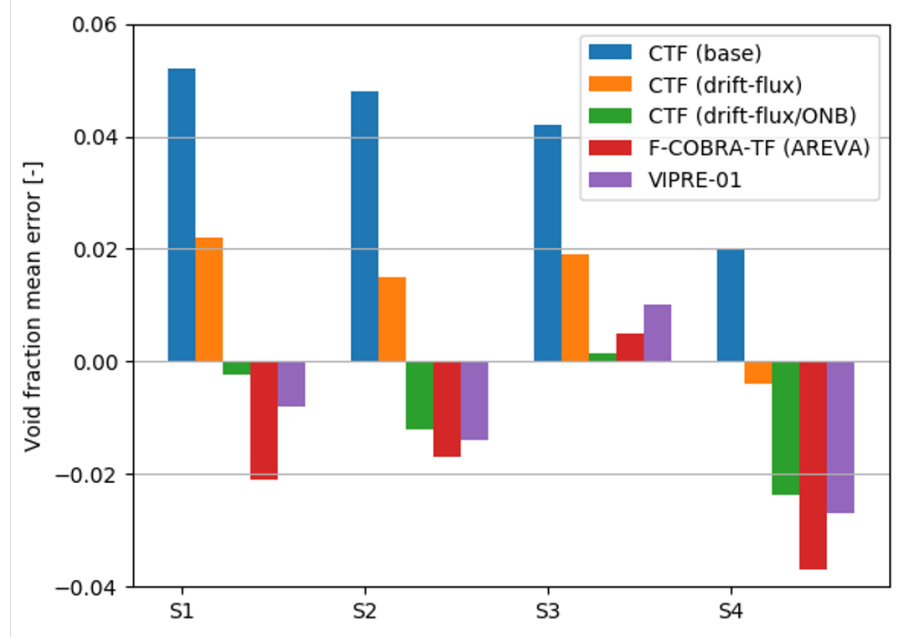

Figure 13. Comparison of CTF and VIPRE-01 mean error for PSBT single-channel tests. CTF results include the baseline models, drift-flux model, and drift-flux combined with ONB model. 


\subsection{PSBT Bundle Tests}

The PSBT bundle tests are $5 \times 5$ rod bundles representative of PWR geometry. Like the PSBT single-channel tests, the operating conditions are set so that significant voiding is encountered in the experiments. Void was measured for the center four subchannels of the bundle at three axial locations by using an x-ray densitometer. Full details of the experiments are available in Rubin et al. [30].

Results for baseline, drift-flux, and drift-flux/ONB models are shown for all three geometry types in Figures 14a, 14b, and 14c.

Pulling VIPRE-01 results from Zhao et al. [45], the comparison of CTF and VIPRE-01 are shown for the same three test series in Figures 15a, 15b, and 15c. 


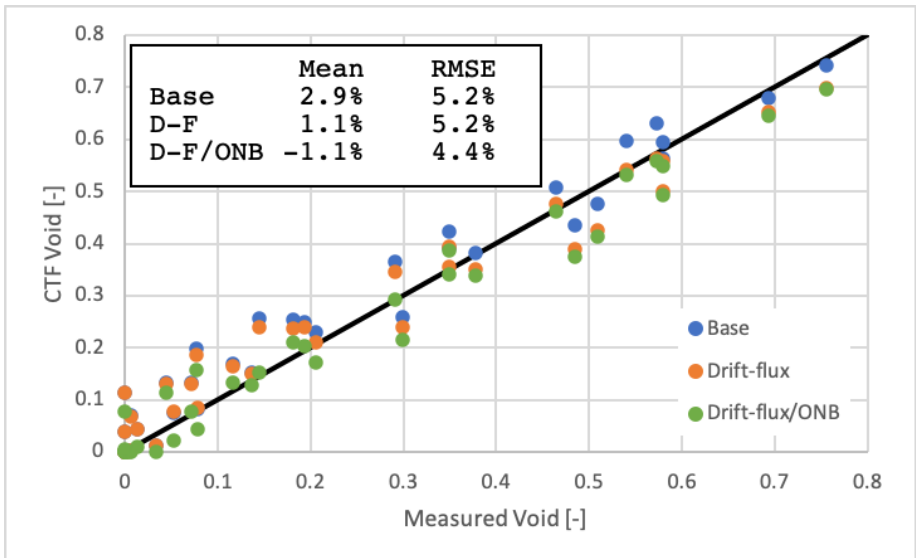

(a) PSBT Series 5 results.

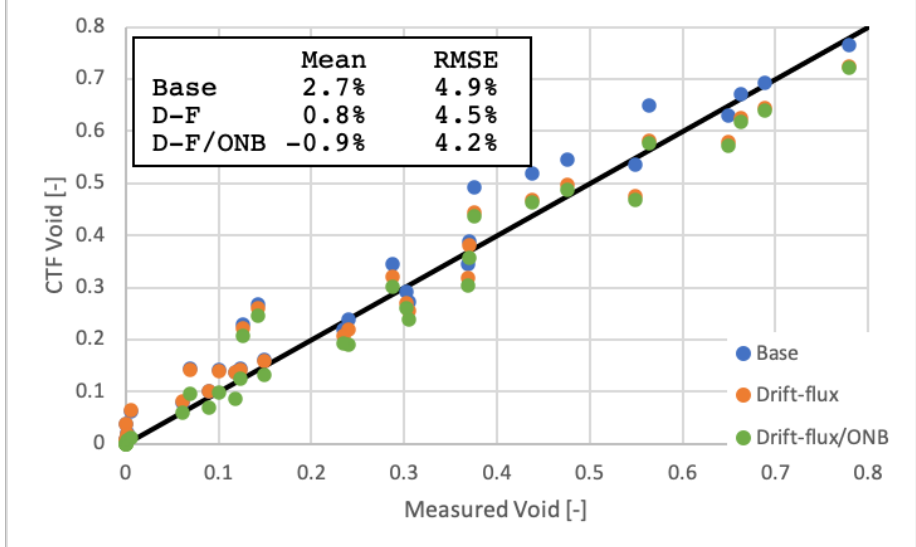

(b) PSBT Series 6 results.

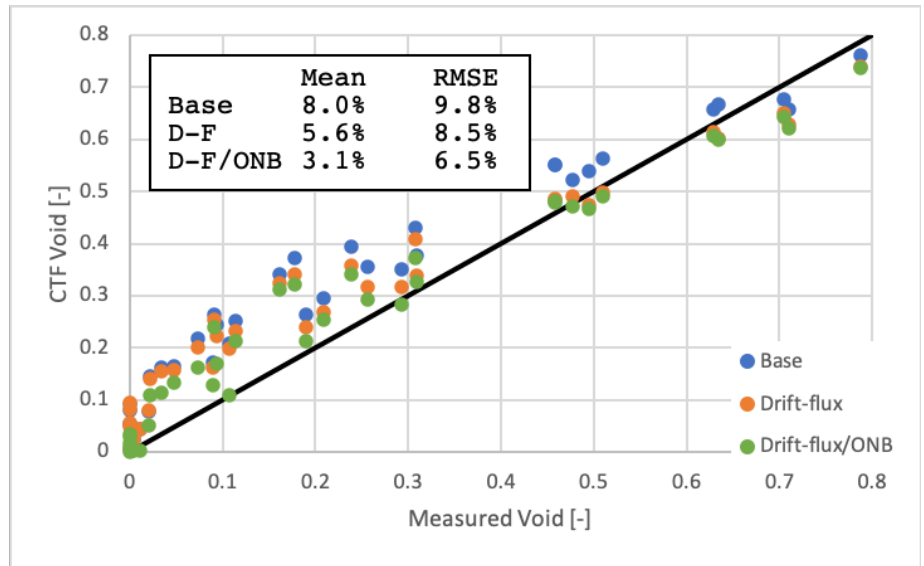

(c) PSBT Series 7 results.

Figure 14. PSBT bundle test results using CTF baseline, drift-flux, and drift-flux/ONB models. 


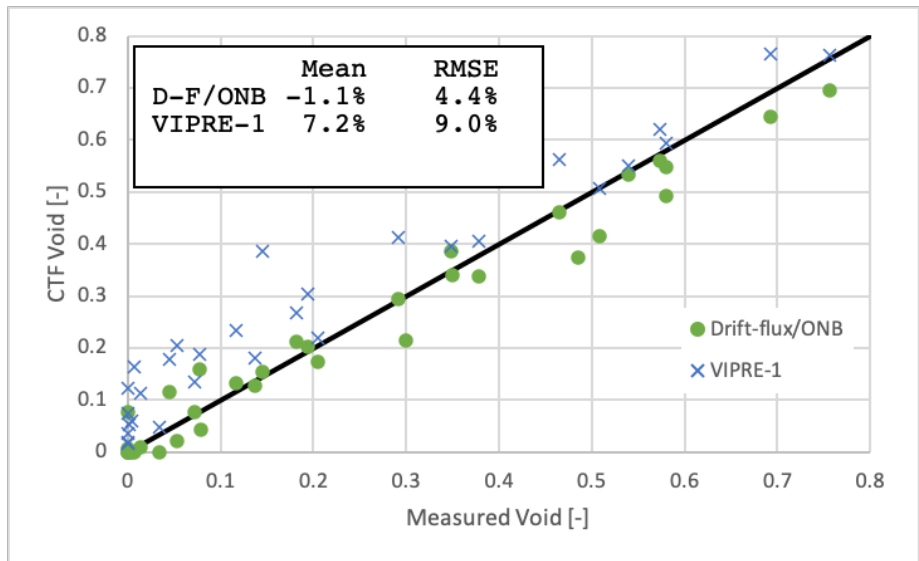

(a) PSBT Series 5 results.

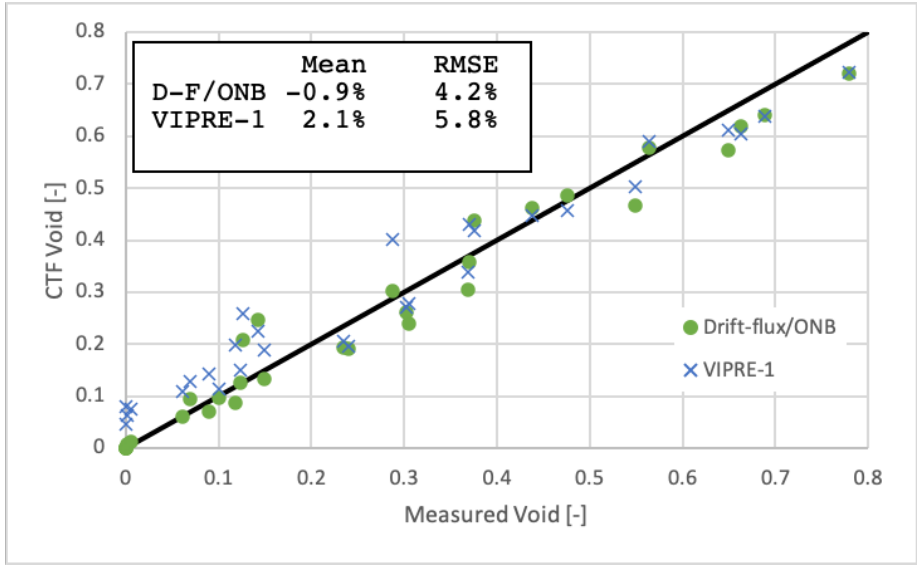

(b) PSBT Series 6 results.

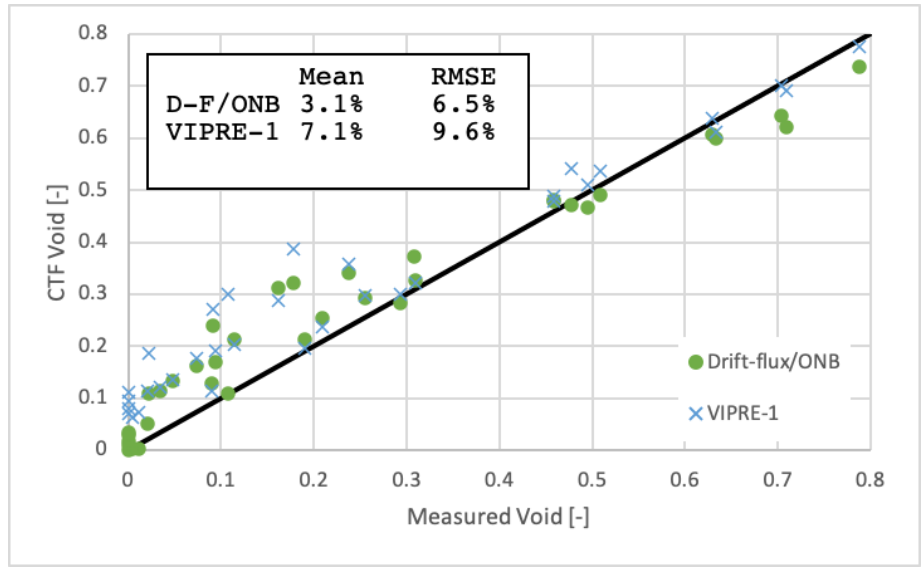

(c) PSBT Series 7 results.

Figure 15. Benchmark of CTF and VIPRE-01 for PSBT bundle tests using drift-flux and ONB model in CTF. 


\subsection{BFBT Bundle Void Distribution}

The BFBT bundle tests were performed on $8 \times 8$ rod bundles representative of BWR geometry with one bundle containing a large water rod. Different power shape configurations were used in the experiments, and a high-resolution computed tomography scan of the outlet void distribution was made in the steady-state tests. A full description of the assembly geometries and test conditions are given in Neykov et al. [23].

Figure 16 shows the comparison of the average outlet void for each bundle type that was tested by using the original models, drift-flux, and drift-flux with ONB. The overprediction at the $40 \%$ level was essentially eliminated by switching to the drift flux model. The void at the $80 \%$ level, which is mostly annular-mist flow, is essentially unchanged, and the void at the $60 \%$ level, which is churn-turbulent, is slightly worse. The churn-turbulent regime is a linear combination of interfacial drag values calculated for the annular-mist flow regime and the slug flow regime. In the current CTF flow regime map, the churn-turbulent regime starts at 50\% void and ends at the annular-mist transition point, which is at least $80 \%$. It is questionable whether an interpolation region that spans $30 \%$ void in the flow regime map is an appropriate assumption. Likely, it should span a smaller region. Furthermore, the annular-mist transition criteria currently implemented in CTF transitions at too large of a void fraction, sometimes much higher than $80 \%$ [22], which is not realistic. Future work will be required to fix this transition criteria and churn-turbulent boundaries in the flow regime map. 


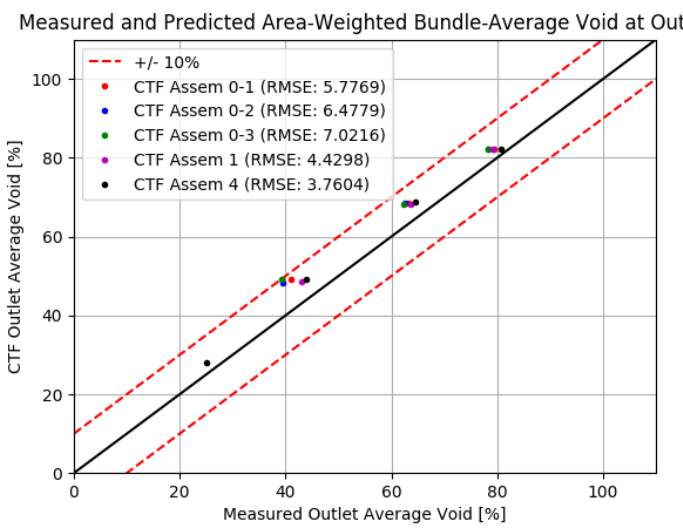

(a) Original model.

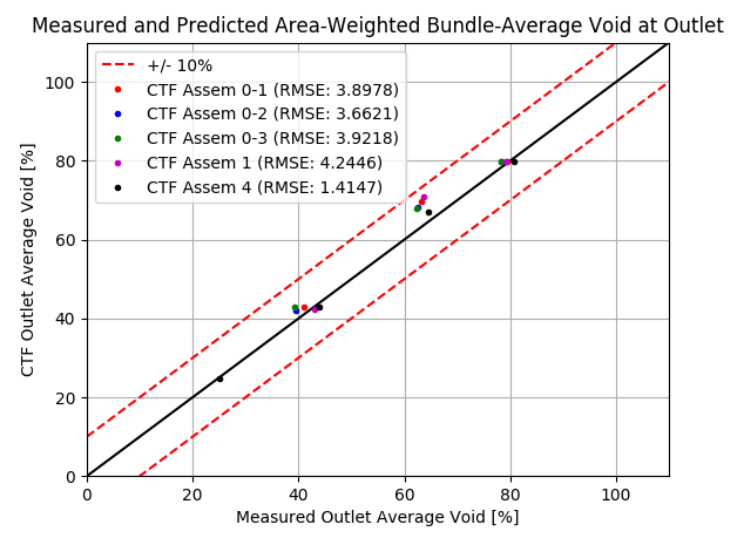

(b) Drift-flux model.

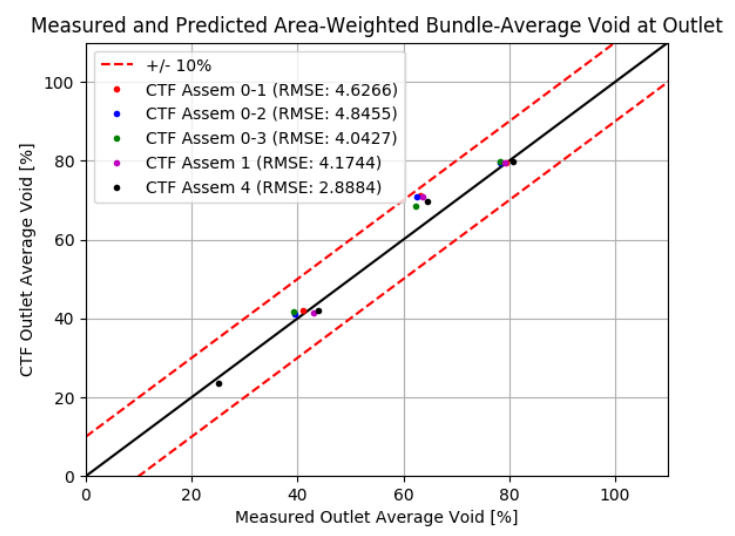

(c) Drift-flux and ONB model.

Figure 16. Comparison of CTF vs. experimental area-weighted bundle-averaged void fractions at outlet for the BFBT tests by using original, drift-flux, and drift-flux/ONB models. 


\subsection{Risø}

After correcting the film interfacial drag model discussed in Section 5., the effect of the drift flux model and ONB models were assessed. Figure 17a shows the results of applying the base drift-flux model for the Ris $\varnothing$ tests. This clearly worsens the pressure-drop prediction. Further analysis of the results revealed that the model is driving the two phases to the same velocity, essentially resulting in a slip ratio of 1.0. Because operating conditions for these tests are consistent with other validation tests that were tested and exhibited the opposite behavior, it was assumed that something about the geometry is not compatible with the drift flux model. In addition to the general form of the distribution parameter, which was presented in Eq. (21), Ishii also presented a form for tube geometry [17], which is shown as follows:

$$
C_{0}=1.2-0.2 \sqrt{\rho_{v} / \rho_{l}} \text {. }
$$

This model was also implemented into CTF. When switching to this model, the two-phase pressure-drop prediction behavior returns to the improvement that was realized with the interfacial drag correction. Using this form of the model and the ONB model results in the prediction shown in Figure 17b. Comparing this with Figure 10b shows that the drift-flux model and ONB model have very limited effect on the two-phase pressure-drop prediction. In the case of the high voids experienced in the Ris $\varnothing$ tests, the annular-mist interfacial drag and two-phase wall drag will dominate. 


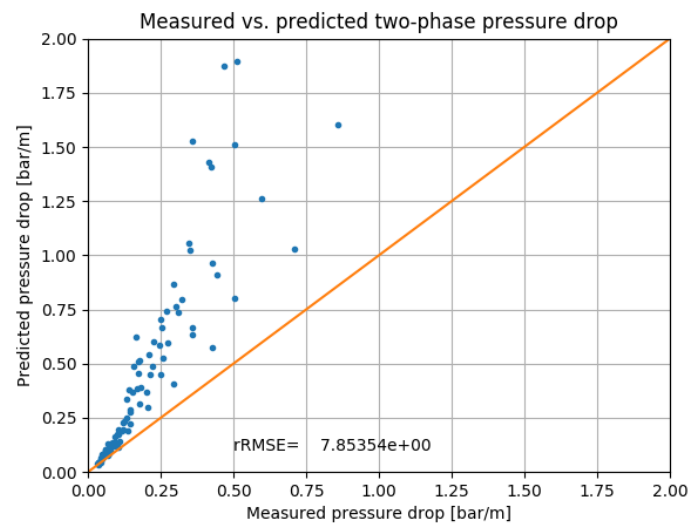

(a) Base drift-flux model.

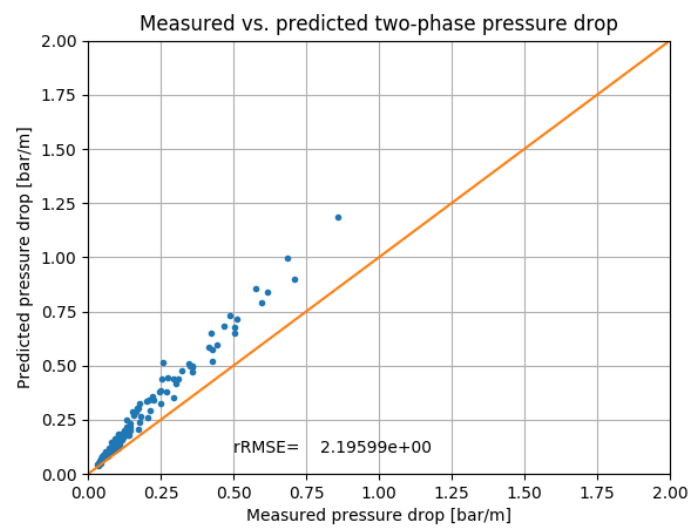

(b) Drift-flux model using tube distribution parameter and ONB model.

Figure 17. CTF prediction of two-phase pressure drop in Risø facility using the drift-flux model and the drift-flux model with tube distribution parameter and ONB model. 


\subsection{NESTOR Tests}

The NESTOR tests were used for testing the effect of the new ONB model on rod surface temperature predictions. Only boiling tests were considered for assessing the subcooled boiling model. Temperature measurements were made at multiple azimuthal locations and multiple axial locations in four different spans of the heated NESTOR facility. Because CTF only models four azimuthal surfaces per axial level of the rod, it was necessary to perform an interpolation of the CTF results to the measurement locations available in the NESTOR results. A nearest-neighbor interpolation scheme was used to prevent introducing "false fidelity" into the CTF results. An RMSE was calculated for each rod/grid span/test combination. To visualize these results, the RMSEs are plotted in a histogram, with the error being the horizontal access. One histogram is produced for each span so that the error being shown includes the RMSEs for all rods in that span for all tests run. These results are shown for Spans 1a, 1b, 2a, and 2b in Figures 18-21.

Two distributions are shown in the plots: the blue one represents the base model, and the translucent orange one shows the results via the ONB model. The ONB model clearly has little impact on Spans 1a and 2a since these spans are immediately downstream of mixing vane grids, which cause a large amount of turbulence-driven heat transfer enhancement. This will increase heat transfer and suppress boiling. CTF includes a grid spacer heat transfer enhancement model that captures this effect. Ultimately, the heat transfer behavior downstream of the mixing vane grids is mostly single-phase and thus will have impact to changes in the boiling heat transfer model.

However, Spans $1 b$ and $2 b$ are downstream of simple support grids, which have less of an impact on the heat transfer enhancement, meaning that boiling will be more prevalent. In both cases, the prediction error significantly shifts to smaller values when the ONB model is used over Thom. For Span 2b, which will have the most boiling due to being higher in the assembly, the improvement is most significant.

The subcooled boiling model was implemented into CTF with the primary intention of addressing void-prediction issues for subcooled flow, but these results demonstrate that it also favorably impacts the prediction of rod surface temperature in boiling tests. 


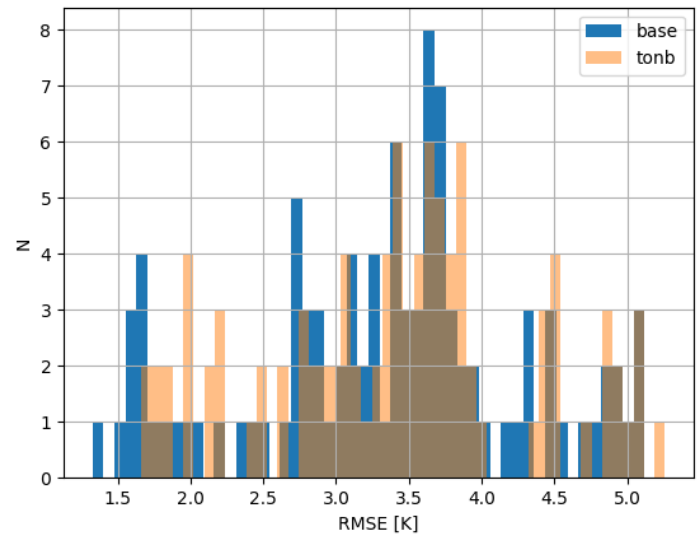

Figure 18. Comparison of spread of error between CTF predictions and NESTOR experimental results for surface temperature in Span 1a of the heated test facility when using the CTF Thom model vs. the new ONB model.

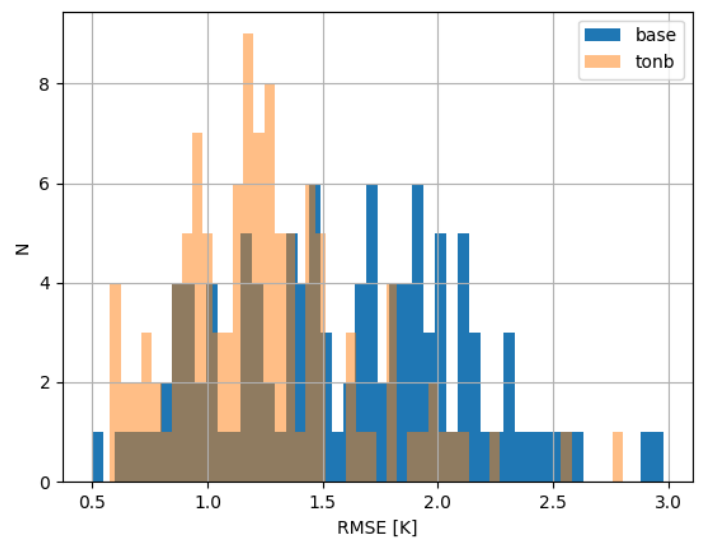

Figure 19. Comparison of spread of error between CTF predictions and NESTOR experimental results for surface temperature in Span 1b of the heated test facility when using the CTF Thom model vs. the new ONB model. 


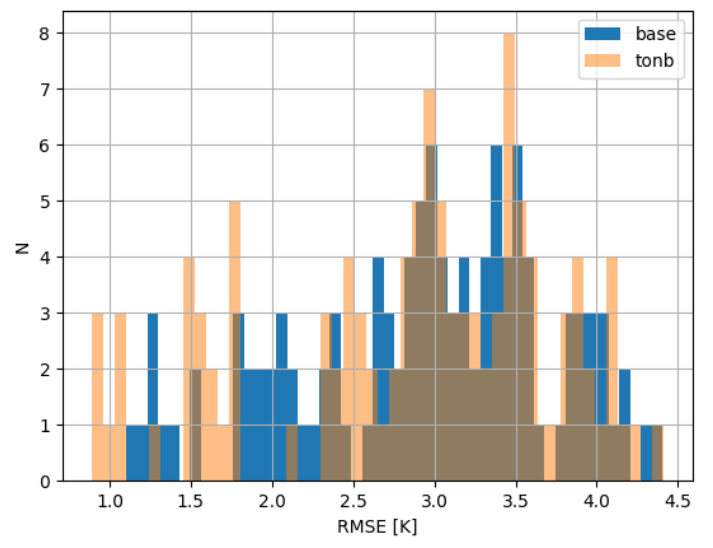

Figure 20. Comparison of spread of error between CTF predictions and NESTOR experimental results for surface temperature in Span 2a of the heated test facility when using the CTF Thom model vs. the new ONB model.

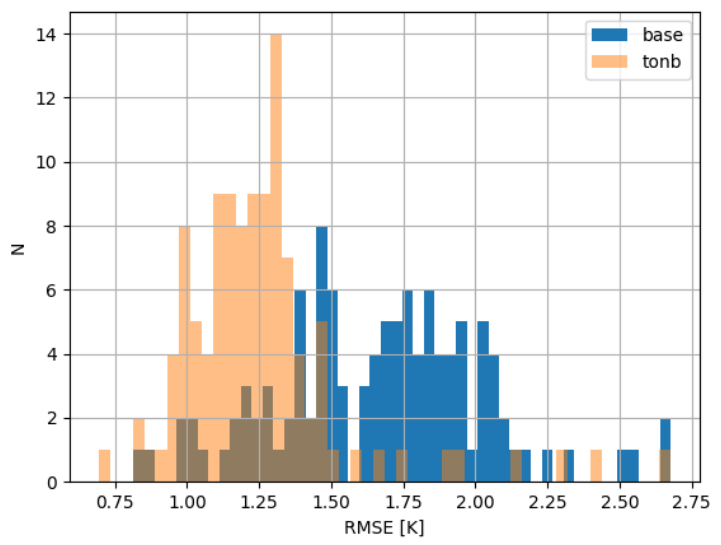

Figure 21. Comparison of spread of error between CTF predictions and NESTOR experimental results for surface temperature in Span $2 b$ of the heated test facility when using the CTF Thom model vs. the new ONB model. 


\subsection{Rohsenow Tests}

The Rohsenow tests were boiling tests performed in a $0.46 \mathrm{~cm}$ heated tube with a length of $23.9 \mathrm{~cm}$. Temperature measurements were made by seven thermocouples placed at different axial locations along the outside of the test section. Temperature measurement error was estimated to be $1.7 \mathrm{~K}$. Operating pressure varied between roughly 100 and $140 \mathrm{bar}$, inlet temperatures were between roughly 150 and $226^{\circ} \mathrm{C}$, inlet mass flux was between 2,600 and $7,900 \mathrm{~kg} / \mathrm{m}^{2} \mathrm{~s}$, and linear heat rate was between 65 and $136 \mathrm{~kW} / \mathrm{m}$. Full details of the facility are available in Rohsenow and Clark [29]. The wall temperature predictions are shown with the default and the new boiling model for the tests in Figure 22. The mean error in this case is actually slightly degraded when switching to the ONB model, but the scatter in the data is considerably improved. A greater deal of points are underpredicted with the base model, which helps bring the mean prediction error lower. 


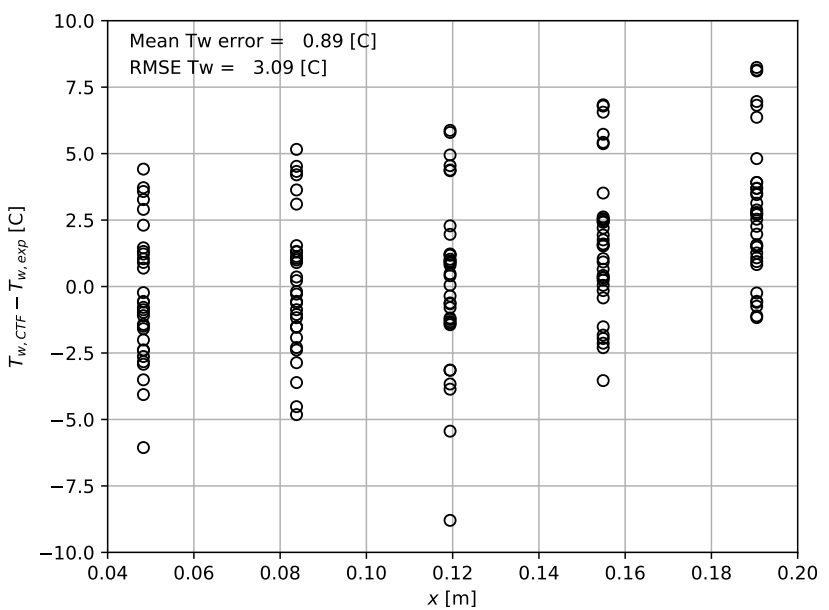

(a) Original model.

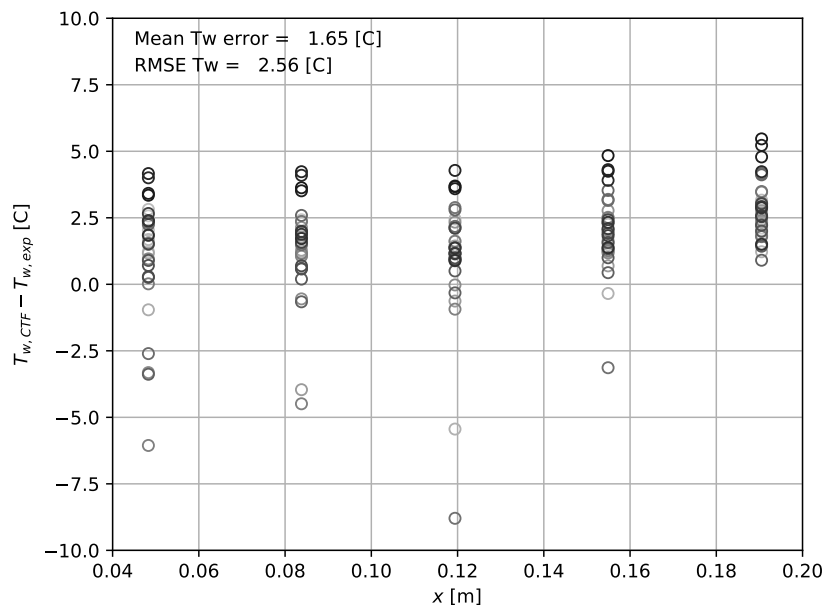

(b) ONB model.

Figure 22. Difference between CTF and experimental wall temperatures for the Rohsenow experiments.

\subsection{WALT Tests}

The WALT facility was a single-rod facility used to test heat transfer behavior during crud growth on a fuel rod. The tests include clean rod experiments at boiling conditions, so it is useful for testing of the heat transfer model. The annulus facility is $46 \mathrm{~cm}$ long and has a flow area of $0.6 \mathrm{~m}^{2}$. Four thermocouples are placed at different azimuthal locations inside the heater tube at the same axial location. Full details of the facility are found in Deshon [9]. The results of temperature predictions obtained via the Thom and ONB model are shown in Figure 23. The different datasets correspond to different experimental studies that were performed. As indicated, two of the tests experienced very high levels of error, regardless of the heat transfer model employed. These tests correspond to low-temperature tests. The outer temperatures had to be estimated from the inner temperature measurements by using ZIRLO thermal properties, so there could be a large uncertainty in this estimation for this region. Disregarding these tests, the remaining tests show a clear trend that predictions are improved when switching to the ONB model. 


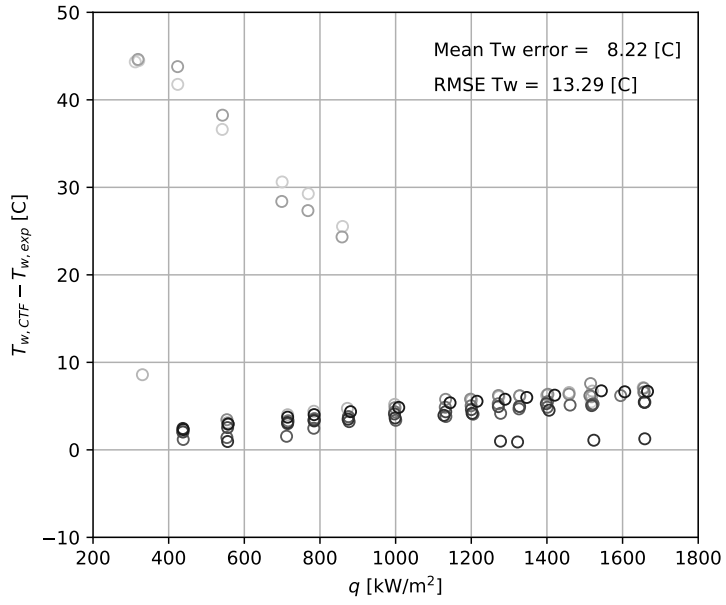

(a) Original model.

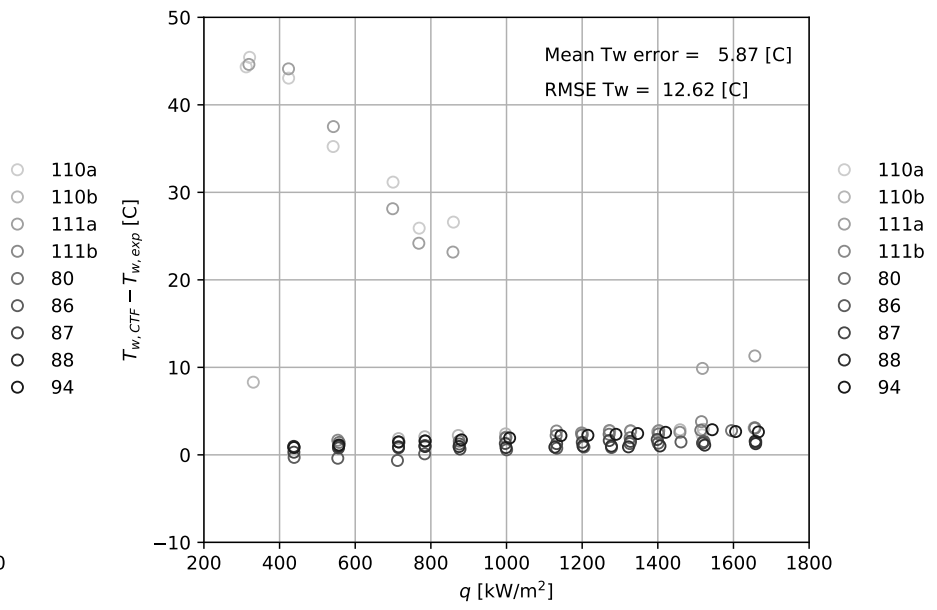

(b) ONB model.

Figure 23. Difference between CTF and experimental wall temperatures vs. heater flux for the WALT tests 



\section{CLOSURE MODEL CALIBRATION}

\subsection{Bayesian Calibration Setup}

Applying Bayesian inference to calibration problems provides both the optimal parameter values and the uncertainty associated with each free parameter, given the experimental data. Even for cases in which a traditional optimization approach encounters difficulties, such as when the RMS response surface is non-smooth near the optimal value, a numerical approach that uses Markov-chain Monte Carlo (MCMC) might still provide accurate mean and higher moments of the parameter distributions at the expense of thousands of model evaluations. For quickly executing models, this computational expense is not a concern.

Let the model predictions be denoted by $\left.y_{m}\right|_{\theta}$, where $\boldsymbol{\theta}$ represents a set of model parameters, such as the unknown leading coefficients in a closure model. There could be several unknown model parameters so that $\boldsymbol{\theta}$ denotes a vector. Let the experimental dataset be denoted $y_{d}$ and the set of all available data be $\left\{y_{0, d}, \ldots y_{N, d}\right\}$, where there are $N$ data points available for calibration. The residual is defined by the difference between the model predictions and the experimental data, $r_{i}=\left.y_{m}\right|_{\theta}-y_{i, d}$. The sum of squares error is defined by $S S E=\sum_{i}^{N}\left(r_{i}\right)^{2}$.

Assuming that the residuals of the experimentally observed values about the model predictions follow a Gaussian-like distribution, $r \sim \mathcal{N}\left(0, \theta_{\sigma}\right)$, with unknown standard deviation $\theta_{\sigma}$, the likelihood of the data given a parameter set $\boldsymbol{\theta}$ was taken to be of the form shown in Eq. (39). In this case with limited experimental data, $y_{d}=\left\{y_{0}, y_{1}, \ldots y_{N}\right\}_{d}$, with $N$ being the number of experimental data points, the likelihood is given by Eq. (40):

$$
\pi\left(y_{d} \mid \boldsymbol{\theta}\right)=\frac{1}{\left(2 \pi \theta_{\sigma}^{2}\right)^{N / 2}} \exp \left(-\frac{S S E}{2 \theta_{\sigma}^{2}}\right) .
$$

Assuming that the data-model residuals are independent and identically distributed, the likelihood for all data given a model with parameter set, $\boldsymbol{\theta}$, can be expressed by Eq. (40):

$$
\pi\left(\left\{y_{0, d}, \ldots y_{N, d}\right\} \mid \boldsymbol{\theta}\right)=\prod_{i}^{N} \frac{1}{\left(2 \pi \theta_{\sigma}^{2}\right)^{1 / 2}} \exp \left(-\frac{\left(\left.y_{i, m}\right|_{\boldsymbol{\theta}}-y_{i, d}\right)^{2}}{2 \theta_{\sigma}^{2}}\right) .
$$

However, although Eq. (40) can be evaluated, the goal is to find the probability density of $\boldsymbol{\theta}$ given the data or $\pi\left(\theta \mid y_{d}\right)$. The problem can be rearranged slightly by recalling the product rule of probability:

$$
\pi\left(\boldsymbol{\theta} \mid y_{d}\right) p\left(y_{d}\right)=\pi\left(y_{d} \mid \boldsymbol{\theta}\right) p(\boldsymbol{\theta})
$$

or

$$
\pi\left(\boldsymbol{\theta} \mid y_{d}\right)=\pi\left(y_{d} \mid \boldsymbol{\theta}\right) p(\boldsymbol{\theta}) / p\left(y_{d}\right) .
$$

The denominator can be expressed as: $p(y)=\int p(y \mid \boldsymbol{\theta}) p(\boldsymbol{\theta}) d \boldsymbol{\theta}$. 


$$
\pi\left(\boldsymbol{\theta} \mid y_{d}\right)=\frac{\pi\left(y_{d} \mid \boldsymbol{\theta}\right) p(\boldsymbol{\theta})}{\int \pi\left(y_{d} \mid \boldsymbol{\theta}\right) p(\boldsymbol{\theta}) d \boldsymbol{\theta}} .
$$

The prior distribution, $p(\boldsymbol{\theta})$, is a free choice in the Bayesian approach. In principle, the prior can be any valid density function, but it should be carefully chosen to reflect the prior beliefs on the value for $\boldsymbol{\theta}$.

The probability density function (pdf) $\pi\left(\theta \mid y_{d}\right)$ is known as the posterior $p d f$. This is a properly normalized pdf that denotes the probability density of $\boldsymbol{\theta}$ given the available data $y_{d}$. Obtaining an estimate of this posterior pdf of the model parameters given the experimental data is the goal of the Bayesian model calibration process applied in this work.

\subsubsection{MCMC Implementation}

The computation of the integral in the denominator of Eq. (43) can be circumvented by employing an MCMC numerical method. When using MCMC, the target density function must only be known up to a constant— $\pi(\theta \mid y) \propto \pi(y \mid \theta) f(\theta)$ — to draw samples from it. The Differential Evolution Adaptive Metropolis (DREAM) MCMC algorithm was used in the following sections to draw samples from Eq. (43). DREAM was selected because it is robust to multimodal distributions, automatically adapts to the scale of the posterior without having to specify parameters governing a proposal distribution, and is easily run in parallel.

Several candidate Bayesian inference software packages were considered to numerically evaluate Eq. (43). BiPyMc is a Python package developed to solve general inference problems, including model calibration problems. BiPyMc implements the DREAM MCMC algorithm [39] and other adaptive differential evolution-based MCMC algorithms. An additional capability to perform Bayesian surrogate surface construction via Gaussian processes is also incorporated into this package to solve optimization problems when MCMC is too costly. Additionally, a second Python package called emcee [11] was also investigated as part of this work. Emcee implements the stretch-move ensemble MCMC algorithm, which can be executed on multiple processors in parallel, but requires a larger number of chains when compared with DREAM.

The differential evolution-based Markov chain Monte Carlo (DE-MCMC) method was developed by Braak [37] and later extended by Vrugt et al. by adding an adaptive subspace sampling strategy to boost performance in high-dimensional problems, resulting in the DREAM algorithm [39]. The primary advantage of DREAM over the common Metropolis-Hastings algorithm is that it does not need to explicitly form a proposal density distribution. In Differential evolution based Markov chain Monte Carlo (DE-MCMC), proposals are generated from the current state of ensemble of chains without the need to pre-specify a parametric proposal distribution function, as would be required in a Metropolis-Hastings approach. Each step of the DE-MCMC method is provided in algorithm 1. Fully parallel implementations of DE-MCMC and DREAM samplers are available in the Python programming language for use in this work. 


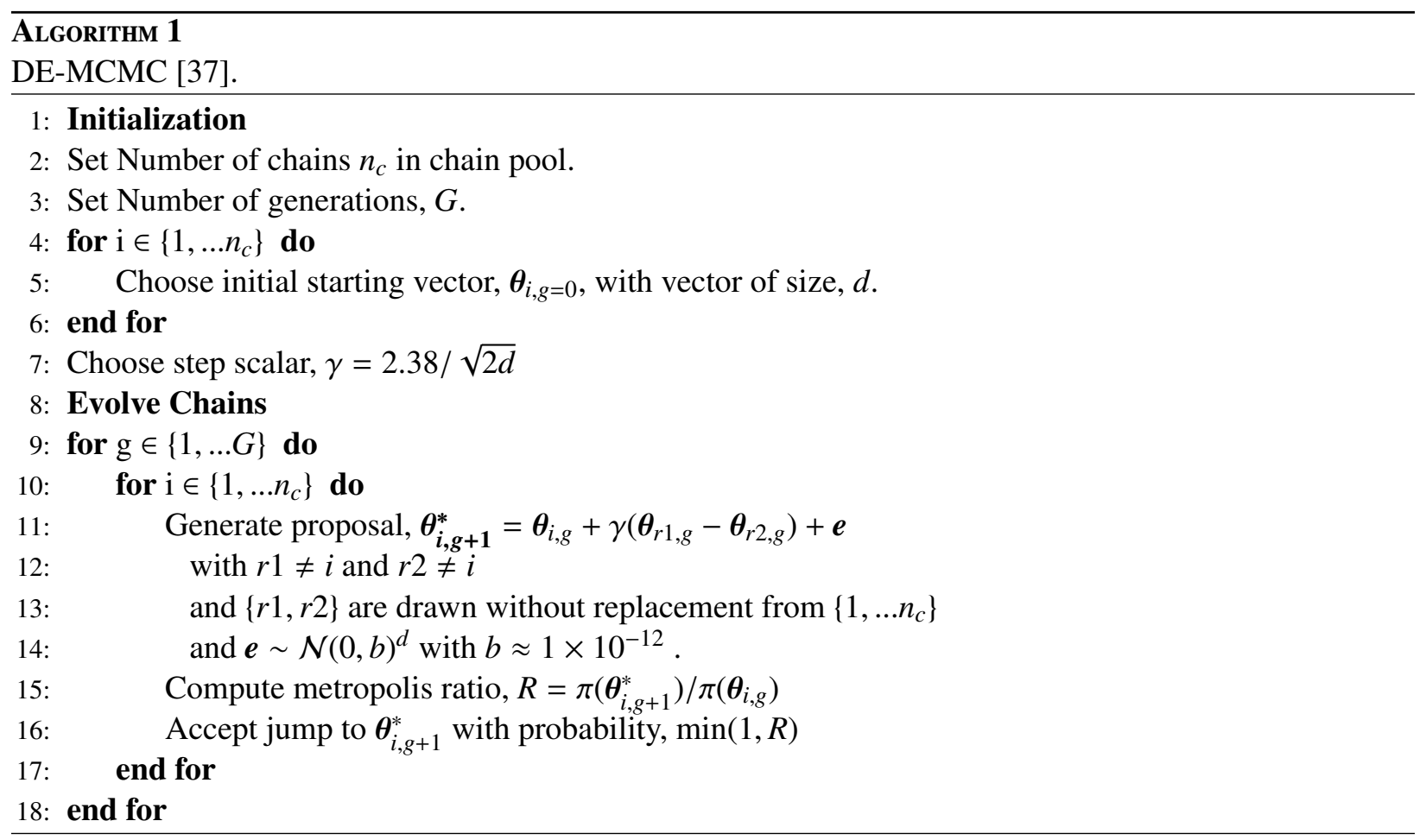

\subsection{Calibration Parameter Selection and Development of Surrogate Models}

The calibration parameters were down-selected from the work of Wu et al. [42] in which an inverse uncertainty quantification (UQ) analysis was performed for the TRACE code. In terms of multiplication factors, the list of parameters selected in $\mathrm{Wu}$ et al. [42] based on a sensitivity analysis included the single-phase liquid-to-wall heat transfer coefficient, subcooled boiling heat transfer coefficient, wall drag coefficient, interfacial drag (bubbly/slug rod bundle) coefficient, and interfacial drag (bubbly/slug vessel) coefficient. From this list of coefficients, the single phase coefficient was discarded since the focus of the current study is on two-phase flows, which also informs the experimental data selected for calibration. The default CTF model does not distinguish between interfacial drag coefficients for different geometries since the interfacial drag models in TRACE do; thus, no distinction was made in the interfacial drag multipliers used in CTF. To this list, a multiplier for the liquid film interfacial drag coefficient in annular flow was added to ensure that high void fraction regimes are also covered. Rather than use a single multiplier on the wall drag, the parameters of the two-phase multiplier model used in CTF were exposed and were calibrated instead so that the model is extensible to different thermal-hydraulic conditions and geometries. The two-phase multiplier model in CTF is of the form shown as follows:

$$
\Phi^{2}=A \alpha_{l}^{B}
$$

where the $\Phi^{2}$ parameter is the two-phase multiplier, $\alpha_{l}$ is the liquid void, and $A$ and $B$ are coefficients, which have default values of 1.0 and -2.0, respectively. The two-phase multiplier will be multiplied by the single-phase friction force to account for the two-phase enhancement of frictional losses. More details can be found in Salko et al. [34]. These parameters are represented by variable names k_xkwlxA and 
k_xkwlxB, respectively. The definition of the six chosen multipliers are given in Table 3 . The default value of all multipliers is 1.0 ,except $\mathrm{k} \_x k w l x B$, whose default value is -2.0 .

Table 3. Chosen calibration parameters.

\begin{tabular}{rlr}
\hline Multiplier & Description & Default \\
\hline k_xkwlxA & Two-phase wall drag coefficient A in $\Phi^{2}=A \alpha_{l}^{B}$ & 1.0 \\
k_xkwlxB & Two-phase wall drag coefficient B in $\Phi^{2}=A \alpha_{l}^{B}$ & -2.0 \\
k_xk_sb & Small bubble interfacial drag coefficient multiplier & 1.0 \\
k_xk_slg & Slug bubble interfacial drag coefficient multiplier & 1.0 \\
k_xk_anrflm & Annular-mist liquid film interfacial drag coefficient multiplier & 1.0 \\
k_hnb & Nucleate boiling heat transfer coefficient multiplier & 1.0 \\
\hline
\end{tabular}

The next step after selecting parameters to test was to select the tests that would be used for the calibration activity. Tests were selected from the CTF validation matrix since CTF models and data processing tools were already available. The focus for calibration is to select experimental datasets with void fraction and two-phase pressure-drop measurements. Initial selected tests included the PSBT single-channel tests, BFBT bundle void distribution tests, Ris $\emptyset$ tests, and Thom heat transfer tests. The PSBT single-channel tests are chosen to calibrate the interfacial drag coefficients in the small bubble and the slug flow regimes as the majority of the tests span those two flow regimes, whereas the BFBT (Assembly Type 1) and Ris $\varnothing 200$ series (unheated tests) tests are expected to be crucial for influencing the optimum value for the annular liquid film drag multiplier and two-phase multiplier parameters. The BFBT bundle tests included five different assembly types, which had different geometries and different power distributions. To prevent from having to include these parameters in the surrogate, only one assembly type was used to develop the surrogate, which was Assembly Type 1. Additionally, the Ris $\emptyset$ tests included several series of tests (i.e., heated and unheated, tubular and annular geometry). The unheated tests were chosen to prevent wall heat transfer effects from impacting the surrogate. Finally, the Thom tests were included for a similar reason; calibrating the boiling heat transfer term to match the void can result in the wall temperature prediction performing poorly, so wall temperature measurements are also needed.

Another reason for calibrating multiple datasets simultaneously is to prevent one solution term from being optimized while degrading the prediction of another related solution parameter. For example, interfacial drag will affect void fraction prediction, but it will also affect the two-phase pressure drop. Similarly, the boiling heat transfer can affect void prediction and wall temperature.

Once the parameters were selected, a preliminary sensitivity analysis was conducted, starting with the PSBT single-channel tests, which include experimental channel average void fraction measurements that are $1.4 \mathrm{~m}$ from the inlet. The results of the sensitivity analysis are shown for two multipliers (keeping the other multipliers as default values) for two extreme cases by using the base CTF model, which is defined later. Figure 24 shows the sensitivity of void prediction to k_hnb, and Figure 25 shows the sensitivity of void prediction to $\mathrm{k} \_\mathrm{xk} \_s b$.

The summary of the sensitivity analysis on the RMSE values is shown in Tables 4, 5, and 6, for the PSBT single-channel tests (Base RMSE [\%]: 5.796), BFBT bundle void distribution tests (Base RMSE [\%]: 7.447), and Ris $\varnothing$ tests (Base RMSE [bar/m] : 0.1314), respectively, for all six selected multipliers (five for adiabatic Ris $\varnothing 200$ series tests) for lower and upper bounds defined in Table 9. 


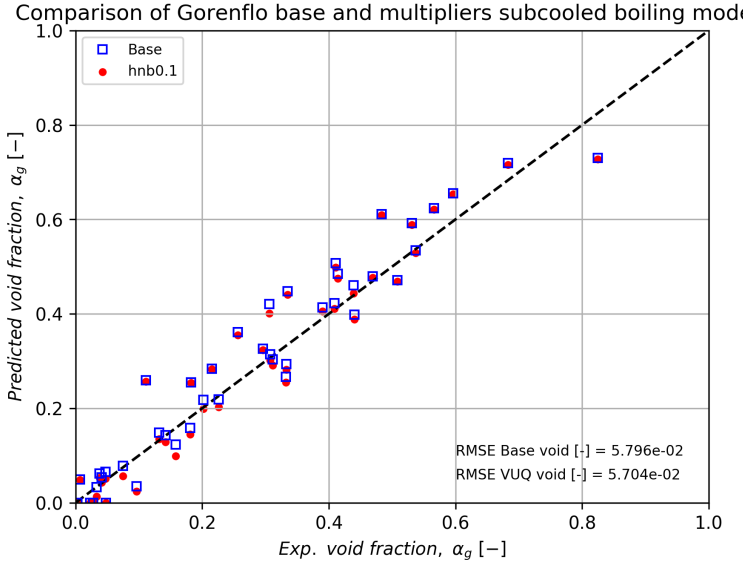

(a) $k \_h n b=0.1$

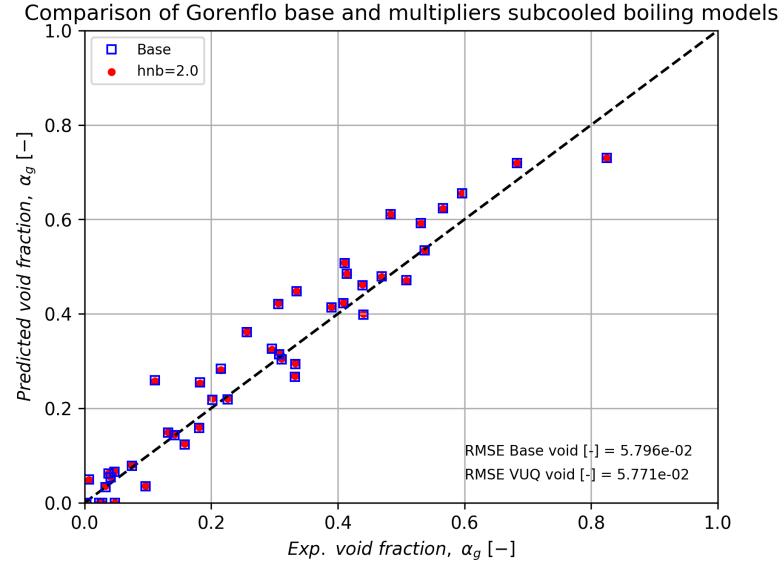

(b) $k \_h n b=2.0$

Figure 24. CTF sensitivity analysis in CTF void fraction prediction for the boiling heat transfer multiplier.

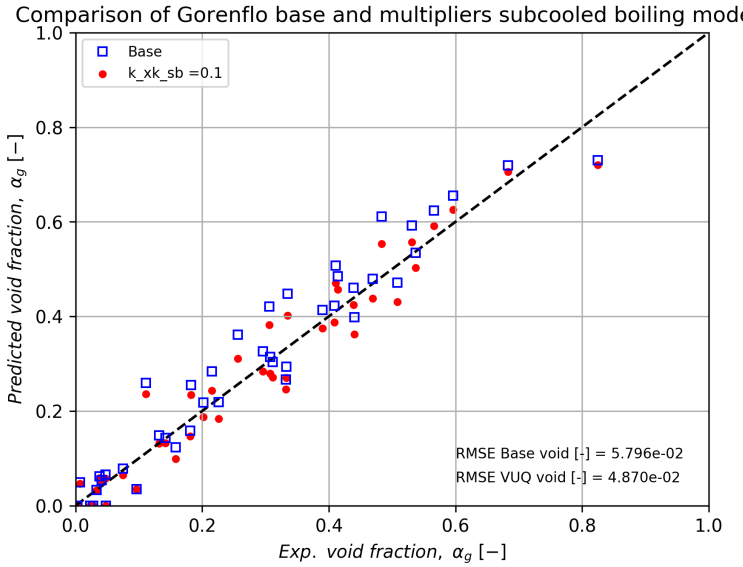

(a) $k \_x k \_s b=0.1$

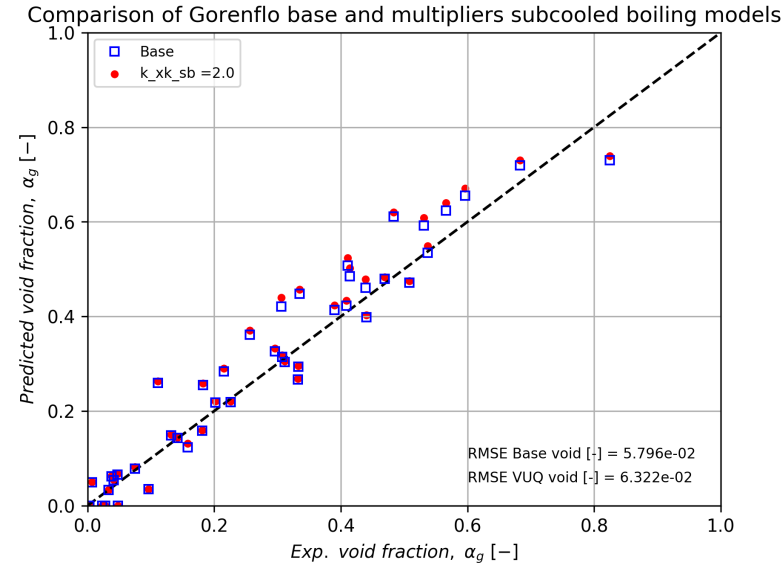

(b) $\mathbf{k} \_x k \_s b=2.0$

Figure 25. CTF sensitivity analysis in CTF void fraction prediction for the interfacial drag small bubble multiplier. 
Table 4. Sensitivity analysis of CTF RMSE (percent void fraction) values for the PSBT single-channel tests for the selected multipliers

\begin{tabular}{lccc}
\hline Multiplier name & Lower bound RMSE & Upper bound RMSE & $\Delta R M S E / \Delta k_{\text {mult }}$ \\
\hline k_xkwlxA & 6.424 & 5.520 & 0.476 \\
k_xkwlxB & 5.438 & 6.112 & -0.337 \\
k_xk_sb & 4.870 & 6.322 & -0.764 \\
k_xk_slg & 5.685 & 5.936 & -0.132 \\
k_xk_anrflm & 5.794 & 5.802 & -0.004 \\
k_hnb & 5.704 & 5.771 & -0.035 \\
\hline
\end{tabular}

Table 5. Sensitivity analysis of CTF RMSE (percent void fraction) values for the BFBT (all assembly types) channel tests for the selected multipliers.

\begin{tabular}{lccc}
\hline Multiplier name & Lower bound RMSE & Upper bound RMSE & $\Delta R M S E / \Delta k_{\text {mult }}$ \\
\hline k_xkwlxA & 9.005 & 6.863 & 1.127 \\
k_xkwlxB & 7.283 & 8.296 & -0.507 \\
k_xk_sb & 5.957 & 8.237 & -1.200 \\
k_xk_slg & 7.169 & 7.685 & -0.272 \\
k_xk_anrflm & 7.419 & 7.510 & -0.048 \\
k_hnb & 7.167 & 7.512 & -0.182 \\
\hline
\end{tabular}

From Table 4, it can be observed that the nucleate boiling heat transfer coefficient as defined in Table 3 is the least important parameter. Similar conclusions were obtained with other experimental datasets chosen for calibration. The PSBT single-channel tests are insensitive to the annular liquid film coefficient because very few cases in those tests reach voids high enough to enter annular-mist flow. Other tests, such as the Ris $\varnothing 200$ series (adiabatic) tests, are sensitive to the annular liquid film coefficient. Since the boiling heat transfer coefficient is fairly insensitive for all datasets except the Thom dataset, it was decided to simplify the calibration by dropping this multiplier and the Thom tests.

During the initial simulation of the test sets, it was decided that a CTF surrogate must be developed for each test set. The primary reason to use a surrogate model for replacing full CTF calculations in the calibration is to reduce computation times. A single evaluation of the likelihood function involves

Table 6. Sensitivity analysis of CTF RMSE (two-phase pressure gradient) values for the Risø tests for the selected multipliers.

\begin{tabular}{lccc}
\hline Multiplier name & Lower bound RMSE & Upper bound RMSE & $\Delta R M S E / \Delta k_{\text {mult }}$ \\
\hline k_xkwlxA & 0.120 & 0.212 & -0.048 \\
k_xkwlxB & 0.351 & 0.175 & 0.088 \\
k_xk_sb & 0.105 & 0.160 & -0.029 \\
k_xk_slg & 0.110 & 0.155 & -0.024 \\
k_xk_anrflm & 0.193 & 0.345 & -0.080 \\
\hline
\end{tabular}


executing CTF models for a variety of experimental setups—-here, the PSBT cases, Ris $\varnothing$ series, and BFBT cases-given a fixed set of closure model multipliers. To compute the likelihood, the CTF predictions are compared with the experimental data for each case to obtain a residual vector. The currently considered experimental suite constitutes 43 independent CTF simulations for the PSBT single-channel tests, 243 independent CTF simulations for the BFBT tests, and 81 independent CTF simulations for the Ris $\varnothing$ tests. If each experimental case were simulated via CTF, the run time of the Bayesian calibration would be too large to be feasible since the MCMC method requires a full likelihood function evaluation at each sample. Instead, the predictions of CTF must be replaced with a quickly executing surrogate model, which mimics CTF's response to boundary conditions, and with model parameters as closely as possible. To this end, a variety of surrogate construction techniques were investigated.

Prior work conducted by Wu et al. [42] showed that a Gaussian process regression strategy can be used to enable the Bayesian calibration of closure models in the T/H code TRACE. However, the experimental data considered in Wu et al. [42] were not as large or as diverse as the data included in the present work. A relatively large-scale model calibration performed by Collins and Gurecky [7] used gradient boosting to produce a neutronic-model surrogate, which enabled the Bayesian inference of parameters in a PWR coolant chemistry package. Gradient boosting is attractive due to its: (1) ease of training due to only containing a few hyperparameters, (2) fast training times compared with neural networks and for large datasets and Gaussian processes, (3) ability to scale to massive dataset sizes and high dimensionality, and (4) extremely fast model evaluation times. The primary disadvantage is the piecewise constant prediction that a gradient boosted model produces. A piecewise constant model struggles to capture smoothly the varying functions and essentially behaves as a nearest-neighbor model when extrapolated. In this regard, Gaussian processes, neural networks, and support vector machines are better suited to produce smooth and potentially continuously differentiable models.

\subsubsection{CTF Models}

The CTF modeling options used for the surrogate development and subsequent calibration included the ONB model discussed in Section 3. and the interfacial drag fix for annular mist flow discussed in Section 5. The original CTF interfacial drag models were used for small and large bubble flow since the drift-flux model was not ready for when the surrogate models were being generated. In future work, the calibration will be performed via the drift-flux model in place of the legacy CTF models.

\subsubsection{Gradient Boosting}

Gradient boosting is a supervised machine learning method that is applicable to both regression and classification problems. The modern gradient boosting algorithm was developed by Friedman et al. [12]. Generalized boosting development was significant because it reenvisioned previous boosting algorithms, such as AdaBoost, as special cases of gradient boosting with specific loss functions. Gradient boosting can be viewed as a numerical optimization procedure performed in function space to find a function $\mathcal{F}_{M}$ that maps arbitrary inputs, $\mathbf{p}$, to the expected outputs, $y$, where $\mathcal{F}_{M}$ is given by:

$$
\mathcal{F}_{M}=\operatorname{argmin}_{F} \underset{y, \boldsymbol{p}}{\mathbb{E}}(L(y, F(\boldsymbol{p})))
$$


where $L$ is a loss function and is taken to be the sum of squares error between the gradient-boosted model function $\mathcal{F}_{M}$ and the CTF predictions, $y$, in this work. The CTF predictions for a wide variety of input values were pregenerated for use as training data.

In the regression context, gradient boosting works by sequentially fitting a sequence of shallow binary trees to the errors committed by the current model formed by the weighted sum of previously fitted trees; to initialize the model, a single tree with zero splits—or equivalently a constant valued function—is provided as an initial guess. Each shallow (i.e., a depth of about 8 or less) tree can be seen as a coarse, piecewise constant function with each split representing a jump. Each tree is a relatively poor predictor of a complicated, nonlinear function and, as such, is referred to as a "weak learner." From a high level, each tree in the sequence corrects mistakes made by the previous trees. Each time a new tree is added to the ensemble, a 1D optimization problem is solved to determine the tree weight so that the overall model sum of squares error is minimized. In this way, as a larger number of trees are added to the boosted model, and the weighted sum over all trees converges to a better and better predictor of the training dataset. The inclusion of many small trees is why a gradient boosted model is sometimes called an ensemble-of-trees technique. Techniques that prevent data over-fitting are described in Friedman [13]. One such technique is to introduce a learning rate parameter, which reduces the contribution of each tree in the ensemble. A second strategy to prevent over fitting is "bagging," which is achieved by sampling the training data without replacement at each tree-fitting step so that each tree is fit to a randomly selected subset of the total training data available.

\subsubsection{Gradient Boosting Model Construction Results}

The gradient boosting implementation available in the scikit-learn Python package was used for this work [25].

The selected gradient boosted hyperparameters are summarized in Table (7). These are a potential avenue for future work to tune the model hyperparameters and improve the ability of the model to generalize to previously unseen data.

Table 7. Hyperparameters for the gradient boosting model.

\begin{tabular}{lc}
\hline Parameter name & Value or type \\
\hline Number of estimators & 2,000 \\
Maximum depth & 8 \\
Learning rate & 0.01 \\
Subsample & 0.7 \\
Loss function & Least squares \\
Criterion & Friedman MSE \\
\hline
\end{tabular}

A measure of relative variable importance is available by counting how many times all the constitutive trees split on a particular dimension. Each split is weighted by the improvement in RMS, and that particular split yielded to form a split-gain metric. The split-gains are tallied over all trees to provide an estimate of relative variable predictive power. In a gradient boosting model, this computation of relative variable importance has little additional computational cost since the number of splits and improvement in 
RMS per split can be logged as the model is fitted. All three datasets are trained by using the gradient boosting model with the hyperparameters in Table 7. Pre-processing the input variables to the surrogate model using polynomial combinations (default degree $=2$ ) of the features generally leads to a better fit of the training data. Therefore, polynomial features were used for pre-processing the input variables to the surrogate model for the Ris $\varnothing$ tests. A different number of datasets were used to train the three tests. An engineering approximation of 50 tests per multiplier was used to set the baseline for the number of training datasets. The Latin hypercube sampling method [6] was used to generate random samples for all the five multipliers, similar to the approach in Wu et al. [42]. The approximate number of training datasets required for the three tests are the following: 300 cases with 43 evaluations per set of multipliers for PSBT single-channel tests, 300 cases with 243 evaluations per set of multipliers for BFBT Assembly Type 1 tests, and 600 with 81 evaluations per set of multipliers for the Ris $\varnothing 200$ series tests. A comparison of the surrogate model fits for CTF test data is shown in Figures 26-28 for the three tests. For all three tests, the boundary conditions are the most sensitive parameters for the surrogate model. This is expected for two-phase tests and, in particular, wall boiling tests. The variable of importance generated for the Ris $\emptyset$ tests (Figure 29) was generated before turning on polynomial features, which are difficult to interpret given the increased number of independent variables. Although the surrogate model for the PSBT single-channel tests shows low sensitivity (less than a percent to a few percent) to all the five multipliers, the relative importance among the multipliers approximately match the sensitive analysis conducted via CTF for the bounding values, as summarized for PSBT single-channel tests in Table 4.

\subsection{Calibration Results}

The DREAM-based calibration was performed by using 10 chains executed for 20,000 generations, resulting in a total of 200,000 samples. The first 2,000 generations were discarded as burn-in generations to allow the chains to explore the parameter space and to converge to the stationary posterior density function. The parameter bounds for the five multipliers are given in Table 8. These values are chosen after performing an approximate sensitivity analysis via CTF for the three tests chosen for calibration. Furthermore, larger optimum multipliers indicate considerable model deficiency, and thus the chosen bounds can be considered an acceptable engineering approximation.

Table 8. Parameter bounds for calibration

\begin{tabular}{lcc}
\hline Multiplier name & Lower bound & Upper bound \\
\hline k_xkwlxA (theta_1) & 0.1 & 2.0 \\
k_xkwlxB (theta_2) & -3.0 & -1.0 \\
k_xk_sb (theta_3) & 0.1 & 2.0 \\
k_xk_slg (theta_4) & 0.1 & 2.0 \\
k_xk_anrflm (theta_5) & 0.1 & 2.0 \\
\hline
\end{tabular}

An approximate convergence criteria was used, which involved comparing the distributions for different sample sizes (e.g., 1.5e5 and 2e5 samples). The calibration was considered to be finished when it was observed that the distributions and the median values did not change significantly sample to sample. The calibration results are shown in Figures 30 and 31. Figure 30 depicts each of the 10 chain positions as a function of DREAM sample generation. As mentioned previously, 20,000 generations are shown 


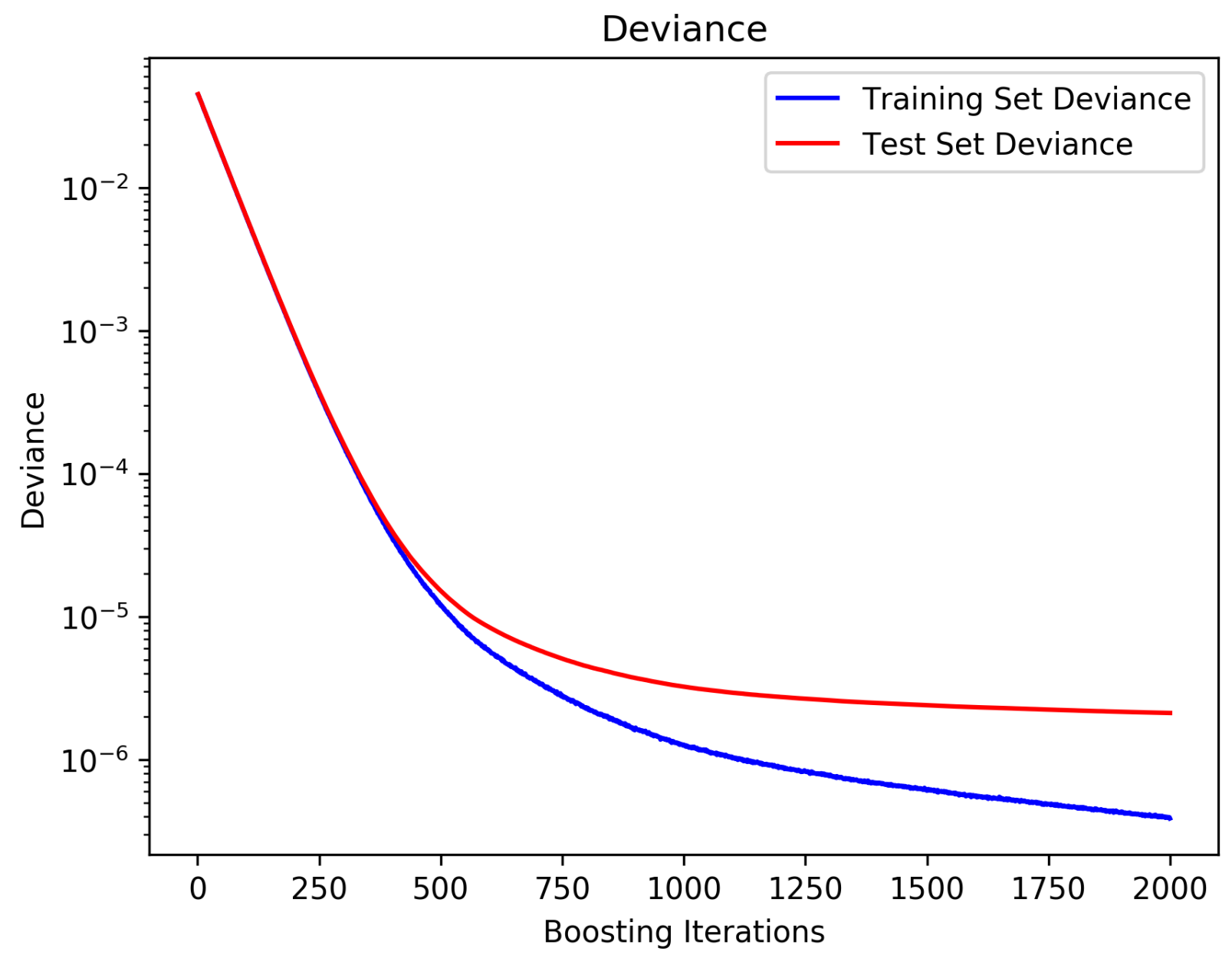

Figure 26. Change in the training and testing RMSE as a function of the number of trees for the gradient boosted surrogate model for the PSBT single-channel tests. 


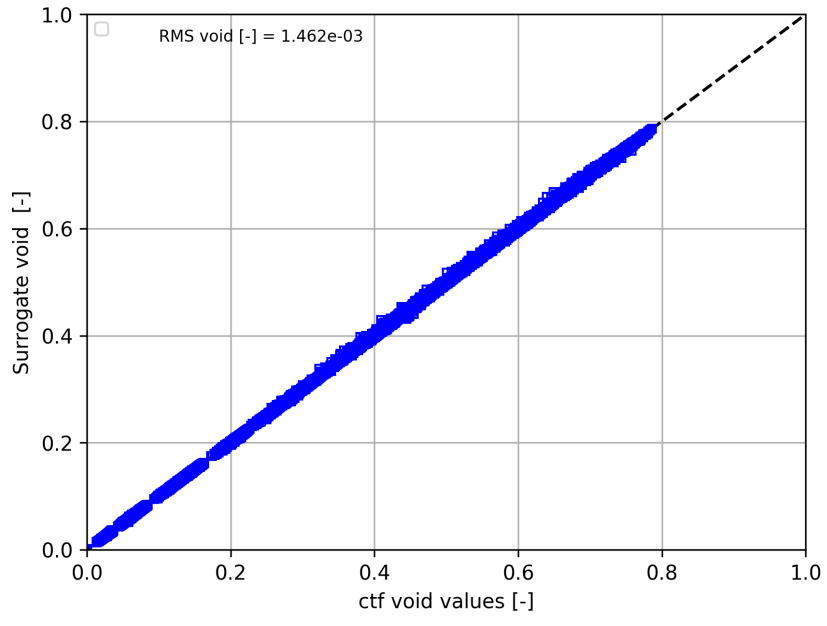

(a) Surrogate fit vs. CTF void fraction (test data).

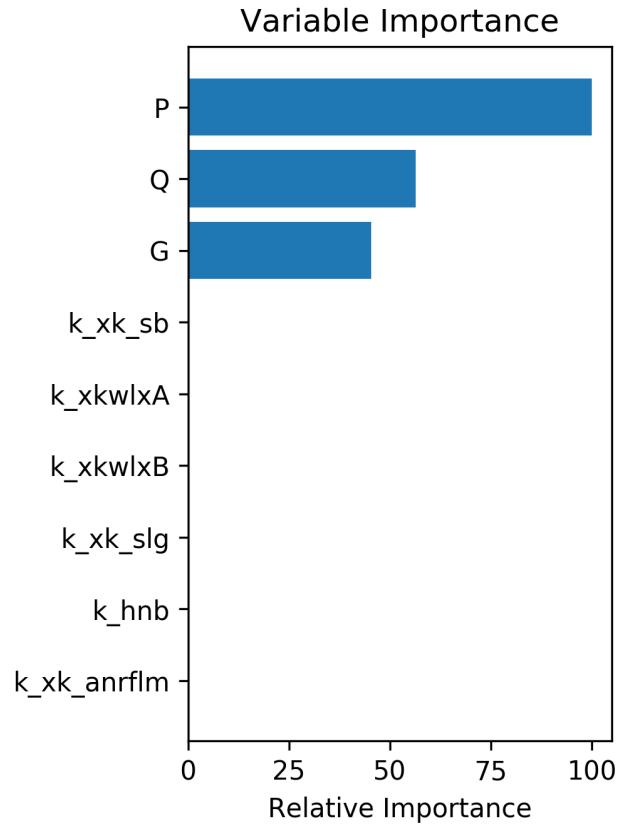

(b) Variables of importance for the surrogate model.

Figure 27. Surrogate model for PSBT single-subchannel tests (void fraction).

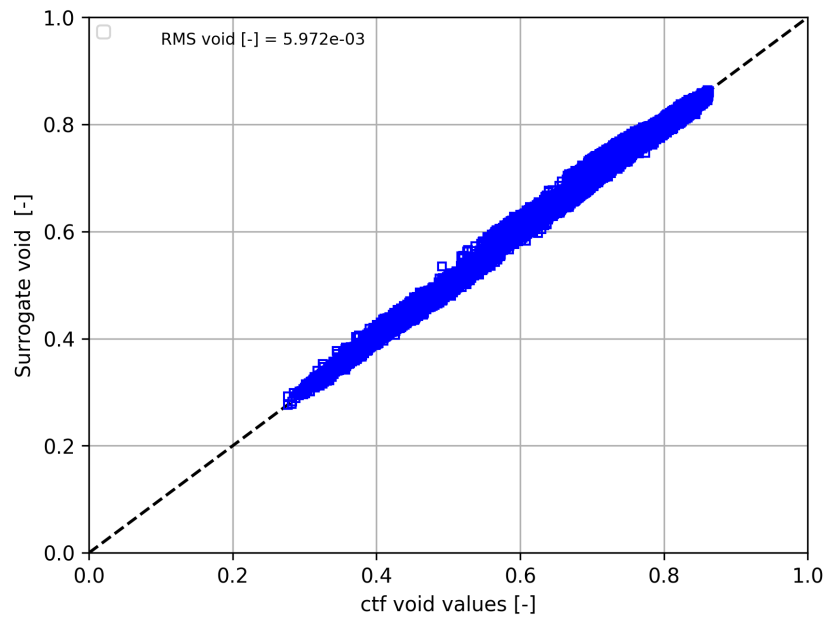

(a) Surrogate fit vs. CTF BFBT subchannel void fraction data (test data).

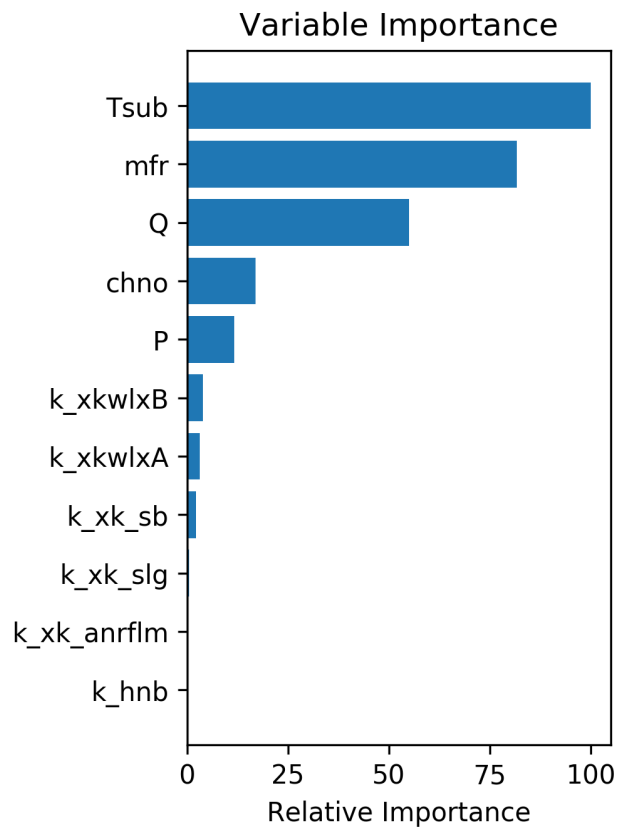

(b) Variables of importance for the surrogate model.

Figure 28. Surrogate model for BFBT void distribution tests (void fraction). 


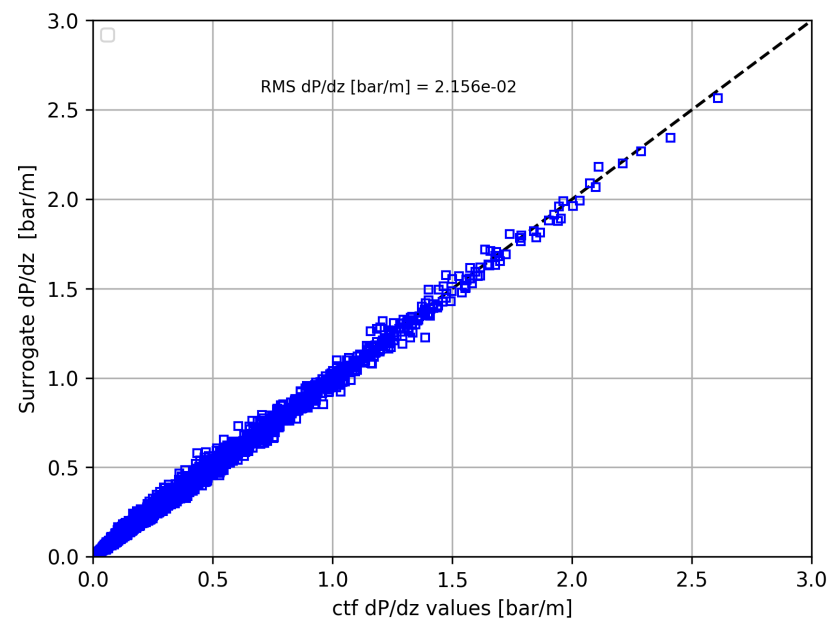

(a) Surrogate fit vs. CTF pressure gradient (test data).

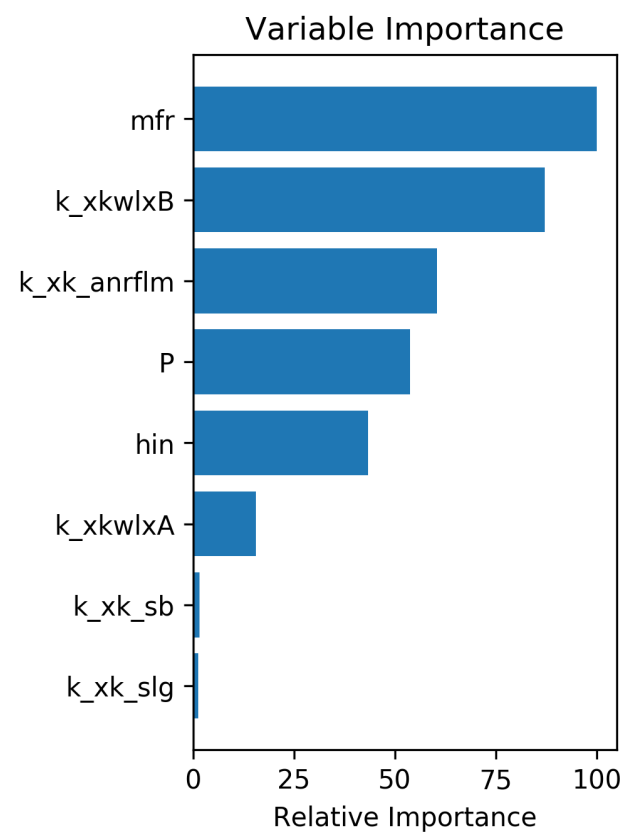

(b) Variables of importance for the surrogate model.

Figure 29. Surrogate model for Risø 200 series (adiabatic) tests (two-phase pressure gradient).

(horizontal axis). A dashed vertical line in Figure 30 indicates the burn-in generation; samples before this line were discarded in the generation of the posterior distribution shown in Figure 31. In the pair-wise posterior distribution plot in Figure 31, the 0.05, 0.5, and 0.95 quantiles for each marginal distribution are given. The 0.5 quantile $\left(q_{50}\right)$ is shown as a vertical dotted line in each marginal distribution plot, and the mean value $(\mu)$ is shown as a vertical dashed line. These are preliminary results, and several observations can be made regarding the results. First, for each of the five parameters, the chains do not mix well with the increasing number of samples and stay at a constant value, indicating that the sample acceptance rate is very low. This can be seen in the marginal posterior distributions in which all the multipliers have sharp and possibly multimodal posterior distributions. Second, the pair-wise distributions are not smooth and have clustered samples. This could be due to the gradient boosting method, which is used as the surrogate model in this work. Gradient boosting models produce a piecewise constant prediction surface, which results in the likelihood function defined in Eq. (40) to be non-smooth, and consequently, the posterior distribution given by Eq. 43 is non-smooth in this case. This poses a significant challenge to the MCMC sampler since discontinuous pockets of high likelihood inhibit the mixing of the chains because proposed samples must land precisely in an area of high probability to have any reasonable chance of acceptance. Alternative machine learning tools, such as support vector machines and Guassian process regression (both available in scikit-learn), could result in smoother posterior distributions. Applying these surrogate construction techniques is an avenue for future work.

The median values - hereafter referred to as optimum values - along with the q5 and q95 quantiles and standard deviation for the five multipliers are listed in Table 9. There are three findings regarding the optimum values. First, all the five optimum values lie well within the chosen parameter space, justifying 
the bounds used for calibration. Second, the optimum values for the three interfacial drag multipliers as currently defined indicate that interfacial drag is considerably overpredicted in the small bubble and slug flow regime and, to a lesser extent, in the annular flow regime. This is of consequence in low void fraction regimes, as typified by the PSBT single-channel tests. This is consistent with the findings in previous sensitivity studies and with the assumption that interfacial drag is being overpredicted by the legacy CTF models. The annular-mist interfacial drag overprediction is significantly reduced due to the fix discussed in Section 5. The optimum values must be treated with caution since they are a consequence of the chosen tests, multipliers, and sensitivity and accuracy of the surrogate models. Last, the optimum wall friction drag multiplier indicates an overprediction in the two-phase pressure-drop prediction with the default CTF model. As observed in Section 5, even after fixing the issue with the unstable film interfacial drag, a slight overprediction in the two-phase pressure drop still existed.

Table 9. Summary of calibration quantile and standard deviation values. Optimal values taken as the median (q50) values.

\begin{tabular}{lcccc}
\hline Multiplier name & q5 value & q50 value & q95 value & $+/-1 \sigma$ \\
\hline k_xkwlxA (theta_1) & 0.2428 & 0.3234 & 0.9529 & 0.2538 \\
k_xkwlxB (theta_2) & -2.482 & -2.2412 & -1.645 & 0.2742 \\
k_xk_sb (theta_3) & 0.1174 & 0.2668 & 0.9809 & 0.2782 \\
k_xk_slg (theta_4) & 0.1064 & 0.3810 & 0.9647 & 0.3306 \\
k_xk_anrflm (theta_5) & 0.6147 & 0.6810 & 1.1531 & 0.1602 \\
\hline
\end{tabular}

The complete validation database in CTF was run via the optimum values to gauge their impact on the CTF predictions of experimental data. The results with the base model with multipliers set to one and with the multipliers set to values from Table 9 are shown in the following eight figures for select tests from the validation database.

Generally, the calibrated model results in improved void fraction predictions, especially for the tests used for calibrating the multipliers. This is evident from the improved void fraction predictions for the PSBT single-channel tests in Figure 32, the BFBT void distribution tests (subchannel void fractions) in Figure 36, and the BFBT bundle average void fraction predictions for the void distribution tests in Figure 37.

Although the BFBT bundle tests were included in the calibration, only Assembly Type 1 results were used for calibration. The validation results, which tested the optimized parameters for all assembly types (Figure 37), show that a significant improvement in RMSE is achieved for every assembly type, which includes different power shapes and geometries from the type used in calibration.

The impact of the multipliers for tests that are not part of the calibration process is shown for the PSBT bundle tests series 5 in Figure 33, series 6 in Figure 34, and series 7 in Figure 35. There is good improvement in the bundle-average void fraction measurements at the lowest axial location (small bubble and slug flow), and the impact is lowest for the uppermost axial location at which the flow regime is churn turbulent. There is modest improvement in the mean void fraction error prediction for all three series. There is negligible change in the void mean error for the Bartolomei tests, which are shown in Figure 38. Because the base model underpredicts the void fraction at lower void fractions, the calibrated model is expected to worsen the underprediction in this flow regime. The void fraction predictions are considerably improved in the higher void fraction regions (i.e., greater than 0.3 ). The improved higher void predictions are likely due to the improved interfacial drag values. The lower void regions tend to be more affected by 

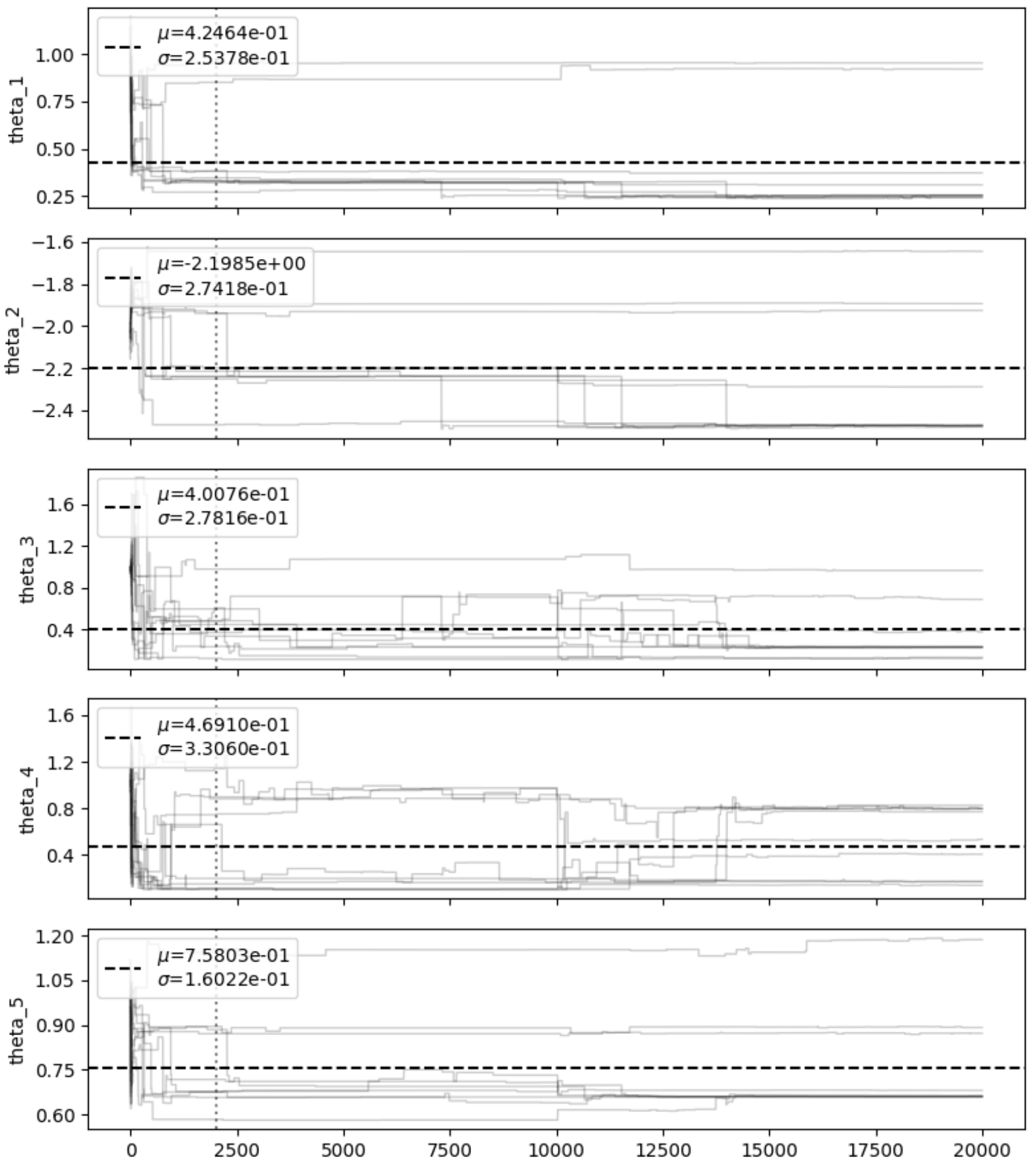

Figure 30. Change in the distribution of calibration multipliers for different chains as a function of sample size per chain. 


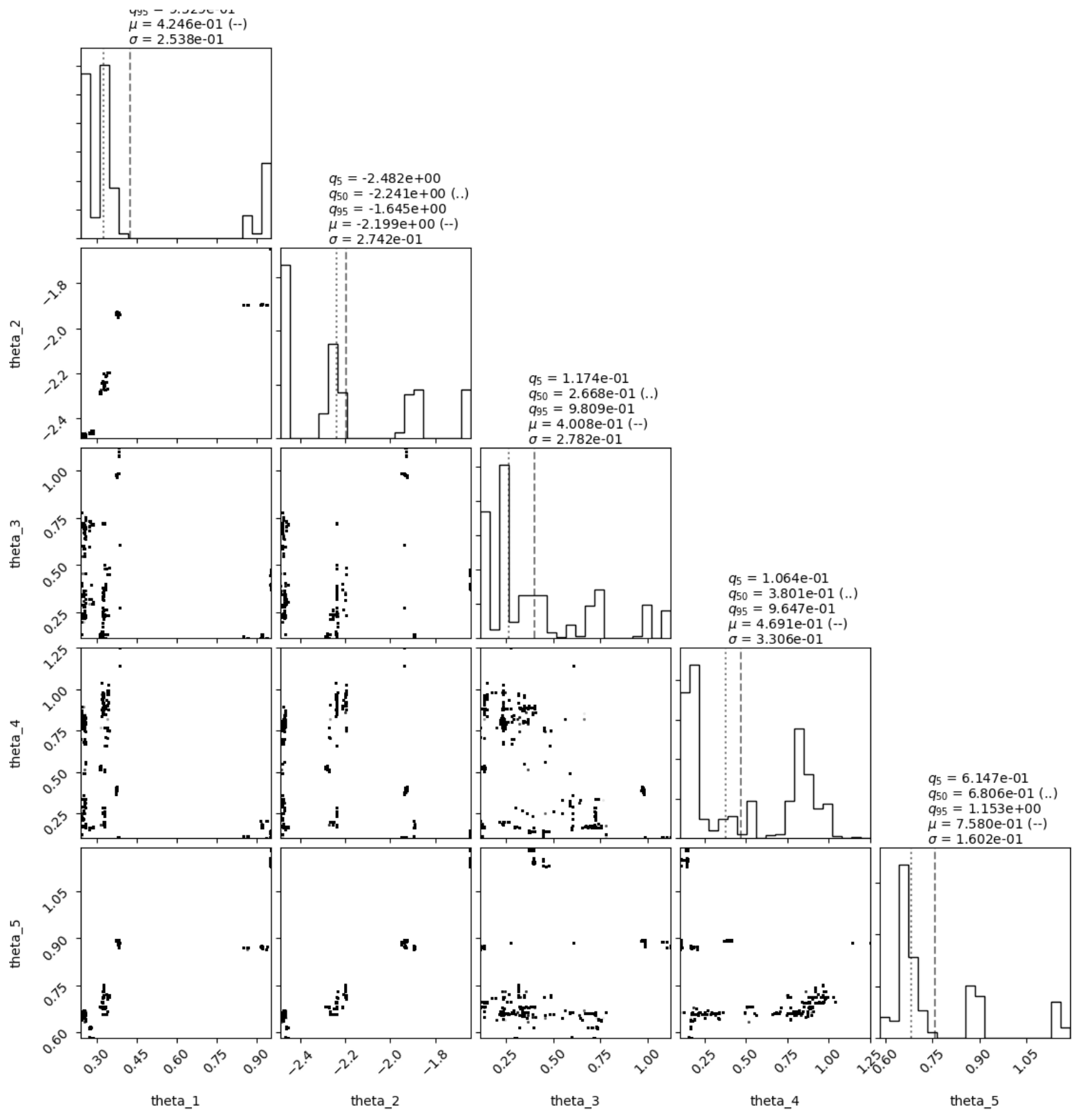

Figure 31. Final posterior distributions (pair-wise and marginal) of the calibration multipliers. 


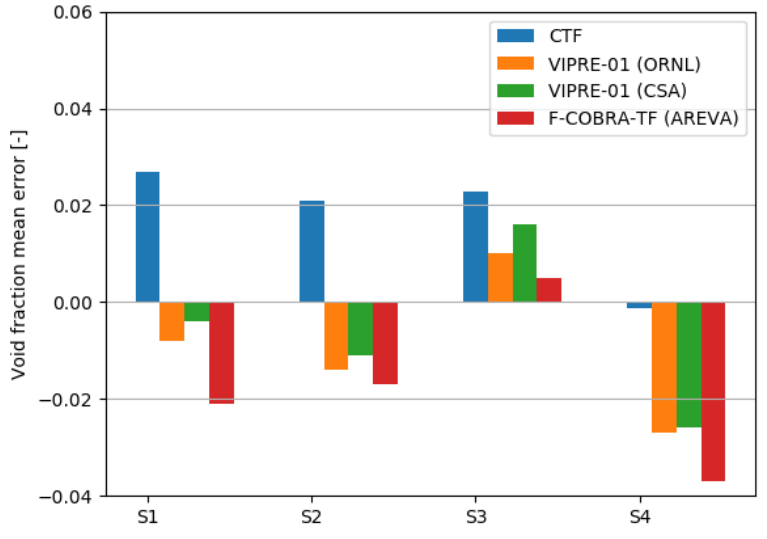

(a) Base model.

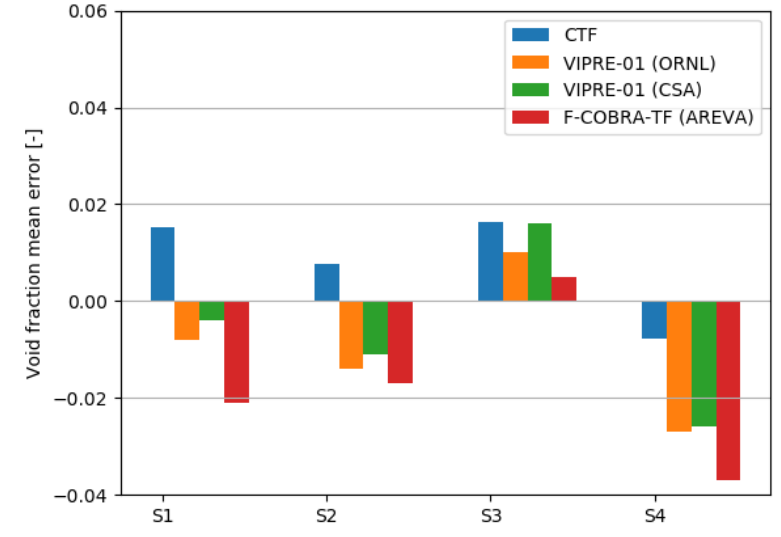

(b) Calibrated model.

Figure 32. Comparison of PSBT subchannel void fraction prediction mean error for different codes.

the subcooled boiling model, which was not included in this study. The multiplier on the model did not largely impact the results in the included tests. The Bartolomei tests might have a sensitivity to this multiplier, or the multiplier might not be affecting the vapor generation component of the model. The boiling model will be studied further in future calibration efforts. Overall, all of the void prediction tests indicate that interfacial drag models with more targeted tunable model coefficients, such as the drift flux model, could result in improved predictions and make the model extensible to different operating conditions and geometries. For example, the flow regime-dependent $k$-coefficient in the drift-flux model in Eq. (34) could be directly tuned, which is similar to what was done for the two-phase multiplier parameters.

There is an excellent four-fold improvement in the two-phase pressure-drop prediction for the Ris $\emptyset$ tests. This is driven by the low k_xkwlxA optimum value and better void fraction predictions in the annular flow regime via the liquid film interfacial drag multiplier. Finally, the wall temperature predictions are shown for the Rohsenow tests (Figure 40), WALT tests (Figure 41), and the Thom tests (Figure 42). For all three tests, the calibrated model predictions are largely unchanged from the base model predictions. The wall temperature predictions are driven by the wall heat transfer model, which is unchanged from the base model, resulting in similar predictions. Further improvements to the wall heat transfer model are required to improve the wall temperature predictions, which could be a part of a future calibration study. 


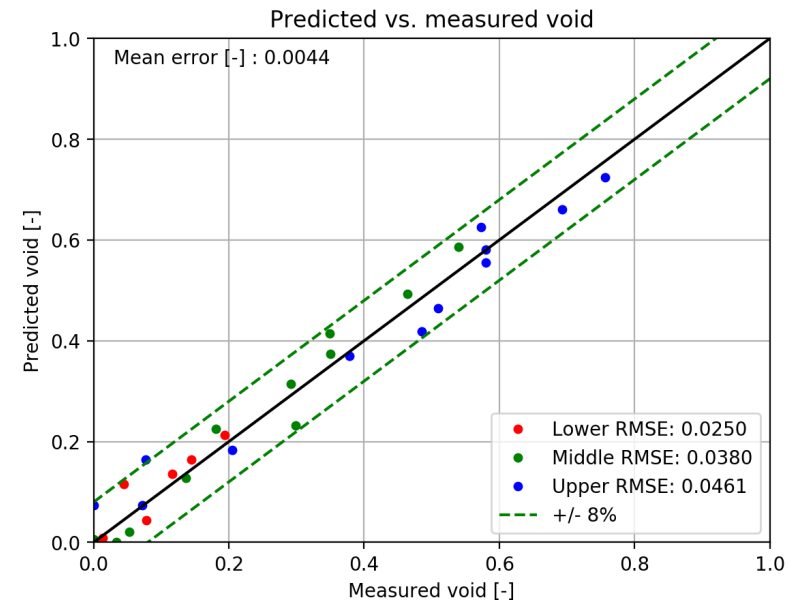

(a) Base model.

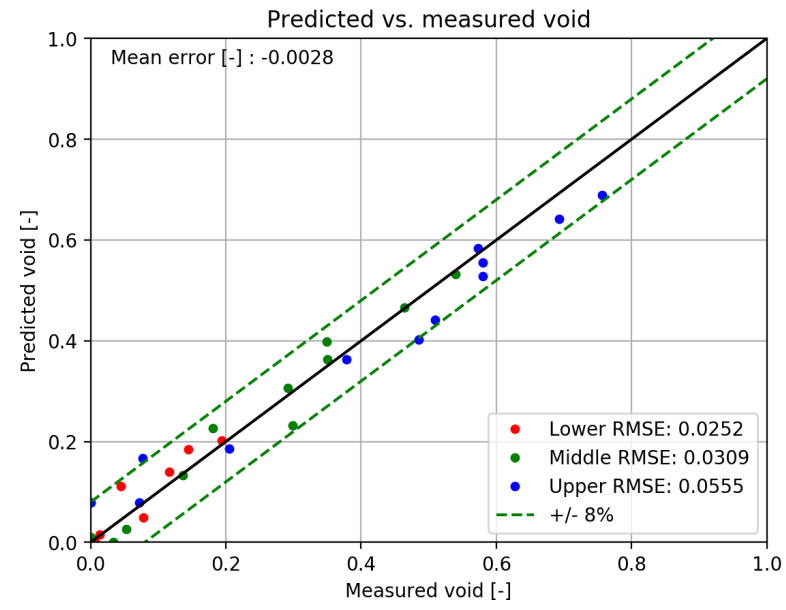

(b) Calibrated model.

Figure 33. Comparison of PSBT series 5 bundle void fraction prediction with the calibrated model.

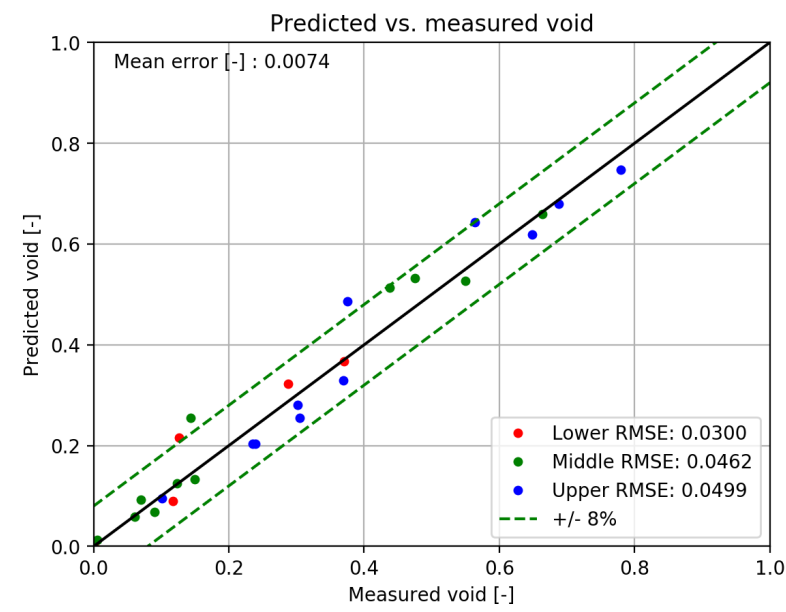

(a) Base model.

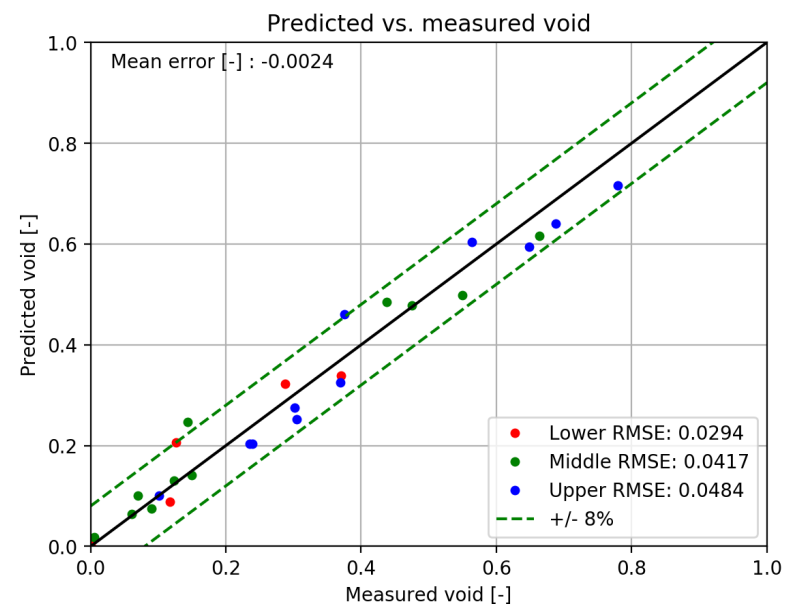

(b) Calibrated model.

Figure 34. Comparison of PSBT series 6 bundle void fraction prediction with the calibrated model. 


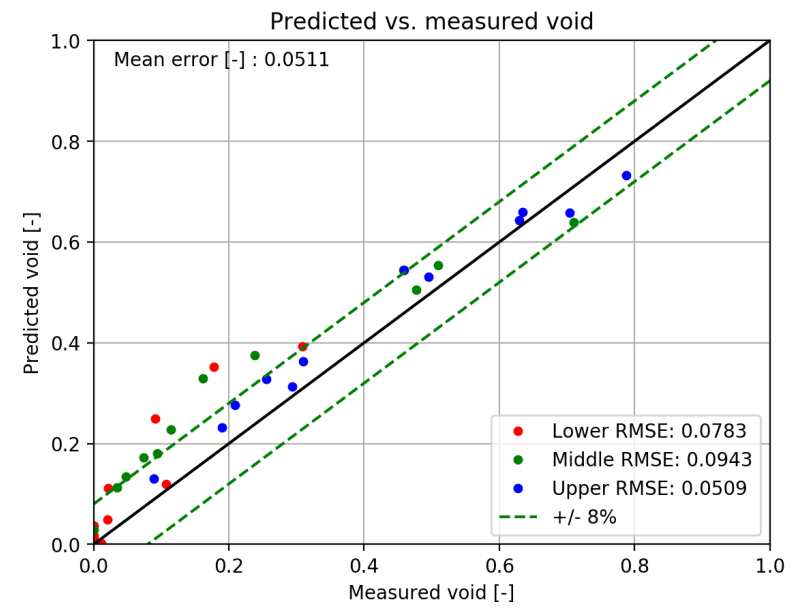

(a) Base model.

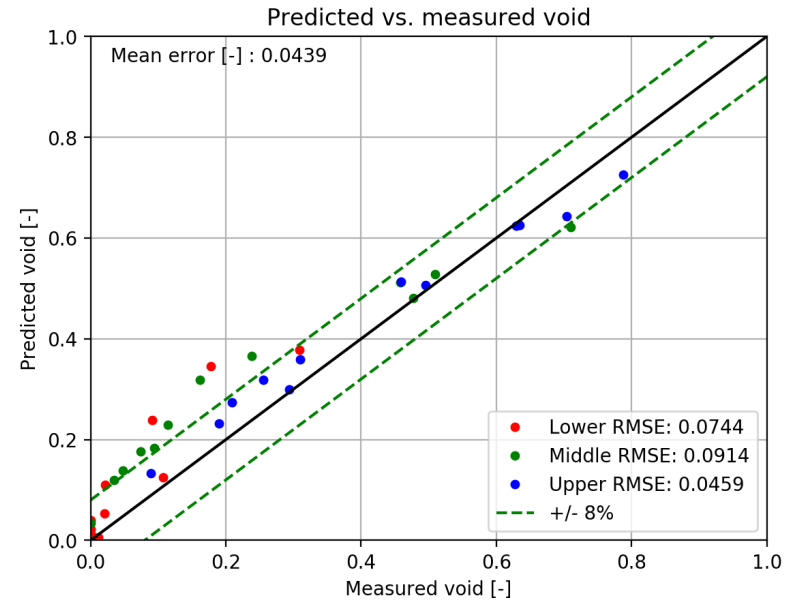

(b) Calibrated model.

Figure 35. Comparison of PSBT series 7 bundle void fraction prediction with the calibrated model.

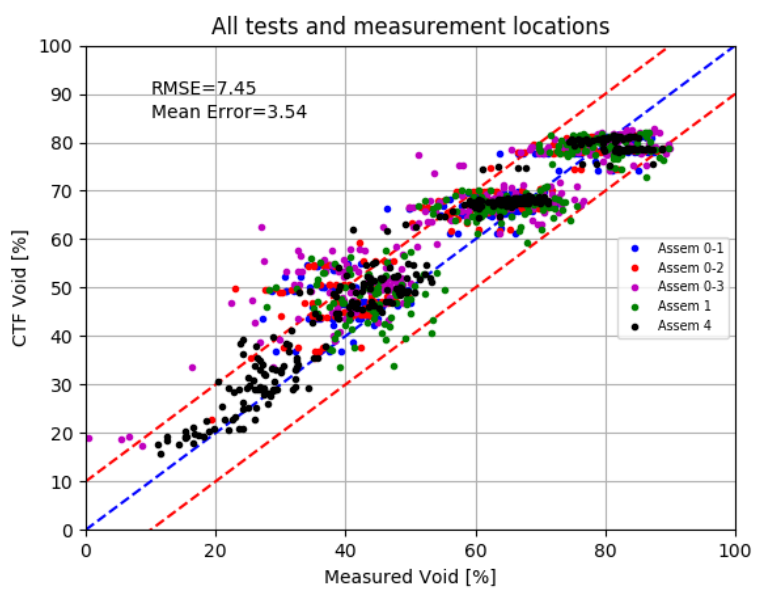

(a) Base model.

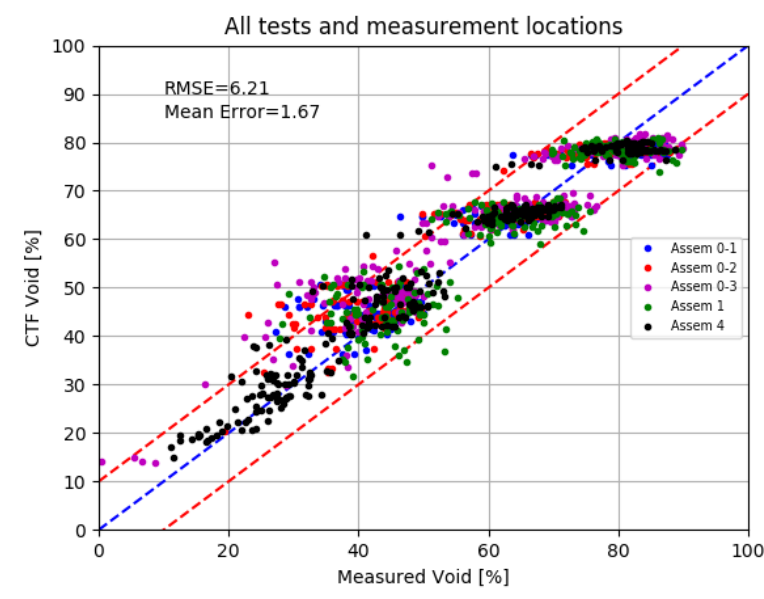

(b) Calibrated model.

Figure 36. Comparison of BFBT subchannel void fraction prediction with the calibrated model. 


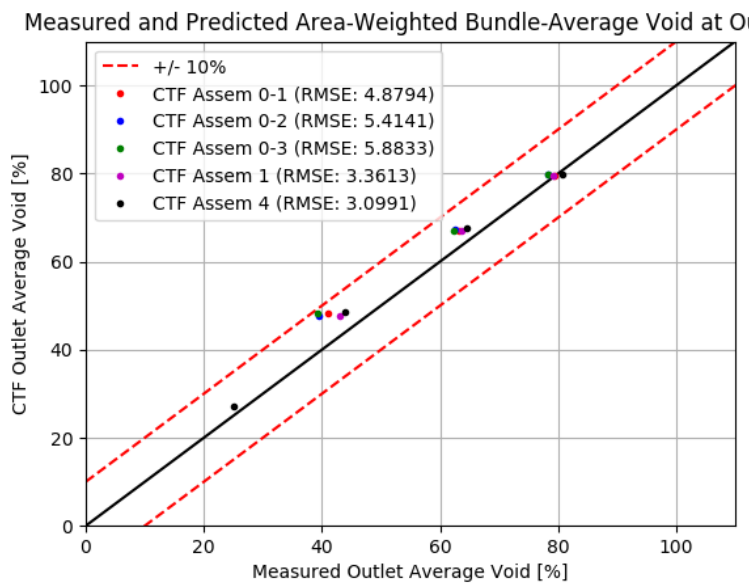

(a) Base model.

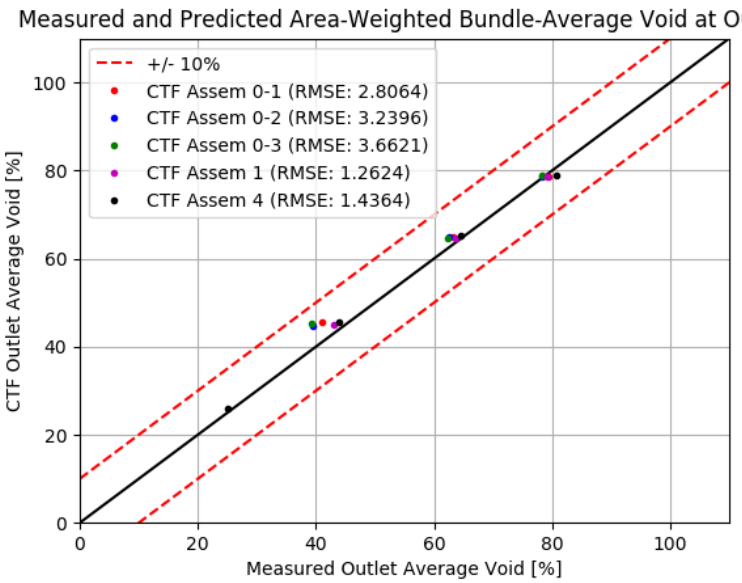

(b) Calibrated model.

Figure 37. Comparison of BFBT bundle average outlet void fraction prediction with the calibrated model.

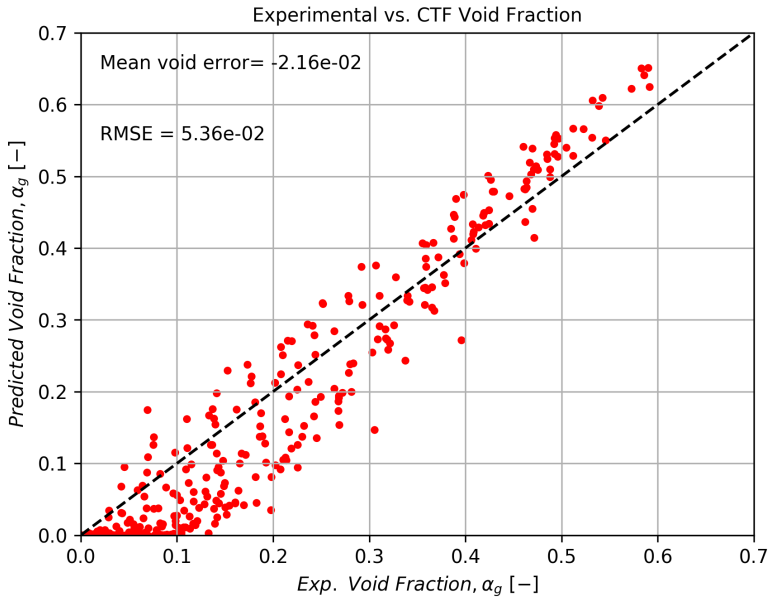

(a) Base model.

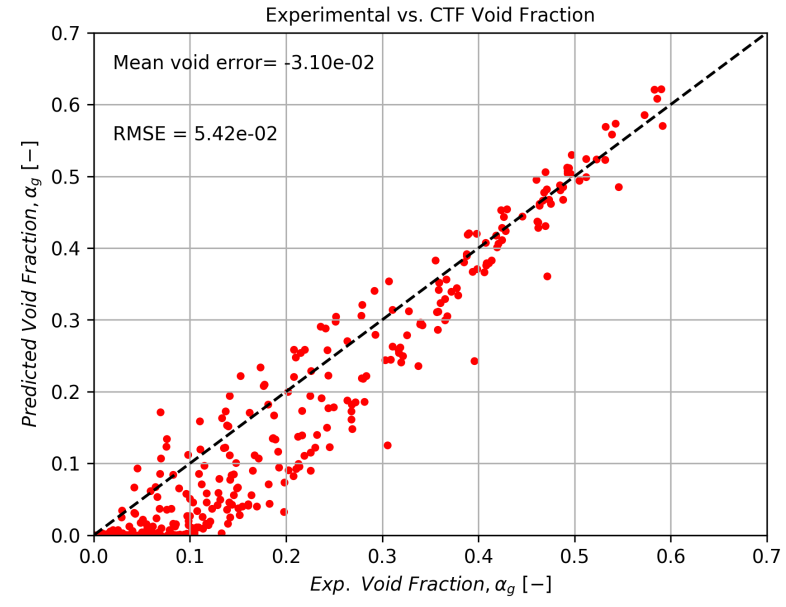

(b) Calibrated model.

Figure 38. Comparison of Bartolomei void fraction prediction with the calibrated model. 


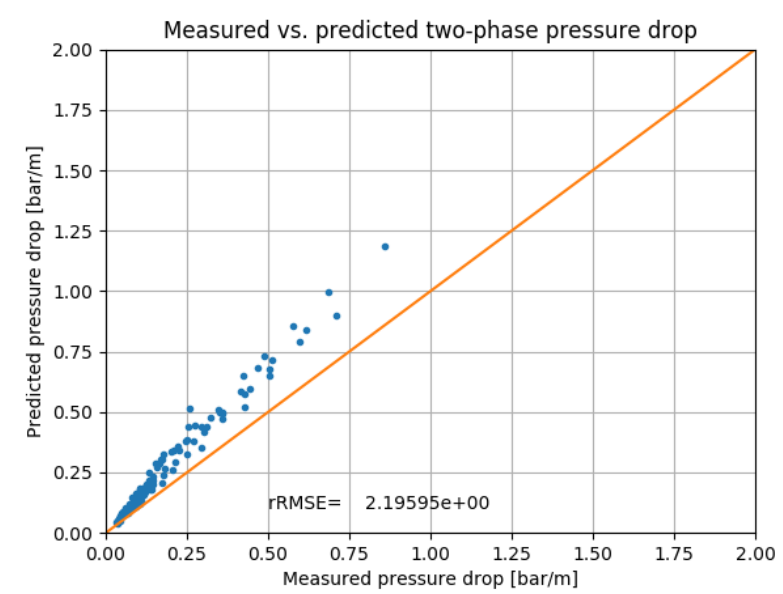

(a) Base model.

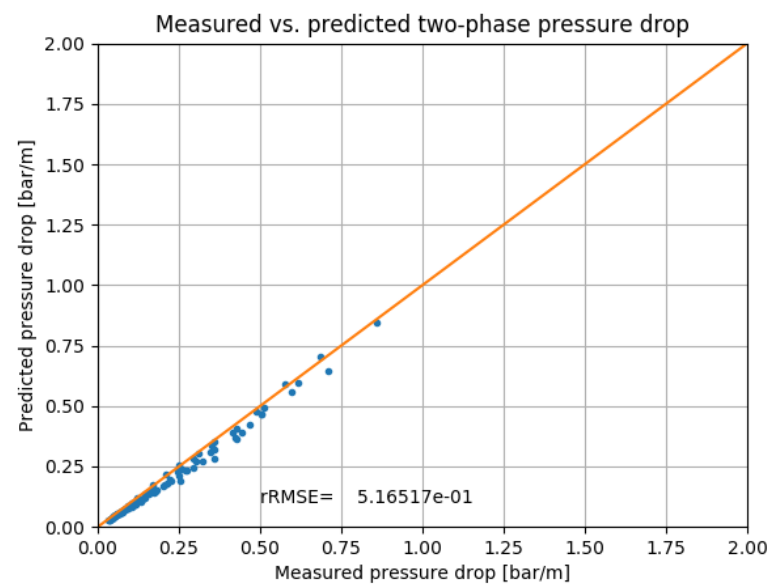

(b) Calibrated model.

Figure 39. Comparison of Risø 200 series two-phase pressure-drop prediction with the calibrated model.

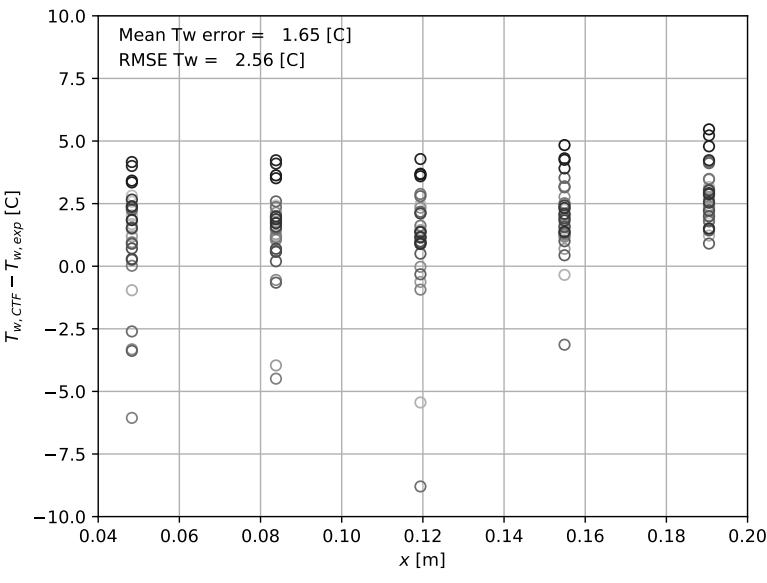

(a) Base model.

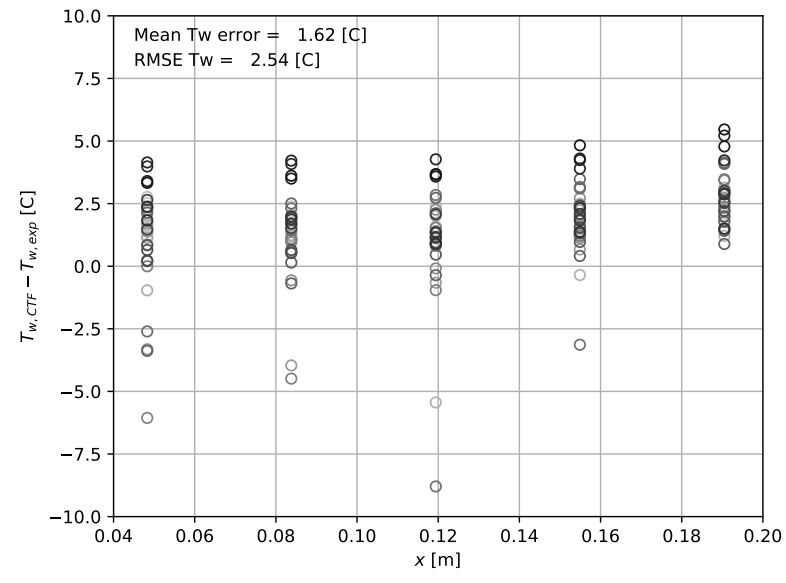

(b) Calibrated model.

Figure 40. Comparison of Rohsenow tests wall temperature prediction with the calibrated model. 


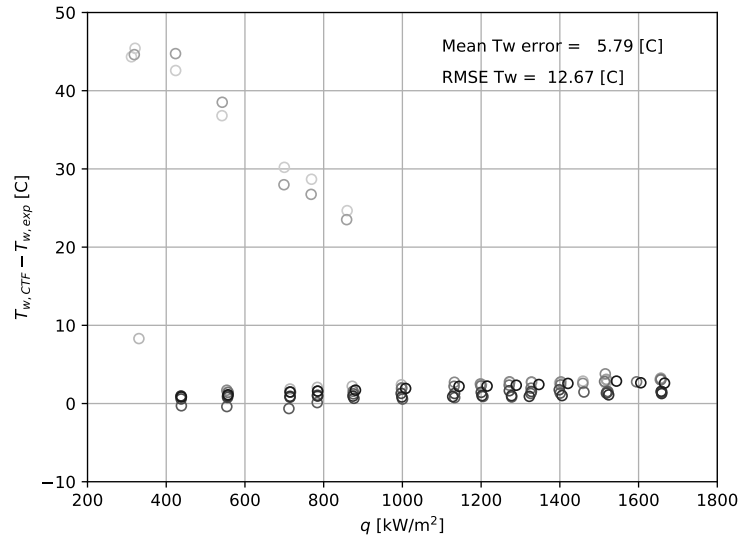

(a) Base model.

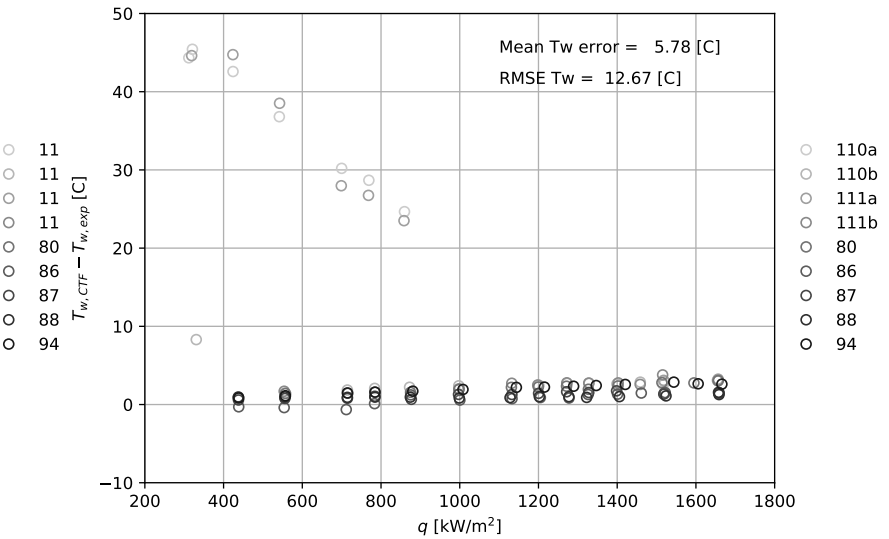

(b) Calibrated model.

Figure 41. Comparison of WALT tests wall temperature prediction with the calibrated model.

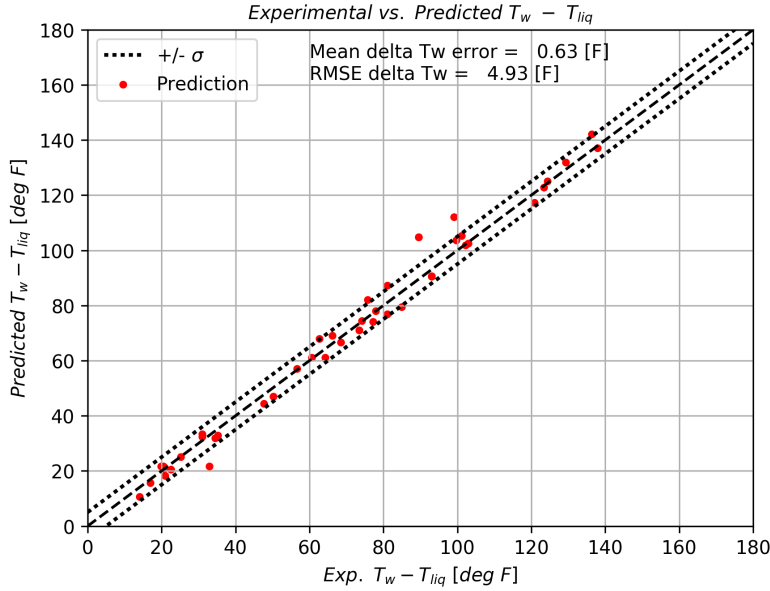

(a) Base model.

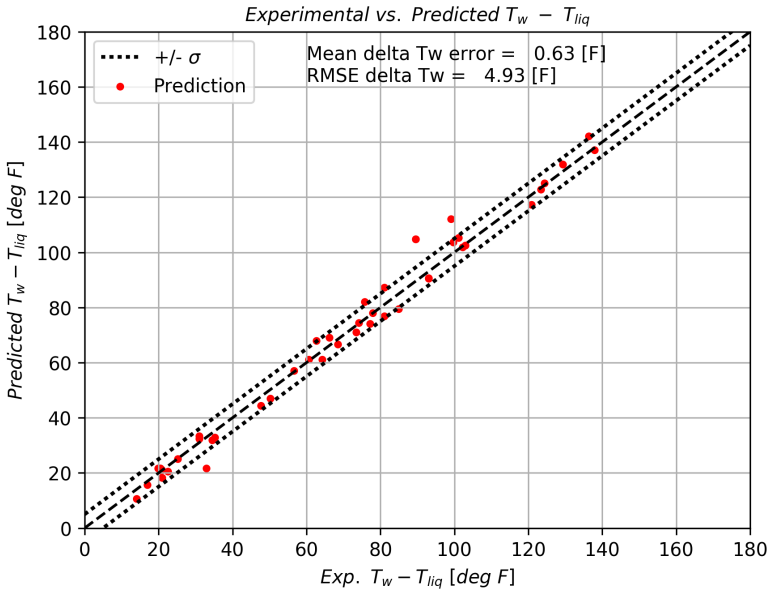

(b) Calibrated model.

Figure 42. Comparison of Thom tests delta wall temperature prediction with the calibrated model. 



\section{SUMMARY}

This milestone's goal was to improve CTF void prediction in BWR conditions by improving relevant two-phase closure models. Previous validation efforts indicated that CTF tends to overpredict the void in bubbly flow regimes and pressure drop in annular flow. Previous studies also indicated that the subcooled boiling model and the interfacial drag models significantly impact predicted results. Therefore, efforts focused on improving these models. Two paths were taken to improve the models.

The first path involved searching the literature for more physically correct and accurate closure models to add as options to the legacy models already in CTF. Based on this work, the Basu ONB model with the Gorenflo boiling heat transfer model and Saha-Zuber bubble detachment criteria were chosen to implement as an alternative subcooled boiling model. Additionally, a drift-flux model was used to derive a new set of interfacial drag coefficients that would be used to define the slip between vapor and liquid phases. An extensive assessment of the effect of these modeling changes was performed and has shown a significant improvement to void and wall temperature prediction.

Moving forward, the testing of the new ONB and drift-flux models will be expanded to large-scale BWR models that are modeled via CTF in a coupled sense as part of VERA. Testing will also be expanded to additional CTF validation tests that were not of primary interest during this work to ensure that the models are robust and widely applicable. After successful testing, the default models for interfacial drag and wall heat transfer prediction in VERA will be transitioned to using these improved models.

In addition to these two models, some work was briefly done to review the annular-mist flow regime interfacial drag and churn-to-annular flow regime transition criteria. An issue was identified and fixed with the treatment of unstable film interfacial drag, which led to a marked improvement of Risø two-phase pressure-drop prediction. This also identified issues with the determination of the flow regime transition void, which is currently allowed to occur at too high a void fraction. Additionally, the churn-turbulent interpolation region is likely too large as currently posed. These finding identify additional future work that will be needed to improve annular flow prediction, which is critical for successful BWR analysis.

The second path for improving closure models used a Bayesian calibration process to optimize selected modeling parameters to achieve an improved prediction of existing two-phase experimental data available in the CTF validation matrix. Selected parameters included multipliers on interfacial drag models in three different flow regimes for void improvement and parameters in the two-phase multiplier model for two-phase pressure-drop improvement. Calibration experiments included PSBT single-channel tests, one assembly type from the BFBT bundle void distribution tests, and the unheated Ris $\emptyset$ boiling experiments. Surrogates were developed to use in place of CTF for each facility when performing the calibration. The calibration yielded a set of optimized parameters, which were then used to rerun the validation tests. Generally, void prediction was improved for tests that were both included and not included in the calibration datasets. Two-phase pressure-drop predictions in the Risø tests were also significantly improved.

The calibration work successfully set up a framework for calibrating two-phase models in CTF and improved prediction results. This work also identified several areas in which improvements can be made in future activities. First, the two-phase prediction results experienced the highest degree of improvements after calibration, which is likely because the modeling parameters were calibrated rather than a general multiplier. This also means that the model improvements are more likely to be extensible to other 
geometries and operating conditions. A similar approach for exposing tuneable modeling coefficients for the interfacial drag models and subcooled boiling model should be done in future work. Second, because the drift-flux model was not ready in time for the calibration study, the legacy interfacial drag models were used instead. Future work should involve switching to calibrating modeling parameters in the drift-flux model. Third, the posterior distribution of the calibration revealed that the surrogates tend to produce a non-smooth response, which results in clustered and sometimes bimodal responses. This is likely due to the gradient boosting technique that was used to train the surrogates in this study. Other methods are available and should be reviewed in future work to produce a better response and improved calibration. 


\section{REFERENCES}

\section{References}

[1] S. Bajorek et al. TRACE V5.1051 Theory Manual. US NRC, 2016.

[2] G.G. Bartolomei, V.G. Brantov, Y.S. Molochnikov, Y.V. Kharitonov, V.A. Solodkii, G.N. Batashova, and V.N. Mikhailov. An Experimental Investigation of True Volumetric Vapour Content with Subcooled Boiling in Tubes. Thermal Engineering, 29:20-22, 1982.

[3] N. Basu, G.R. Warrier, and V.K. Dhir. Onset of Nucleate Boiling and Active Nucleation Site Density During Subcooled Flow Boiling. J Heat Transfer, 124:717-728, 2002.

[4] A. Bergeron, T. Chataing, E. Dècossin, J. Garnier, P. Pèturaud, and S. K. Yagnik. Design, feasibility, and testing of instrumented rod bundles to improve heat transfer knowledge in pwr fuel assemblies. In Proceedings of the 2007 International LWR Fuel Performance Meeting, San Francisco, California, USA, September 30-October 3, 2007, 2007.

[5] R.W. Bjorge, G.R. Hall, and W.M. Rohsenow. Correlation of forced convection boiling heat transfer data. Int J Heat and Mass Transfer, 25:753-757, 1982.

[6] Mohamed Amine Bouhlel, John T. Hwang, Nathalie Bartoli, RÃl'mi Lafage, Joseph Morlier, and Joaquim R. R. A. Martins. A python surrogate modeling framework with derivatives. Advances in Engineering Software, page 102662, 2019. ISSN 0965-9978. doi: https://doi.org/10.1016/j.advengsoft.2019.03.005.

[7] Benjamin S. Collins, William Gurecky, Lindsay N. Gilkey, Alicia Elliott, and Dave J. Kropaczek. Inference of crud model parameters from plant data. Proceedings from ANS Global/Top Fuel Conference. Seattle, WA., Sep 2019.

[8] US Nuclear Regulatory Commission. Trace v5.840 theory manual: Field equations, solution methods, and physical models. Technical report, US Nuclear Regulatory Commission, 2013.

[9] J. Deshon. Simulated fuel crud thermal conductivity measurements under pressurized water reactor conditions. Technical Report 1022896, EPRI, 2011.

[10] GE Hitachi Nuclear Energy. Tracg model description. Technical Report NEDO 32176 rev 4, GE Hitachi Nuclear Energy, 2008.

[11] Daniel Foreman-Mackey, David W. Hogg, Dustin Lang, and Jonathan Goodman. emcee: The MCMC Hammer. Publications of the Astronomical Society of the Pacific, 125(925):306, March 2013. doi: 10.1086/670067.

[12] J.H. Friedman. Greedy function approximation: A gradient boosting machine. Annals of Statistics, 29:1189, October 2001.

[13] J.H. Friedman. Stochastic gradient boosting. Computational Statistics and Data Analysis, 38:367, Febuary 2002.

[14] G.G.Bartolomei and V.M. Chanturiya. Experimental Study of True Void Fraction When Boiling Subcooled Water in Vertical Tubes. Thermal Engineering, 14:123-128, 1967. 
[15] D. Gorenflo and D. Kenning. VDI Heat Atlas. VD-Verlag GmbH, Springer, Dusseldorf, 2nd edition, 1993.

[16] W.H. Henstock and T.J. Hanratty. The interfacial drag and the height of the wall layer in annular flows. AIChE Journal, 22(6):990 - 1000, 1976.

[17] M. Ishii. One-dimensional drift-flux model and constitutive equations for relative motion between phases in various two-phase flow regimes. Technical Report ANL-77-47, Argonne National Laboratory, September 1977.

[18] Isoa Kataoka and Mamoru Ishii. Drift flux model for large diameter pipe and new correlation for pool void fraction. Inf. J. Hear Mass Transfer. Vol. 30, No. 9, pp. 1927-1939, 1987.

[19] J. E. Kelly, S. P. Kao, and M. S. Kazimi. THERMIT-2: A Two-Fluid Model for Light Water Reactor Subchannel Transient Analysis. Technical Report MIT-EL-81-014, MIT Energy Laboratory Electric Utility Program, 1981.

[20] V. Kumar and R. Salko. Implementation of a new wall boiling model in ctf. In Transactions of American Nuclear Society, Chicago, IL, Nov 15-19, 2020, 2020.

[21] R.T. Lahey. A Mechanistic Subcooled Boiling Model. In Proc. Sixth Int. Heat Transfer Conference, volume 1, pages 293-297, 1978.

[22] J. Lane. The Development of a Comprehensive Annular Flow Modeling Package for Two-Phase Three-Field Transient Safety Analysis Codes. PhD thesis, The Pennsylvania State University, 2009.

[23] B. Neykov, F. Aydogan, L. Hochreiter, K. Ivanov, H. Utsuno, and F. Kasahara. NUPEC BWR Full-size Fine-mesh Bundle Test (BFBT) Benchmark. Technical report, NUCLEAR ENERGY AGENCY, 2006.

[24] OECD/NEA/NCS. International benchmark on pressurized water reactor sub-channel and bundle tests. volume ii: Benchmark results of phase iâǍŤvoid distribution. Technical report, Organization for Economic Co-operation and Development/Nuclear Energy Agency/Nuclear Science Committee, 2016.

[25] F. Pedregosa, G. Varoquaux, A. Gramfort, V. Michel, B. Thirion, O. Grisel, M. Blondel, P. Prettenhofer, R. Weiss, V. Dubourg, J. Vanderplas, A. Passos, D. Cournapeau, M. Brucher, M. Perrot, and E. Duchesnay. Scikit-learn: Machine learning in Python. Journal of Machine Learning Research, 12:2825-2830, 2011.

[26] N. Porter and L. Gilkey. Separate effects validation for subcooled boiling in ctf. Technical Report CASL-U-2020-1946-000, Consortium for Advanced Simulation of Light Water Reactors, 2020.

[27] Nathan W. Porter, Maria N. Avramova, and Vincent A. Mousseau. Uncertainty quantification study of $\mathrm{ctf}$ for the oecd/nea lwr uncertainty analysis in modeling benchmark. Nuclear Science and Engineering, 190(3):271-286, 2018. doi: 10.1080/00295639.2018.1435135. URL https://doi.org/10.1080/00295639.2018.1435135.

[28] D. W. Radcliffe R. T. Lahey Jr., B. S. Shirlakar. Two-Phase Flow and Heat Transfer In Multirod Geometries: Subchannel and Pressure Drop Measurements in a Nine-Rod Bundle for Diabatic and Adiabatic Conditions. Technical report, General Electric, 1970. 
[29] W. M. Rohsenow and J. A. Clark. Heat transfer and pressure drop data for high heat flux densities to water at high subcritical pressures. Technical Report N5ori-07827/NR035-267/DIC6627, Office of Naval Research, 1951.

[30] A. Rubin, A. Schoedel, M. Avramova, H. Utsuno, S. Bajorek, and A. Velazquez-Lozada. OECD/NRC BENCHMARK BASED ON NUPEC PWR SUBCHANNEL AND BUNDLE TESTS (PSBT). Technical report, US NRC and OECD Nuclear Energy Agency, 2010.

[31] P. Saha and N. Zuber. Point of Net Vapor Generation and Vapor Void Fraction in Subcooled Boiling. In Proceedings of the 5th International Heat Transfer Conference, volume 4, pages 175-179, 1974.

[32] R. Salko, M. Gergar, C. Gosdin, and M. Avramova. CTF Void Drift Validation. Technical Report CASL-U-2015-0320-002, Consrotium for Advanced Simulation of Light Water Reactors, 2015.

[33] R. Salko, M. Delchini, X. Zhao, D. Pointer, and W. Gurecky. Summary of CTF Accuracy and Fidelity Improvements in FY17. Technical Report CASL-U-2017-1428-000, Consortium for Advanced Simulation of Light Water Reactors, 2017.

[34] R. Salko et al. Ctf theory manual. Technical Report CASL-U-2019-1886-001, Consortium for Advanced Simulation of Light Water Reactors, 2019.

[35] R. Salko et al. Ctf validation and verification. Technical Report CASL-U-2019-1887-001, Oak Ridge National Laboratory, 2019.

[36] D. Steiner and J. Taborek. Flow boiling heat transfer in vertical tubes correlated by an asymptotic model. Heat transfer engineering, 13:43-69, 1992.

[37] Cajo JF Ter Braak. A markov chain monte carlo version of the genetic algorithm differential evolution: easy bayesian computing for real parameter spaces. Statistics and Computing, 16(3): 239-249, 2006.

[38] J.R.S. Thom, W.M. Walker, T.A. Fallon, and G.F.S. Reising. Boiling in subcooled water during flow up heated tubes for annuli. In Symposium on Boiling Heat Transfer in Steam Generating Units and Heat Exchangers, Manchester, London, 1965.

[39] Jasper A Vrugt, James M Hyman, Bruce A Robinson, Dave Higdon, Cajo J F Ter Braak, and Cees G H Diks. Accelerating markov chain monte carlo simulation by differential evolution with self-adaptive randomized subspace sampling. International Journal of Nonlinear Sciences and Numerical Simulation, 10(3), 12008.

[40] G.B. Wallis. One-Dimensional Two-Phase Flow. McGraw-Hill, 1969.

[41] D. Wells, P. Peturaud, and S. Yagnik. Overview of cfd round robin benchmark of the high fidelity fuel rod bundle nestor experimental data. In NURETH-16, Chicago, IL, August 30-September 4, 2015, 2015.

[42] Xu Wu, Tomasz Kozlowski, Hadi Meidani, and Koroush Shirvan. Inverse uncertainty quantification using the modular bayesian approach based on gaussian process, part 2: Application to trace. Nuclear Engineering and Design, 335:417 - 431, 2018. ISSN 0029-5493. doi: https://doi.org/10.1016/j.nucengdes.2018.06.003. URL http://www.sciencedirect.com/science/article/pii/S0029549318306411. 
[43] Jorgen Wurtz. An experimental and theoretical investigation of annular steam-water flow in tubes and annuli at 30 to 90 bar. Technical Report Riso Report No. 372, Riso National Laboratory, Copenhagen, Denmark, 41978.

[44] A. Wysocki and R. Salko. Validation of ctf droplet entrainment and annular/mist closure models using riso steam/water experiments. Technical Report CASL-U-2016-1080-000, Consortium for Advanced Simulation of Light Water Reactors, 2016.

[45] Xingang Zhao, Aaron J. Wysocki, Koroush Shirvan, and Robert K. Salko. Assessment of the subchannel code ctf for single- and two-phase flows. Nuclear Technology, 205(1-2):338-351, 2019. doi: 10.1080/00295450.2018.1507221. URL https://doi.org/10.1080/00295450.2018.1507221.

[46] N. Zuber and J. A. Findlay. Average volumetric concentration in two-phase flow systems. J. Heat Transfer, 87:453, 1965. 